\title{
VORTEX SHEDDING FROM A VIBRATING CABLE WITH ATTACHED SPHERICAL BODIES IN A LINEAR SHEAR FLOW;
}

\section{by}

Rodney Drew Peltzer;

Dissertation submitted to the Faculty of the Virginia Polytechnic Institute and State University in partial fulfillment of the requirement for the degree of DOCTOR OF PHILOSOPHY

in

Aerospace and Ocean Engineering

APPROVED :

\section{1.}

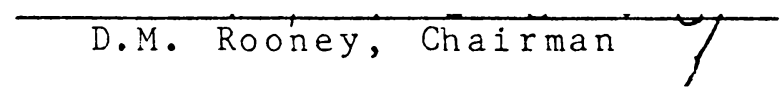

A

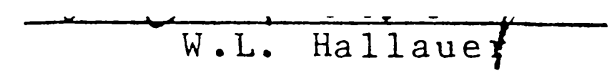

W.S. Saric

A.G. Szeless

August, 1982

Blacksburg, Virginia 


\section{ACKNOWL EDGEMENTS}

The present work was funded by the Naval Civil Engineering Laboratory in Port Hueneme, California (NRL Contract N00173-80C0211). of the Naval Research Laboratory in Washingt on, D.C. was the Technical Monitor.

The author wishes to thank for his comments and suggestions regarding the dissertation as it was being written. The ever present assistance and guidance of Dr. David M. Rooney, committee chairman, is greatly appreciated. The author wishes to thank the four other committee members; Dr. W.L. Hallauer, Dr. W.S. Saric, Dr. J.A. Schetz and Dr. A.G. Szeless, for their generosity in providing some of the instrumentation and ideas used toward the successful completion of this study.

The author would also like to express his graditude to the basement crew; for all the help and advice they provided to him during his four years with them in the basement of Randolph Ha11. 
Acknowledgments............................... ii

List of Figures............................... vi

Nomenclature.................................

I. INTRODUCTION. ............................... 1

I I. BACKGROUND AND LITERATURE.................... 5

A. Development of the Karma n Vortex St reet Wake..... 5

B Vortex Formation Length and Wake Width behind

St ationary Cylinders in Uniform Flow........... 9

C. Lock-on or Synchronization Phenomena: An overview of studies dealing with changes induced in the near wake of a rigid cylinder

by self-excited or forced motion of the body ....... 13

D. Lock-on or Synchronization Phenomena: An overview of studies dealing with changes induced in the near wake of a flexible cylinder or cable by self-excited or forced motion of the body.................... 20

E. Stationary and Vibrating Bluff Bodies in

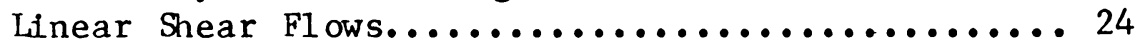

F. Vortex Shedding From a Sphere............... 30

G. Procedure Used to Design a Workable Experimental Test Setup.............................. 32

H. A Brief Discussion of the Fast Fourier Transform and Related Topics (Power Spectra)............. 34 
II . EXPER IMENTAL APPARATUS AND INSTRUMENTATION.......... 38

A. Wi nd Tunnel Test Facility................. 38

B. Test Mbde1.............................. 40

C. Experimental Test Setup................... 41

D. Cable Mode Shape Vibration Amplitude and Frequency Me asurement...................... 42

E. Vortex Shedding Frequency Measurement............ 44

F. Cable Wake Width and Vortex Formation Length Instrumentation

IV. EXPERTMENTAL METHODS....................... 46

A. Cable Mode Shape and Vibration Frequency Me a surement............................ 46

B. Cable Lock-on Region Tests.................. 47

C. Vortex Formation Length $\left(L_{f}\right)$ and Wake

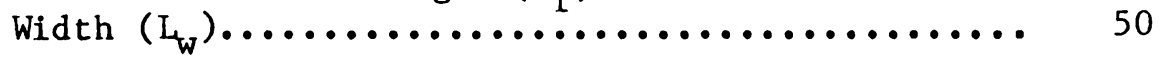

D. Sphere Lock-on Regions.................... 51

E. Sphere Region of Influence................ 53

F. Spanwise Sheared Flow Tests................. 55

V. RESULTS AND DISCUSSION....................... 59

A. Cable Mode Shapes........................ 59

B. Lock־on Phenomena........................ 60

C. Formation Lengt hs and Wake Widths............. 74

D. Sheared Flow Results....................... 79 
VI. $\operatorname{SUMMARY} \ldots \ldots \ldots \ldots \ldots \ldots \ldots \ldots \ldots \ldots \ldots \ldots \ldots \ldots \ldots \ldots \ldots . \ldots \ldots$

A. Findings and Conclusions................. 93

B. Recommendations for Further Study ............ 96

REFERENCES................................. 98

AP PENDIX A. FIGURES.......................... 106

APPEND IX B PREVIOUSLY ESTABLISHED METHODS.............. 157

viTA...................................... 161

ABSTRACT 
1. Vortex Formation Lengths for Circular Cylinders in Uniform and Linear Shear Flows.............. 107

2. Wake Widths for Circular Cylinders in Uniform

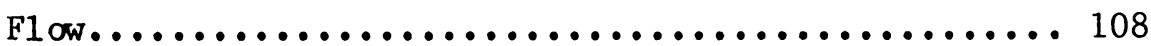

3. Wind Tunnel Test Section and Cable Model

(Top View).............................. 109

4. Wind Tunnel Test Section and Cable Model (Downstream View)......................... 110

5. Spanwise Velocity and Turbulence Profiles

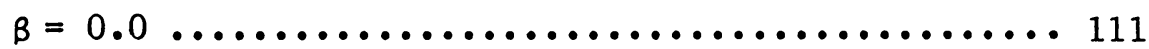

6. Spanwise Velocity and Turbulence Profiles

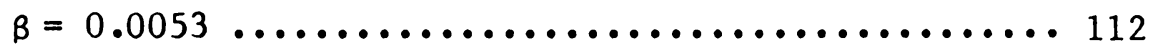

7. Cable Tension Me asurement........................ 113

8. Forced Cable Vibration....................... 114 
9. Cable Mode Shape, Vibration Amplitude

and Frequency Measurement.......................... 115

10. Vortex Shedding Frequencies Measurement........... 116

11. Cable Wake Width and Vortex Formation

Length Instrumentation................... 117

12. Cable Mode Shape (First Mo de) .................. 118

13. Cable Mode Shape (Second Mode).................. 119

14. Cable Lock-on Regions as a Function

of Vibration Amplitude..................... 120

15. Reduced Velocity at the Boundaries of the Lock-on Region for the Vibrating

Cable as Function of the Vibration Amplitude...... 121

16. Variation of the Strouhal Number as a Function of Vibration Amplitude at the Boundaries of the Lock-on Region.......................... 122 
17. Variation of the Centerline Strouhal Number as a Function of Reynolds Number of a Stationary and Vibrating Cable.

18. Spanwise Behavi or of the Vortex Shedding

Frequency during Lock-on, $\operatorname{Re}_{c}=3.83 \times 10^{3}$,

Second Mode Shape, $a / d=11.7 \%, \beta=0.0 \ldots \ldots \ldots . . .124$

19. Spanwise Variation of the Vortex Shedding Frequency below the Lock-on Range, $\beta=0.0 \ldots \ldots . .125$

20. Spanwise Variation of the Vortex Shedding Frequency away from the Lock-on Region, First Mode, $\beta=0.0 \ldots \ldots \ldots \ldots \ldots \ldots \ldots \ldots \ldots \ldots \ldots$

21. Spanwise Variation of the Vortex Shedding Frequency away from the Lock-on Range, First Mode, $\beta=0.0$

22. Sphere Lock-on Regions as a Function of Vibration Amplitude ${ }^{a} / d_{s} \ldots \ldots \ldots \ldots \ldots \ldots \ldots \ldots \ldots . \ldots 128$ 
23. Reduced Velocity at the Boundaries of the Lock-on Region for the Vibrating Sphere as a Function of the Vibration Amplitude.......... 129

24. Spanwise Strouhal Number Variation Along the Vibrating Cable with Five Spheres in Unsheared $\mathrm{F} 1 \mathrm{ow}, \mathrm{a} / \mathrm{d}=24.2 \%$, First Mode, $\operatorname{Re}_{c}=2.23 \times 10^{3}$, Away from Lock-on Range....... 130

25. Ty pical Formation Length and Wake Width Traces..... 131

26. Variation of $L_{f}$ and $L_{w}$ as a Function of Increasing Reynolds Number. .................. 132

27. Variation of $L_{f}$ and $L_{W}$ as a Function of Increasing Reynolds Number. .................. 133

28. Variation of $\mathrm{L}_{\mathrm{f}}$ and $\mathrm{L}_{\mathrm{W}}$ as a Function of Increasing Reynolds Number. ................... 134

29. Variation of $L_{f}$ and $L_{w}$ as a Function of Increasing Reynolds Number. ................. 135 
30. Variation of $\mathrm{L}_{\mathrm{f}}$ and $\mathrm{L}_{\mathrm{W}}$ as a Function of Increasing Reynolds Number................... 136

31. Power Spectra Showing the Spanwise Variation of the Vortex Shedding Frequency, First Phase Test, $\operatorname{Re}_{c}=2.6 \times 10^{3}$, No Vibration, No Spheres, $\beta=0.0053 \ldots \ldots \ldots \ldots \ldots \ldots \ldots \ldots \ldots \ldots \ldots . . \ldots 137$

32. Spanwise Strouhal Number Variation, First Phase Test; $\operatorname{Re}_{c}=2.6 \times 10^{3}$, No Vibration, No Spheres, $\beta=0.0053 \ldots \ldots \ldots \ldots \ldots \ldots \ldots \ldots \ldots \ldots \ldots 138$

33. Power Spectra Showing the Spanwise Variation of the Vortex Shedding Frequency, First Phase Test, $\operatorname{Re}_{c}=2.6 \times 10^{3}$, First Mode Vibration, $a / d=23.3 \%$, No Spheres, $\beta=0.0053 \ldots \ldots \ldots \ldots \ldots . .139$

34. Spanwise Strouhal Number Variation, First Phase Test, $\operatorname{Re}_{c}=2.6 \times 10^{3}$, First Mode Vibration, $a / d=23.3 \%$, No Spheres, $\beta=0.0053 \ldots \ldots \ldots \ldots \ldots . . .140$ 
35. Power Spectra Showing the Spanwise Variation of the Vortex Shedding Frequency, First Phase Test, $\operatorname{Re}_{c}=3.72 \times 10^{3}$, No Vibration, No Spheres, $\beta=0.0053 \ldots \ldots \ldots \ldots \ldots \ldots \ldots \ldots \ldots \ldots \ldots 141$

36. Spanwise Strouhal Number Variation, First Phase Test, $\operatorname{Re}_{c}=3.72 \times 10^{3}$, No Vibration, No Spheres, $\beta=0.0053 \ldots \ldots \ldots \ldots \ldots \ldots \ldots \ldots \ldots \ldots . \ldots 142$

37. Power Spectra Showing the Spanwise Variation of the Vortex Shedding Frequency, First Phase Test, $\operatorname{Re}_{c}=3.72 \times 10^{3}$, Second Mode Vibration, $\mathrm{a} / \mathrm{d}=11.7 \%$, No Spheres,

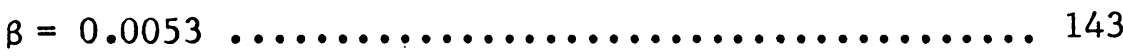

38. Spanwise Strouhal Number Variation, First Phase Test, $\operatorname{Re}_{c}=3.72 \times 10^{3}$, Second Mode Vibration, $a / d=11.7 \%$, No Spheres, $\beta=0.0053 \ldots \ldots \ldots \ldots \ldots \ldots \ldots \ldots \ldots \ldots \ldots \ldots \ldots \ldots$ 
39. Power Spectra Showing the Spanwise Variation

of the Vortex Shedding Frequency,

$\operatorname{Re}_{c}=2.96 \times 10^{3}$, No Vibration,

No Spheres, $\beta=0.0053$

145

40. Spanwise Strouhal Number Variation,

$\operatorname{Re}_{c}=2.96 \times 10^{3}$, No Vibration,

No Spheres, $\beta=0.0053 \ldots \ldots \ldots \ldots \ldots \ldots \ldots \ldots \ldots$

41. Power Spectra Showing the Spanwise Variation

of the Vortex Shedding Frequency,

$\operatorname{Re}_{c}=2.96 \times 10^{3}$, First Mode Vibration,

$a / d=29.1 \%$, No Spheres, $\beta=0.0053$

42. Spanwise Strouhal Number Variation,

$\operatorname{Re}_{c}=2.96 \times 10^{3}$, First Mode Vibration,

$a / d=29.1 \%$, No Spheres, $\beta=0.0053$

43. Power Spectra Showing the Spanwise Variation

of the Vortex Shedding Frequency,

$\operatorname{Re}_{c}=2.96 \times 10^{3}$, Five Spheres,

No Vibration, $\beta=0.0053$

149 
Page

44. Spanwise Strouhal Number Variation,

$\operatorname{Re}_{c}=2.96 \times 10^{3}$, Five Spheres,

No Vibration, $B=0.0053$

150

45. Power Spectra Showing the Spanwise Variation

of the Vortex Shedding Frequency,

$\operatorname{Re}_{c}=2.96 \times 10^{3}$, Five Spheres, First

Mo de Vibration, $a / d=23.3 \%, \quad \beta=0.0053 \ldots \ldots . . .151$

46. Spanwise Strouhal Number Variation, $\operatorname{Re}_{c}=2.96 \times 10^{3}$, Five Spheres, First

Mode Vibration, $a / d=23.3 \%, \quad \beta=0.0053 \ldots \ldots \ldots . .152$

47. Power Spectra Showing the Spanwise Variation

of the Vortex Shedding Frequency,

$\operatorname{Re}_{c}=2.96 \times 10^{3}$, Three Spheres,

No Vibration, $\beta=0.0053$

48. Spanwise Strouhal Number Variation,

$\operatorname{Re}_{c}=2.96 \times 10^{3}$, Three Spheres,

No Vibration, $\beta=0.0053 \ldots \ldots \ldots \ldots \ldots \ldots \ldots \ldots \ldots . \ldots . \ldots 154$ 
Page

49. Power Spectra Showing the Spanwise Variation

of the Vortex Shedding Frequency,

$\operatorname{Re}_{c}=2.93 \times 10^{3}$, Three Spheres, First

Mo de Vibration, $a / d=23.5 \%, \beta=0.0053 \ldots \ldots \ldots .155$

50. Spanwise Strouhal Number Variation,

$\operatorname{Re}_{c}=2.93 \times 10^{3}$, Three Spheres, First

Mode Vibration, $a / d=23.5 \%, \quad \beta=0.0053 \ldots \ldots \ldots . .156$ 


\section{Nome nclature}

a/a $\max$ nondimensional mode shape amplitude

a/d local nondimensional vibration amplitude

$a_{\max } / d$ nondimensional vibration amplitude at an antinode

$\mathrm{C}_{\mathrm{D}} \quad$ drag coefficient

$\mathrm{C}_{\mathrm{pb}} \quad$ base pressure coefficient

d general bluff body diameter (cable, cylinder, etc.)

$\mathrm{d}_{\text {s }} \quad$ sphere diameter

$\mathrm{f}_{\mathrm{c}} \quad$ Nyquist folding f requency

$\mathrm{f}_{\text {cso }} \quad$ vortex shedding frequency from a cable

$\mathrm{f}_{\mathrm{csv}} \quad$ vortex shedding frequency from a vibrating cable

$\mathrm{f}_{\mathrm{cv}} \quad$ forced cable vibration frequency 


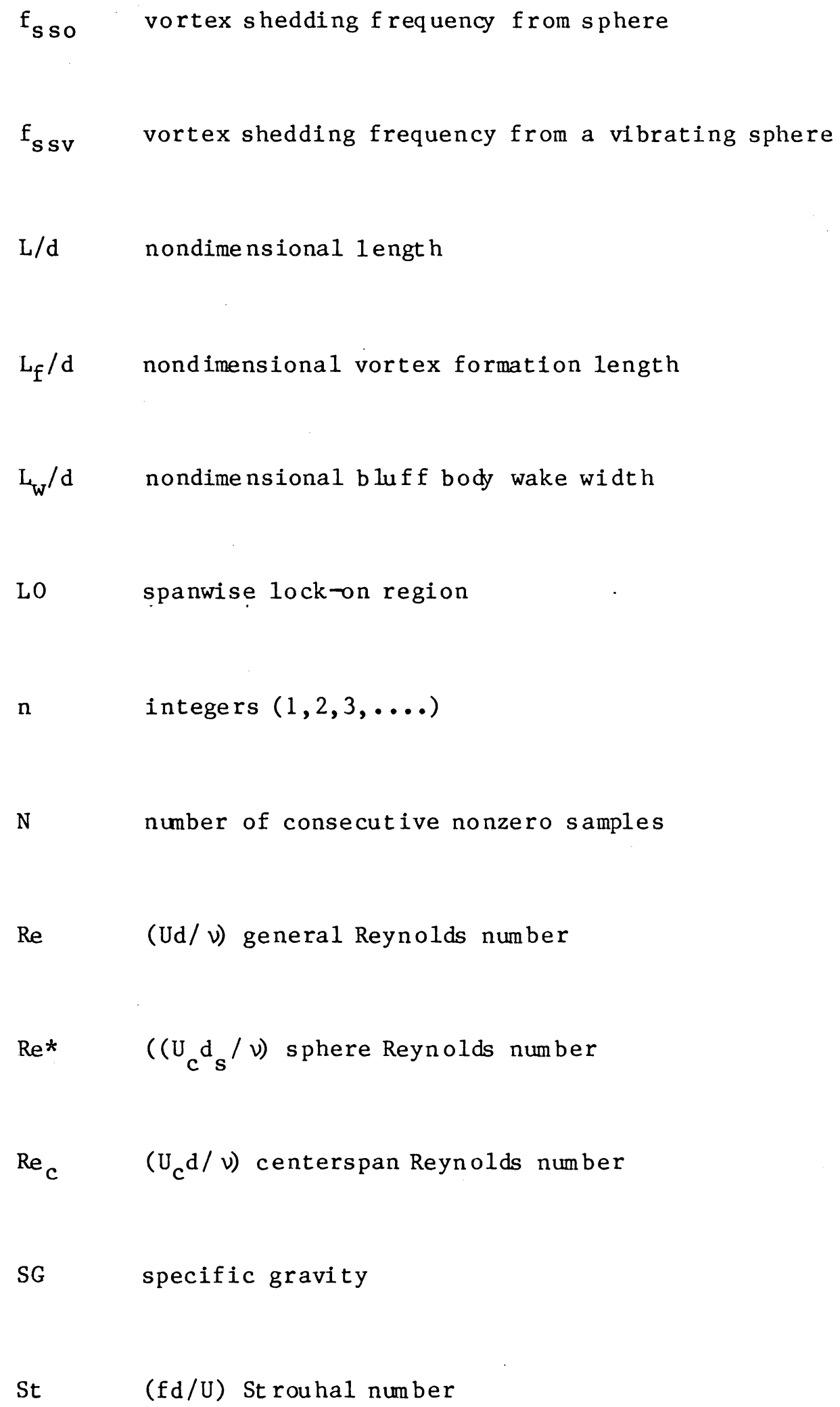


St $\quad\left(f_{c s v} d / U_{c}\right)$ centerspan St rouhal number

$\mathrm{St}^{*} \quad\left(\mathrm{f}_{\mathrm{sso}} \mathrm{d}_{\mathrm{s}} / \mathrm{U}_{\mathrm{C}}\right)$ sphere Strouhal number

$S_{\text {csv }} \quad$ St rouhal number from a vibrating cable

$\mathrm{S}_{\mathrm{cv}} \quad$ Strouhal number of the cable vibration

$t \quad$ time

$\mathrm{T} \quad$ cable tension $\left(1 \mathrm{~b}_{\mathrm{f}}\right)$

$\mathrm{T}_{\mathrm{r}} \quad$ data record

Tu turbulence intensity

U velocity (loca1)

$\mathrm{U}_{\mathrm{C}} \quad$ velocity at wind tunnel centerspan

$U_{r} \quad\left(U_{c} / f_{c v} d\right)$ reduced velocity

$U_{r}^{*} \quad$ critical reduced velocity

$x(t) \quad$ analog signal 
$\mathrm{x} / \mathrm{d} \quad$ nondimensional vertical location

y/d nondimensional spanwise (horizonta1) location

z/d nondimi nensional streamwise (d ownstream) location

$\beta \quad\left(d / U_{c} d U / d y\right)$ shear parameter

$\Delta \quad$ spectral resolution bandwidth

$\Delta \quad$ sampling frequency (FFT)

$v \quad$ kinematic viscosity

$\rho \quad$ mass density $\left(1 b_{f} \sec ^{2} / f t^{4}\right)$

$\omega_{n} \quad$ natural frequency of vibration 


\section{Int roduction}

Flexible cylindrically shaped bodies are often an integral part of a much larger, highly complex structural system such as an offshore drilling platform, which has mooring systems and drilling risers that are flexible and cylindrical. They are also part of cable networks used to support marker buoys, delicate instrument arrays, and towed cable arrays. Vortices are shed as the surrounding fluid separates alternately from opposite sides of the high aspect ratio, bluff cylindrical body when it is towed, or when waves and currents flow past it. This periodic vortex shedding is responsible for producing steady and unsteady drag forces in the direction of the flow and unsteady lift forces in the cross flow direction. When these vortices are shed at a frequency close to one of the natural frequencies (or a multiple thereof) of the structural system, the shedding can lock-on to (synchronize with) the natural frequency. If the damping is sufficiently small, intensified large amplitude cross flow oscillations, increased stresses, amplified acoustic flow noise, early fatigue or shorter failure life, increased hydrodynamic forces, and the chance of global structural or instrument damage will occur. This particular problem, encountered when designing the structural system, is called cable-struming and is not we 11 understood. Further complicating the design procedure is the vertically sheared current profile that is typically found in the oceans. The vortex shedding frequency along the bluff body span can vary with depth 
in this case. Synchronization and its related phenomena may occur over part of the total bluff body span.

To have a reasonable amount of confidence in the design and safety of the structural system, the designer must have some understanding of the dynamic behavior of the system. Numerous references to vortex shedding-related structural problems in the literature attest to the fact that substantial research into vortex related synchronization phenomena must be performed and analyzed. The following are a few examples of the problems associated with full scale systems that were not designed properly to suppress or eliminate vortex shedding, and some of the attempted solutions to these problems.

Severe oscillations of piles and pile-supported structures, caused by vortex shedding, occurred in a tidal flow during the construction of a deep water oil terminal at Immingh am the Humber estuary in England. Extensive on-site investigations into the phenomenon permitted the terminal to be redesigned and completed safely. As a result of this near disaster, a three year, full-scale investigation into the oscillations of piles in marine structures was undertaken at Imminghan, and the results were published by Wootton, Warner, Sainsbury, and Cooper [49].

Over a two and one half year period, each of eight single pile aids-to-navigation structures in the San Pablo Bay of California vibrated to failure at least once. Vortex shedding from the piles in the six knot current caused them to vibrate violently and to fail. A 
helical strake kit was employed by the U.S. Coast Guard in an attempt to alleviate this problem. In a report describing the effectiveness of the helical strake kit, walker [47] concluded that the kit eliminated the vortex shedding and stabilized the structure over a wide range of flow speeds. The ninth single pile aid-to-navigation has had no vortex shedding related problems to this date.

The deep water piling installations and pile driving operations for the COGNAC platform were thought to be susceptible to vortex shedding related problems. Every, King and Griffin [11] and Fischer, Jones and King [12] have studied the flow-induced vibration of a cable-suspended pile and vortex induced motions of a cantilever pile, respectively, in an effort to predict and develop methods to reduce or eliminate the vibrations and vibration related phenomena. Both groups of authors used full scale and scaled model tests in their experime ntal analy ses.

The present study was undertaken to examine the behavior of a flexible, high aspect ratio $(\mathrm{L} / \mathrm{d}=107)$ marine cable in a linear shear flow. Partialar attention was paid to lod-on or "synchronization" phenomena associated with uniform and sheared flow past the cable when it is forced to vibrate in the first mode. The study was extended to include an analysis of the effects on the vortex shedding and synchronization phenomena that are generated by placing distributions of spherical bluff body shapes along the span of the cable in uniform and sheared flow. The results discussed in this paper will then be used in conjunction with recent experimental 
and analytical studies to develop the capability to predict the strumming response of marine cables in a variety of different flow situations.

The most recent of these field experiments was conducted in the mouth of Holbrook Cove near Castine, Maine during the summer of 1981 to study the strumming vibrations of marine cables. One of the objectives of those experiments was to validate and, if necessary, to provide a data base for modifying the computer code NATFREQ which was developed by the Naval Civil Engineering Laboratory for calculating the natural frequencies and mode shapes of taut cables with large numbers of attached discrete masses. In a recent paper, Vandiver and Griffin [46] describe the field test progran and present some initial results from it. They compare calculations using the NATFREQ code with selected test data that have been analyzed in sufficient detail. Time histories of the measured hydrodynamic drag coefficients, current speeds, and cable strumming responses are presented and discussed there. 
II. Ba ckg round and Literature

A. Development of the Karman Vortex Street Wake

Before discussing any previous theoretical and experimental studies dealing with fluid dynamic properties in the wakes of stationary and vibrating cylinders and cables in a uniform stream, a detailed summary of the development of the Karman vortex street, from Reynolds numbers of unity (Stokes flow) through the end of subcritical flow, is presented. Throughout this discussion, it is assumed that the turbulence leve 1 is sma $11 \underline{\text { i.ee, }}$ less than 1 percent.

(1) Stokes flow, $0<\operatorname{Re}<$ 5: For Reynolds numbers of order unity, the flow around the body behaves as if it were inviscid. The laminar boundary layer separates at the rear stagnation point. The flow along the cylinder span is strongly two-dimensional. As the Reynolds number approaches five, the laminar boundary layer begins to separate before the rear stagnation point. There are no vortices present in this Stokes flow region.

(2) Symmetrical Range, $5<\operatorname{Re}<40$ : This range is sometimes referred to as "Föpp1 Flow". A captive vortex pair (F̈ppl Vortices) appears in the wake of the cylinder. The separation point has moved further away from the rear stagnation point, towards \pm 90 degrees. The boundary layer remains laminar after separation and the flow is still strongly two-dimensiona1. The shear layer (i.e., the interaction between the separated main flow and stagnated flow region) supplies vorticity to this captured vortex pair, causing them 
to acquire more strength and grow as the Reynolds number approaches 40. They remain attached to the cylinder.

(3) Lami nar "Regular" Vortex Shedding Region, $40<\operatorname{Re}<125$ to 150: For $\operatorname{Re}=40$ to 80 , the vortices seem to form from a wake instability over several diameters downstream. After $\operatorname{Re}=80$ or 90 , when the vortex grows strong enough on one side, it begins to roll up and draw the opposite shear layer across the wake. The vorticity of opposite sign permits no more vorticity input into the growing vortex. The vortex is then shed and travels downstream. Due to some flow or wake instability or asymmetry, or surface irregularity, the shedding process is asymmetric, i.e. the first vortex is shed from one side of the cylinder, and the second is shed a short time later, while a new vortex is forming on the opposite side to replace the one already shed. These alternating vortices which are shed into the wake are called vortex streets. They were first theorized by von Kármán. These entirely laminar vortices slowly decay through viscous dissipation and can be measured clearly as far as 100 diameters downstream. The flow is again two-dimensional. Schaefer and Eskinazi [43] and Griffin [15] divide the laminar wake into three regions. First is the formation region, where the vortex street develops from the interaction of the shear layers. The formation region ends where the first periodic vortices are shed. Next is the stable region, where the vortices in the fully developed laminar vortex street exhibit a regular periodicity. The transverse vortex spacing increases and the vortex cores spread by viscous 
action. The amplitude of the vortex velocity fluctuations diminishes. The last is the unstable region, where the vortex street exhibits an irregular behavior and eventual turbulent breakd own. There is a decrease in the transverse spacing of the vortices. At greater downstream distances there is a reappearance of the vortex street with different geometric properties. The Strouhal number is related to the Reynolds number in this region by Roshko's empirical formula, St $=0.212-4.49 /$ Re.

(4) Lami nar Turbulent Transition, 125 to $150<\operatorname{Re}<300$ to 350 : In this region the flow becomes three-dimensional. The wake is characterized by an intermittent type of flow downstream of the formation region, where the laminar vortices deteriorate, through low frequency modulation and irregularities, to turbulent vortices. As the Reynolds number increases, more and more vortices become turbulent, until the entire downstream shedding process is turbulent. The distribution of spectral energy under the vortex shedding peak decreases as more energy is transferred to turbulence. The vortex lines are no longer straight and para1le1 to the cylinder axis, but become inclined to 1 t.

(5) Turbulent Vortex Range, 300 to $400<\operatorname{Re}<2.0$ to 4.0 $\times 10^{5}:$ The Strouhal number $\left(\mathrm{St}=\mathrm{f}_{\mathrm{cso}} \mathrm{d} / \mathrm{U}_{\mathrm{c}}\right)$ is a constant throughout this range at a value around 0.20 . The boundary layer is still laminar and separates from the cylinder approximately 82 degrees from the forward stagnation point. The transition to turbulence ocars in the separated layers before the vortices are 
formed (i.e. in the formation region). The turbulent vortices rapidy diffuse in the downstream direction, becoming part of the turbulent wake. This large region can be further divided into three subregions [7].

(5.1) Lower Turbulent Vortex Region, $400<\operatorname{Re}<1.3 \times 10^{3}$ : The position signifying the beginning of the transition to turbulence is close to the end of the vortex formation region The region of transition is about three diameters long and includes the end of the formation region. The development of turbulence begins with the observation of small turbulent bursts before the end of the formation region. These bursts become more frequent further downstream of the formation region in the wake. At the point where the fully developed periodic wake begins, every vortex consists of turbulent fluid. In the immediate downstream vicinity of the formation region, the vortex sheet becomes fully turbulent.

(5.2) Intermediate Turbulent Vortex Range, $1.3 \times 10^{3}<$ Re $<8.0$ $x 10^{3}$ complete transition of the shear layers to turbulence takes place in the vortex formation region. The onset of turbulence occurs in the following manner. Directly downstream of the cylinder, the separated shear layers are lami nar and exhibit a domi nant fundamental frequency. Still further downstream in the formation region, the first turbulent bursts, which are waves at a single higher frequency, are imposed on the fundamental frequency. Further downstream in the formation region the fundamental frequency is masked by the higher frequency, and the flow degenerates to turbulence. The periodic wake 
with turbulent vortices is only present in this region and beyond. As the Reynolds number increases, the transition point moves from 1.2 to 0.7 diameters behind the center axis of the cylinder.

(5.3) Upper Turbulent Vortex Region, $8.0 \times 10^{3}<\operatorname{Re}<2.0$ to 4. $0 \times 10^{5}$ : Laminar periodic flow is observed inside the separated boundary layer at the shoulder of the cylinder. This flow completely degenerates to turbulence in less than one-half a diameter downstream of the cylinder axis. There is then a period of complete turbulent motion until the periodic wake is formed (at the end of the formation region).

Briefly summarizing, transition of the vortices to turbulence occurs in two ways. When the vortices are laminar upon formation, distortions by large scale three-dimensionalities in the flow cause the vortices to become turbulent downstream. When the vortices are turbulent upon formation, some amplification of a two-dimensional instability produces transition waves which eventually lead to turbulence through small scale three-dimensionalities. The transition takes place in the separated layers before they roll into vortices.

B. Vortex Formation Length and Wake Width Behind Stationary Cylinders in Uniform Flow

The vortex formation length, $L_{f}$, is a measure of the downstream extent of the vortex formation region in the cylinder wake. The vortex formation length is generally defined as the distance between 
the cylinder center axis and the first downstream appearance of the fully formed periodic vortex street. There are four different criteria that define how $L_{f}$ can be measured [34]. They are summa rized:

(1) The rms maximum of the second harmonic of the fluctuating velocity on the axis of the wake.

(2) The minimum of low frequency modulation of the vortex signa1.

(3) The minimum of the mean pressure on the wake axis.

(4) The minimum transverse spacing from the wake axis of the peaks of the maximu fluctuating velocity.

The first criterion was used in this study, as we 11 as in most previous studies, e.g., by Bloor and Gerrard [8], Griffin [15], Ramberg [34], Peltzer and Rooney [31], and Woo et al [48].

There are three experimental studies dealing with the measurement of the formation length in the Reynolds number range covered by the present study. Figure (1) shows a summary of the results obtained by Bloor and Gerrard [8], Woo et al [48], and Peltzer. These results span the Reynolds number range $8.0 \times 10^{2}$ to $1.5 \times 10^{5}$. Bloor and Gerrard measured $\mathrm{L}_{\mathrm{f}}$ behind five cylinders of aspect ratio $\mathrm{L} / \mathrm{d}=20,40,80,160$, and 230 in their 20 in. $x 20$ in. test section. The majority of their measurements were taken in the wakes of smooth brass cylinders of aspect ratio 20 and 160 . Their results show a dependence of $\mathrm{L}_{f}$ on cylinder diameter. The curves shift over to the right with increasing cylinder diameter (decreasing 
aspect ratio), a phenomenon that the present author believes can be related to the interaction between end effects generated by the tunnel walls and decreasing aspect ratio. Bloor and Gerrard's cylinders spanned the tunnel vertically and had no end plates. During the present set of tests, the author observed that end effects lengthen the vortex formation region and stabilize the shedding pattern along the cylinder span. It is suggested here that as the aspect ratio decreases, end effects have a predominant influence on the vortex formation length and sparwise vortex strength and stability. This observation partially explains why Bloor and Gerrard's results are dependent on cylinder diameter, or decreasing aspect ratio, and end effect interactions. Another factor contributing to the scatter in their data was their observation in a later paper that the flow was not uniform across the wind tunnel span [13]. Only the central 7.5 inches experienced a uniform flow. This nonuniformity was probably due to the combination of test section design and tunnel wa 11 effects.

The formation length data of Woo et al and Peltzer fall onto the solid curve. There is not much scatter in the data presented by both authors as they both were careful to eliminate (or minimize) end effects. Woo et al measured $L_{f}$ at the centers of five very hard, very smooth, brass tubes of aspect ratios $\mathrm{L} / \mathrm{d}=16,24,36,48$, and 96 in various 1 ow and moderate linearly sheared flows. Both Peltzer and Rooney [31] and Woo et a1 have shown that the formation length values are not significantly affected by 1 ow and moderate linearly 
sheared flows. Peltzer measured $\mathrm{L}_{\mathrm{f}}$ behind two machined smooth aluminum cylinders of aspect ratios $\mathrm{L} / \mathrm{d}=17.3$ and 27 . At a Reynolds number $1.0 \times 10^{3}$, the formation lengt $\mathrm{h}, \mathrm{L}_{\mathrm{f}} / \mathrm{d}$ is 2.8 . As the Reynolds number increases, $\mathrm{L}_{\mathrm{f}} / \mathrm{d}$ decreases until it becomes constant at a value $\mathrm{L}_{\mathrm{f}} / \mathrm{d}=1.32$ when the Reynolds number reaches $1.0 \times 10^{4}$. The formation length then remains constant until $\operatorname{Re}=1.6 \times 10^{5}$.

To the best of the author's knowledge, no experimental studies have been published dealing with the formation lengths or wake properties behind helically wound cables. Since it is known that roughness stabilizes the vortex shedding structure behind a circular cylinder [32] by lengthening the vortex formation region, it is postulated that the helical shape will lengthen the vortex formation region when comared to that region measured behind a circular cylinder at the same Reynolds number.

Once the vortex formation length has been determined, the wake width, $L_{W}$, can be measured at that point. The most recent compehensive summary of the variation of wake widths behind circular cylinders as a function of Reynolds number is presented by Griffin [19]. A copy of his plot of $L_{w}$ vs. Re for the Reynolds number range $10^{2}$ to $2.0 \times 10^{5}$ is shown in Figure 2, along with the recent experimental data of Peltzer. Only four experimentally measured values of $\mathrm{L}_{\mathrm{w}} / \mathrm{d}$ existed in Griffin's data range between Reynolds numbers of $3.0 \times 10^{3}$ to $2.0 \times 10^{5}$. Griffin determined the values of $\mathrm{L}_{\mathrm{w}} / \mathrm{d}$ that defined the cross hatched region, between the Reynolds numbers $3.0 \times 10^{3}$ and $2.0 \times 10^{5}$, using a relation derived from 
Roshko's free streamline theory. This relation assumes that the base pressure is constant across the wake and that there are no roughness effects on transition or separation bubbles. The relation is $\mathrm{L}_{\mathrm{w}} / \mathrm{d}$ $=\left(C_{D} /\left(-C_{p b}\right)\right)$. The relation is valid throughout the range in question since the base pressure generally remains uniform across the cylinder base region and all of the cylinders were smooth. Peltzer's values lie between $\mathrm{L}_{\mathrm{w}} / \mathrm{d}=0.94$ and 0.99 , falling directly below Griffin's cross hatched range. Contained within and defining Peltzer's cross hatched range were 20 data points, which were measured at equally spaced Reynolds number intervals. It should be noted that Griffin only had one measured data point in the Reynolds number range covered by Peltzer's data.

C. Lock-on or Synchronization Phenomena: An overview of studies dealing with changes induced in the near wake of a rigid cylinder by self-excited or forced motion of the body.

When a bluff body (rigid or flexible cylinder or flexible cable) is immersed in a uniform stream or spanwise sheared flow, a Karman vortex street is generated in the wake of the body for Reynolds numbers between 50 (pure laminar vortices) and 2.0 to $4.0 \times 10^{5}$ (turbulent vortices). When the vortex shedding frequency approaches a natural frequency (or submultiple thereof) of the structure, resonant flow-induced oscillations of the cable, bluff body, or flexible cylinder may occur if the damping of the system is small enough. When the shedding frequency gets close enough to the natural 
frequency, the body captures the shedding frequency in an apparent violation of the Strouhal relationship. The shedding frequency and body oscillations then synchronize at a frequency close to the natural frequency of that body. These self-excited oscillations are usually normal to the incident flow direction and in water may be as large as two to four diameters in peak to peak amplitude. In line oscillations sometimes ocar in water where the hydrodynamic forces are considerably larger than in air. The vortex shedding frequency is captured by the natural frequency over a range of flow speeds, the start of which occurs at a flow speed which corresponds to the natural vibration frequency. During this lock-on, there are changes induced in the near wake of the body by the self-excited resonant motion. There are many different terms used to characterize these phenomena of which sychronization, cable strumming, lock-on, wake capture, self-induced or self-excited oscillations, are the most common.

It was determined that the cable system to be studied in the present tests was too highly damped to be self-excited in an air flow. Some type of forced excitation would then be necessary to simulate the self-excited resonant conditions in order to study the lock-on induced changes in the cable wake. The question that must be addressed now is whether self-excited resonant flow-induced changes in the near wake can be reproduced with an externally excited arrangement. Griffin [16] has addressed and answered this question. His study was directed towards analyzing the similarity in 
the periodic wakes of two oscillating cylinders in the Reynolds number range 550 to 900 , one of which was forced to vibrate sinusoidally normal to the flow, while the other was tuned to self excite under the influence of fluid forces. When the near wakes behind the free and forced vibrating cylinders were comared, the near wake flow conditions and phase relations were found to be similar when the experimental conditions were carefully duplicated. Changes in the wake that accompany resonant vortex-excited oscillations are reproducible from forced externally excited oscillations. This method also has the advantage that the amplitude of vibration, and the natural frequency and mode shape, can be varied independently. The behavior of the lock-on phenomena when the mode shape, vibration amplitude, and natural frequency are varied can be studied over a wide range of Reynolds numbers that is not exclusively limited to the lock-on range associated with self-excited flowinduced vibrations.

Koopmann [25] performed one of the earliest experimental studies directed toward analyzing what effects the forced vibration of a circular cylinder in uniform fluid flow has on the resultant near wake properties at Reynolds numbers less than 300. The frequency range $\left(\mathrm{f}_{\mathrm{csv}} / \mathrm{f}_{\mathrm{cv}}\right)$ over which lock-on occurred increased as the amplitude of cylinder vibration, a/d, increased. The slantwise vortex shedding pattern exhibited in flow behind the stationary cylinder was replaced by parallel vortex shedding (parallel to the cylinder centeraxis). His flow visualization photographs showed that 
the correlation length, defined as the spanwise distance along the cylinder span over which the vortex shedding is in phase, increased rapidly as a/d increased. This increase has since been found to be much greater when the vortices are laminar upon formation [15, 41], than when they had become turbulent ( $\operatorname{Re}>450)$. The level of freestream turbulence will also influence the amount that the correlation length increases during lodk-on [41]. For 1ow turbulence flows, the correlation length is estimated by extrapolation to Increase from $3.5 \mathrm{~d}$ to $40 \mathrm{~d}$, and when the flow is turbulent, it increases from $2.5 \mathrm{~d}$ to $10 \mathrm{~d}[41]$.

Griffin [15,16], and Griffin and Votaw [23] studied changes in the near wake of a forced vibrating cylinder for Reynolds numbers between 120 and 900. They found that significant changes in the near wake development were caused by the synchronized cylinder oscillations. The purely laminar vortex shedding range (which usually ends at $\operatorname{Re}=125$ to 150) was extended to a Reynolds number of 350. The transverse synchronized vibrations suppressed the initiation of turbulence downstream of the vortex formation region. They investigated the dependence of the general near wake structure (vortex formation length, vortex strength, and wake width) on vibration amplitude a/d and cylinder vibration frequency $\left(f_{c v} / f_{c s v}\right)$. The length of the vortex formation region was influenced by changes in both $\mathrm{a} / \mathrm{d}$ and $\mathrm{f}_{\mathrm{cv}} / \mathrm{f}_{\mathrm{csv}}$, when the cylinder and vortex shedding frequencies were locked-on. The formation length decreased uniformly as a/d was increased. $L_{f}$ decreased by as much as 59 
percent when comared to the non-vibrating case. $L_{f}$ also decreased as the ratio $\mathrm{f}_{\mathrm{cv}} / \mathrm{f}_{\mathrm{csv}}$ was increased from less-than to greater-than one. The wake width was found to increase with the amplitude of synchronized oscillations.

In two later studies, Griffin and Ramberg [21,22] measured the changes in vortex strength and spacing that were produced by varying the amplitude and cylinder vibration frequency in the synchronization range at a Reynolds number of 144. When a/d was increased, the vortex strength and vorticity generation were increased, and the lateral vortex spacing in the stable region of the wake decreased. The formation length again varied inversely with vibration frequency $\left(f_{c v} / f_{c s v}\right)$. The unstable region in the wake moved upstream as the ratio $\mathrm{f}_{\mathrm{cv}} / \mathrm{f}_{\mathrm{csv}}$ was increased, however the lateral spacing of the vortices in the stable region was relatively unaffected by the changes in $\mathrm{f}_{\mathrm{cv}} / \mathrm{f}_{\mathrm{csv}}$.

Davies [10] examined the turbulent near wakes of a stationary and forced oscillating D-shaped cylinder in the Reynolds number range $7.0 \times 10^{3}$ to $4.0 \times 10^{4}$. The cylinder vibration was synchronized with the vortex shedding frequency. He found,that there was a 35 percent increase in the strength of the vortices shed from the oscillating cylinder, and that the shedding was much more regular. He attributed this increase in regularity to the increased vortex strength. The increased vortex strength was the result of less destruction of vorticity as the shear layers interacted across the wake, or the shedding of more vorticity into the shear layers. Davies noted that 
the growing vortex seemed to roll up more quidkly (i.e. the formation length decreased). Zdravkovich [50] summarizes three major reasons why the vortex shedding behind a synchronized oscillating cylinder is stronger and more regular than that found behind a stationary one:

(1) The formation length decreases and the fluctuating and time averaged forces are magnified.

(2) The spanwise correlation of the vortex formation and shedding are enhanced remarkably by the cyclic oscillations.

(3) Since the synchronization frequency remains constant over a range of flow velocities, there is a constant period of time available for the formation. More vorticity is generated with increased velocity within and along the synchronization range and the vortices become stronger.

With regard to the wake width, Davies found that $\mathrm{L}_{\mathrm{w}} / \mathrm{d}$ increased by about 20 percent for the sychronized D-shaped cylinder vibrating at $\mathrm{a} / \mathrm{d}=0.20$.

By vibrating sma 11 aspect ratio $(L / d=16,20)$ circular cylinders in a uniform flow, Stansby [44] developed empirical formulas to predict the frequency boundaries of the lodk-on region as a function of vibration amplitude in the Reynolds number range $3.6 \times 10^{3}$ to $9.2 \times 10^{3}$. He found that the vortex shedding locked on to submultiples, $\mathrm{f}_{\mathrm{cv}} / \mathrm{f}_{\mathrm{csv}}=2,3$, of the cylinder vibration. At the upper and lower boundaries of locking-on, the regular unforced vortex shedding frequency was intermittent with the locked-on shedding f requency . 
There seems to be no general agreenent as to the effect of synchronized vibrations on the wake width. Sarpkaya [41], in his survey paper, makes the general observation that the overall wake width is practically unaffected in spite of the lateral motion of the body that generates it.

In the synchronization range, there are as yet unexplained discontinuities in the fluctuating forces and pressures, the causes of which are addressed in a recent paper by Zdravkovich [50] - He analyzed flow visualization photographs, which had been previously presented by a variety of authors, seeking to find two different forms of synchronized vortex shedding. If there were two different forms of the synchronized shedding, one in the lower region and another in the upper, then the discontinuities could be explained. Two different forms were found to exist, and he describes them as follows:

(1) In the lower synchronization range, the vortex forms on one side of the cylinder and is shed when the cylinder is close to its maximum amplitude position on the opposite side.

(2) In the upper synchronization range, the vortex again forms on one side of the cylinder. The vortex is shed when the cylinder is near to its maximum amplitude on that same side.

These two forms of shedding were separated by a critical reduced velocity, which corresponded to that value where the discontinuous change of fluctuating and time averaged forces for the forced oscillating cylinder occurred. 
D. Lock-on or Synchronization Phenomena: An overview of studies dealing with changes induced in the near wake of a flexible cylinder or cable by self-excited or forced motion of the body.

Up to now, the background information has essentially dealt with the near wake of stationary or vibrating rigid cylinders in uniform flow. The near wake of a rigid vibrating cylinder in a uniform stream is highly two dimensional because of the increased spanwise correlation and strengthened parallel vortex shedding. The test model used in the present study was a flexible cable. The vortex street wake behind a vibrating flexible cable is now three dimensional because of the spanwise variation in vibration amplitude a/d.

Using hot wire anemometry, Ramberg and Griffin [35, 36, 37] studied the vortex street wake of a forced-vibrating cable in uniform flow for Reynolds numbers between 230 and 1300. The vortex shedding and cable vibration were synchronized throughout all the tests. They determined that the local near wake properties at any spanise location along the vibrating cable behaved similarly to the near wake behind a forced-vibrating cylinder when the frequency, amplitude and Reynolds number were equivalent. Just as Griffin [16] had concluded when he comared the wake flow near self-excited and forced vibrating circular cylinders, they found that the flow in the wake of the forced-vibrating cable could successfully be used to simulate the flow in the wake of a self-excited cable. They examined the local formation length along the span of the vibrating cable, holding the 
vibration frequency constant. The spanwise variation of the formation length was determined by the local amplitude variation. As a/d increased, $L_{f} / d$ decreased, an observation which was consistent with their earlier vibrating cylinder results [16]. An inverse relation was found between the local formation length and vortex strength. This result is consistent with Davies'[10] and Zdrakkovich's [50] observations. This dependence of $L_{f} / d$ on a/d a lso implies that the vorticity generation and lift and drag should depend on the local conditions in the wake. They comared the relative magnitudes of the vortex wake changes behind the cable and rigid cylinder that were induced by synchronization. They noted that the three-dimensional nature of the cable wake had a muting effect (i.e. the magnitude of the changes were smaller). Varying the cable vibration frequency, while holding the shedding frequency (flow velocity) constant, influenced the formation length in the lodk-on region. For vibration frequencies less than the Strouhal frequency, $\left(\mathrm{f}_{\mathrm{cv}} / \mathrm{f}_{\mathrm{cso}}<1\right)$ the formation length is increased, while the formation length decreases when the vibration frequency is greater than the Strouhal frequency.

They defined a wake-capture or frequency-locking Strouhal number $S t_{w}$, which served as a useful means of relating the influence of Reynolds number, vibration amplitude and frequency, on the formation length in the vibrating cylinder or cable near wake

$$
S t_{w}=(1+a / d)\left(f_{c v} / f_{c s o}\right) S t
$$

where

$$
S t=f_{c s o}\left(d / U_{c}\right) .
$$


When $L_{f} / d$ is plotted against $S t_{w}$, the decrease in $L_{f} / d$ with $S t_{w}$ is indicative of the increase in the fluid forces that occur for different conditions of vibration, because a close relation exists between changes in the lift, drag and base pressure on bluff bodies and changes in the formation length caused by vibration.

Ramberg and Griffin [36] then proceeded to examine the vibrating cable wake in order to determi ne the magnitude and spanise extent of correlated vortex shedding along the span of the cable. These observations, as well as measurements of wake velocity profiles, spectra in the flow, and flow visualization studies led them to define three distinct flow regimes along the vibrating cable span in the Reynolds number range 470 to 1300 .

(1) Flow in the immediate vicinity of a cable node closely resembles that past a stationary body. The vortex shedding fluctuations in the near wake occur principally at the Strouhal frequency. Spanwise coherence in the vortex shedding is sma11 or nonexistent. The time-dependent fluid forces resulting from the shedding process near a node are not likely to contribute to the vortex-induced motions of the cable.

(2) The transition region is adjacent to the cable node region and extends to the cable antinode region where the vortex shedding frequency locks-on to the cable vibration. Both the Strouhal and cable vibration frequencies are present in the power spectra of the fluctuating velocity signal from the hot wire anemometer. The spanwise correlation of the shedding increases. An irregular vortex 
pattern, different than the characteristic patterns associated with either the stationary or vibrating locked-on vortex street wakes was observed during flow visualization studies. Only the comonent of the vibration frequency contributes significantly to the motion.

(3) The third region is centered about the cable antinode where the vortex shedding is locked-on to the cable vibration frequency. The spanwise shedding is highly correlated and the power spectra contain a sharp peak at the synchronization, or lock-on, frequency. The degree of correlation was found to be independent of the frequency and amplitude of vibration. The near wake and vortex shedding properties at any point in this region can be represented by those occurring in the wake of a rigid cylinder vibrating at the same Reynolds number, amplitude and frequency. The spanwise extent of this region can be closely approximated by the lock-on regions previously found for rigid cylinders $[25,44]$.

The idea of defining a universal wake Strouhal number for the lodking on of vortex shedding to the vibration of a rigid or flexible cylinder has been explored by Griffin [17]. He presents a model for a universal wake Strouhal number which is valid throughout the entire subcritical Reynolds number range. The model is valid for both forced and vortex-excited oscillations in the lock-on region. The universal Strouhal number can be defined in various ways, depending on what measured wake parameters a re available.

$$
\left.S t *{ }^{*}=\frac{\mathrm{Cs} \mathrm{O}_{\mathrm{L}} \mathrm{w}}{\mathrm{U}_{\mathrm{b}}}=\mathrm{St}\left(\frac{\mathrm{U}}{\mathrm{U}_{\mathrm{b}}}\right)\left(\frac{\mathrm{L}}{\mathrm{d}}\right)=\frac{\mathrm{StL}}{\mathrm{Kd}}=\frac{\mathrm{StL}}{\left(1-\mathrm{C}_{\mathrm{pb}}\right.}\right)^{1 / 2} \mathrm{~d}
$$


where

$$
\begin{aligned}
\mathrm{U}_{\mathrm{b}} & =\text { the velocity just outside the shear layers } \\
\mathrm{K} & =\text { base pressure parameter } \mathrm{K}^{2}=\left(1-\mathrm{C}_{\mathrm{pb}}\right) \\
\mathrm{St} & =\text { usual St rouhal number of the cylinder. }
\end{aligned}
$$

A constant value, $S t^{*}=0.18$, was found for the range of subcritical wake Reynolds numbers, $\operatorname{Re}^{*}=\mathrm{U}_{\mathrm{b}} \mathrm{L}_{\mathrm{w}} / \mathrm{v}$, from 700 to $5.0 \times 10^{4}$. He also noted that the strength of the shed vorticity increased during lockon.

Griffin [19] has published a more recent paper dealing with universal fluid dynamic similarity in the wakes of stationary and vibrating bluff bodies. The wake Strouhal number st ${ }^{*}$ collapses the characteristic wake scales into a curve for wake Reynolds numbers betwe en 100 and $10^{7}$.

\section{E. Stationary and Vibrating Bluff Bodies in Linear Shear Flows.}

Linear shear flows are characterized by the non-dimensional shear parameter $\beta=d / U_{c} d U / d y$. The wake flow behind a stationary bluff body in a linear shear flow is strongly three dimensional. Cross-stream vorticity, the vector of which is normal to the plane of flow, is present in the flow. When these cross-stream vortex lines approach the body, they are turned around the body into the flow direction. The interaction of these rolled-up vortex lines with the Kárman vortex street is believed to be at least partially responsible for the formation of discrete spanwise cells of constant shedding 
frequency in the wake. Maul1 and Young [27] showed that the boundaries between these spanwise cells could be marked by the presence of the rolled-up streamwise vorticity. They placed a sma 11 delta wing, designed to generate a strong longitudinal vortex which would simulate the rolled-up (turned) vortex lines, on each side of a D-shaped cylinder. The spanwise Strouhal number in uniform flow was constant across the cylinder without the delta wings present on the body. When the wings were placed on the cylinder, they caused the spanwise shedding to separate into two cells of different Strouhal numbers, with the boundary between the cells located at the spanwise point where the delta wings produced the longitudinal vortex.

Stansby [44] offers an explanation as to why these discrete cells of constant shedding frequency must exist in a shear flow. The frequency of vortex shedding cannot be constant over the entire bluff body span because that would require a variation in the Strouhal number inversely proportional to the velocity. Vortex shedding must ocar in discrete spanwise cells to preserve the Karman vortex street, the Strouhal number in these cells varying in proportion with the velocity. Mair and Stansby [26] present two ideas as to what may be happening within each of these discrete spanwise cells. They base their ideas on the interaction between the strength of each individual vortex in the Karman vortex street with the cross-stream turned vorticity. In sma 11 cells, the strength of any individual vortex is constant. When larger cells occur, there is an interaction between the strength of the shed vortex and the cross-stream 
vorticity which results in a variation of the strength of the shed vortex along the length of the cell.

The present author puts forth the idea that the lengths of the individual cells in sheared flow are dependent on the formation length value, shear parameter (spanwise velocity gradient), cylinder aspect ratio, and Reynolds number, as well as the vortex strength. End or end plate influence will have a significant effect on the formation region. The author observed this phenomenon during the present set of tests. In the general vicinity of the cable end plates, the formation length increased measurably. It has been noted by Woo et al [48] and Peltzer and Rooney [31] that there were always stable cells in sheared flow adjacent to the end plates of high aspect ratio cylinders, even though the central span exhibited a slight tendency or no tendency towards forming a cellular structure.

Returning to Figure 1 and considering the data of Woo et a1 and Peltzer only, one observes that as the Reynolds number increases from $10^{3}$ to $10^{4}$, the formation length decreases, and as the Reynolds number increases further, the formation length remains constant at a minimum value. When all the experimental studies [31, 32, 47] dealing with the vortex wake behind high aspect ratio cylinders $(\mathrm{L} / \mathrm{d}>27)$ in a shear flow are analyzed, they reveal an interesting correlation between the length of the formation region and the existence of stable cells. The results of Woo et al show that some type of cellular vortex structure existed in the wake of their cylinders up to Reynolds numbers around $10^{4}$. Peltzer and Rooney's 
results show that no measurable spanwise cellular pattern existed from Reynolds numbers of $2 \times 10^{4}$ to $1.1 \times 10^{5}$. It seems that there is a minimum formation length, below which the cell boundaries fluctuate wildly or no cells exist at al1. As the formation length decreases, the length and stability of the cells decreases. To strengthen this observation, the author observed that when the centerline Reynolds number was increased from $3.0 \times 10^{3}$ to $9.0 \times 10^{3}$, (first phase cable studies in sheared flow) the cells at $9 \times 10^{3}$ were measurably smaller than those at $3.0 \times 10^{3}$. The shear parameter $B=0.0053$ was held constant and only the Reynolds number was varied.

With regard to sma 11 aspect ratio $(\mathrm{L} / \mathrm{d}<20)$ effects, the tendency for discrete stable cells to form is much greater on a small aspect ratio cylinder than it is for a high aspect ratio cylinder under the same flow conditions. Both Maull and Young [27] and Mair and Stansby [26] have exami ned the effect of a sheared flow on the spanwise Strouhal number distribution of small aspect ratio bluff bodies $(\mathrm{L} / \mathrm{d}<20)$. Both found that a number of coherent cells were present along the span of their models for Reynolds numbers less than $2 \times 10^{4}$. Mair and Stansby determined an optima 1 end plate design to stabilize the vortex shedding cells in the wake of their low aspect ratio models. They noted that without this design, the results showed a continuous change in peak frequency which was attributed to unstable cell boundaries (i.e. short $L_{f}$ ). The increased formation length due to the presence of the end plates was the stabilizing factor, such that coherent stable cells were allowed to form. 
For flows with a large value of the shear parameter $\beta$, the tendency towards forming a stable spanwise cellular structure in the wake is much greater than that for a weakly sheared flow. Both Woo et al and Peltzer [32] have noted this. The higher shear level flows contain a larger amount of streamise vorticity which helps to establish and stabilize cells and cell boundaries. Both authors have shown that the length of the discrete cells in a sheared flow varies in inverse proportion to the shear parameter $\beta$ Peltzer has shown that the change in Strouhal number between consecutive cells varies in proportion to the shear parameter $\beta$.

The work by Stansby [44] represents the only experimental study dealing with the wake properties behind a vibrating ciralar cylinder in a sheared flow. The vortex shedding frequency locked-on to the cylinder vibration frequency, as we 11 as to submitiples of it, over a portion of the cylinder span. The rest of the cells that were unforced (not locked-on) became very stable in frequency (i.e. the spanwise shedding structure became very well defined). He showed that the uniform flow results were similar to the shear flow results and that they could be applied to the lock-on problem in shear flows.

Woo et al studied the vortex wakes of oscillating cables in a linear shear flow. They discuss the mechanism of complete locking-on based on the concept of the modulation of the vortex shedding process due to the forced oscillation of the cable. They found that the body oscillations induced vortices which were in perfect synchronization with the body motion, and that the strength of these vortices was 
amplified. The unforced cells adjacent to the lock-on region were stabilized. The frequencies of the shed vortices in those regions adjacent to the lock-on region were forced away from those values corresponding to the stationary cable shedding frequencies (in sheared flow). Finally, based on their experimental results, they established a procedure to estimate the spanwise extent over which the vortex shedding locks on to the oscillations of a cable in sheared flow.

Two excellent review articles dealing with flow around stationary and vibrating bluff bodies have recently been published. The research topics discussed in Sarpkaya's [41] review paper on vortex-induced oscillations include vortex shedding from a stationary bluff body; consequences of the synchronization phenomenon; wake oscillator models; added mass, daming, and dynamic response measurements; flow field models and the discrete vortex method; mechanics of synchronization; and in-line oscillations. Griffin et a1 [20] present an overview of the state of knowledge of strumming oscillations and apply these findings to the development of design methods for cable systems likely to undergo these oscillations. The report is limited in scope to the problems caused by vortex shedding from bluff flexible structures and cables in steady currents, and the resulting vortex-excited oscillations. 


\section{F. Vortex Shedding From a Sphere}

To the best knowledge of the author, no experimental studies or theoretical analyses have been attempted that consider the wake interactions behind a stationary or vibrating cable in sheared flow with bluff body shapes attached. In the present study, spherical bluff body shapes were positioned at various equidistant spanwise locations on the cable, and the near wake properties of the spherecable bluff body combinations we re studied.

It is appropriate to discuss the vortex wake structure of a sphere at this time. In the Reynolds number range $10^{3}$ to $2.0 \times 10^{5}$, the drag coefficient is nearly constant at a value $C_{D}=0.45$. Using this value, and Figure 7, pp. 3-6 of Hoerner [24], the value of the Strouhal number is a constant, $S t_{S}=0.37$. Pao and Kao [29] towed a sphere through a weakly stratified fluid at constant velocity and measured the Strouhal number in the Reynolds number range $4.3 \times 10^{3}$ to $1.74 \times 10^{4}$. They present a tabular summary of experimental values of the Strouhal number of a sphere measured by different investiga tors.

$\begin{array}{ccccc}\text { Re } & \text { Pao \& Kao [29] } & \text { Achenbach [1] } & \text { Cometta } & \text { Winny } \\ 4300 & 0.14 & 0.125 *(6000) & 0.19 & 0.71 \\ 7800 & 0.18 & 0.14 & 0.19 & 0.55 \\ 17400 & 0.22 & 0.17 & 0.19 & 0.20 *(13000)\end{array}$

The starred values indicate that the Reynolds number corresponding to the measured Strouhal number was that shown in parentheses. 
In the present set of tests, one of the goals was to determine the Strouhal number of the spherical bluff body shapes. The cable extended through the central one-third diameter of the sphere during these measurements and it was expected that this arrangement would change the expected physical properties and near wake conditions normally associated with a sphere in uniform flow. It was also assumed that the vortex shedding from the sphere would lodk-on to the forced vibration of the sphere in a manner similar to that exhibited during cylinder lock-on.

Vortex shedding in the fully turbulent near wake of a sphere is three dimensiona 1. Achenbach [1] found that the vortex separation point rotated around the sphere for Reynolds numbers above $6.0 \times 10^{3}$; however he could not describe or model the vortex wake structure. Pao and Kao suggested that the separation point movement implied that the vortex wake configuration would be a double helical vortex loop. At the initiation of the vortex shedding process, the double helix must form a closed-end loop in the fluid. The vorticity vector is continuous along the loop, such that the vorticity vectors in the two branches at any cross section perpendicular to the wake axis are opposite in sense. Thomson's circulation theorem, which states that the net flux of vorticity across planes perpendicular to the wake axis must be zero, is satisfied. The position of the rolling up of the vortex sheet is 0.5 diameters behind the sphere at $\operatorname{Re}=3.0 \times 10^{3}$, and Achenbach assumes that the position of this rolling up occurs near the surface of the sphere at $\operatorname{Re}=6.0 \times 10^{3}$. 
G. Procedure Used to Design a Workable Experimental Test Setup.

Before considering any type of experimental analyses, a workable experimental design that would satisfy all the limiting constraints imposed by the wind tunnel specifications, cable properties, and available experimental apparatus had to be formulated. A basic relationship that would relate the natural frequencies and mode shapes to the length and tension of the cable and the flow speed was needed. Treating the cable as a continuous system and applying Newton's second law for the forces in the vertical direction on a small spanwise element dy, one obtains the vertical equation of motion for the cable. (This type of analysis can be found in many classical vibration textbooks: one example is Elements of Vibration Analysis by Leonard Meirovitch, McGraw-Hi11 Inc., 1975, pp. 193195). The end conditions are fixed-fixed, no bending stiffness terms are included (ideal cable), and the oscillations are assumed to be sma11. Solving the eigenvalue problem associated with the cable system having fixed-fixed end conditions, the appropriate relationship between the natural frequencies of the idealized cable system and the cable length and tension, and wind tunnel velocity has been found. The natural frequencies of the system $\omega_{n}$ are:

$$
\omega_{\mathrm{n}}=\frac{\mathrm{n} \pi}{\mathrm{L}}\left(\frac{\mathrm{T}}{\rho}\right)^{1 / 2} \mathrm{n}=1,2,3, \ldots, \infty
$$


In this study the first and second modes we re employed, $(n=1,2)$ so the natural frequencies were:

$$
w_{1}=\frac{\pi}{L}\left(\frac{T}{\rho}\right)^{1 / 2}, \quad w_{2}=\frac{2 \pi}{L}\left(\frac{T}{\rho}\right)^{1 / 2} .
$$

$T$ is the cable tension $\left(1 b_{f}\right)$ and $\rho$ is the mass density of the cable $\left(1 b_{f} s e c^{2} / \mathrm{ft}^{4}\right)$. The specific gravity (SG) and diameter of the cable were provided in the specifications. The mass density then becomes:

$$
\rho=S G(1.94) \frac{\pi d^{2}}{4} \text { slug } \mathrm{ft}^{2}
$$

The natural frequencies $w_{1}$, $w_{2}$ are related to the cable vortex shedding frequency $\mathrm{f}_{\mathrm{cso}}$ as follows:

$$
\omega=2 \pi \mathrm{f}_{\operatorname{cso}}{ }^{\circ}
$$

The vortex shedding frequency is related to the Strouhal number (which was assumed constant in the Reynolds number range covered by these tests) by:

$$
\mathrm{f}_{\mathrm{cso}}=\frac{\mathrm{stU_{c }}}{\mathrm{d}}
$$

Substituting all these values into the equation for the natural frequencies of the system, one obtains the desired relationship:

$$
\frac{\mathrm{T}}{\left(\mathrm{LU}_{\mathrm{c}}\right)^{2}}=\mathrm{SG} \pi 1.94\left(\frac{\mathrm{St}}{\mathrm{n}}\right)^{2}
$$


The flow velocity, $U_{c}$, is fixed between 8 and 80 fps because Reynolds numbers of order $10^{3}$ are desired. The specific gravity of the cable is 1.2 , and the Strouhal number is 0.19 . The tension was limited to values between 100 and $10001 b_{f}$ by the load ce11's measuring capacity. The working length of the cable could be varied between three feet and five feet while still retaining complete traverse coverage and a high aspect ratio. The inability to reproduce the proper reduced cable damping in air necessitated the artificial (motor-driven) oscillation of the cable. The maximum motor speed was around $100 \mathrm{~Hz}$ when the eccentric mass was attached to the shaft. After the various combinations of cable length and tension, and vibration frequency (vortex shedding frequency or flow velocity) were analyzed, it was decided to design the experinent using the following values for the various parameters

Cable Length: $\mathrm{L}=4 \mathrm{ft}$.

Tension: First mode $\mathrm{T}=5001 \mathrm{~b}_{\mathrm{f}}$, Second Mode $\mathrm{T}=2501 \mathrm{~b}_{\mathrm{f}}$

Flow Velocity: Could be varied from 8 to 80 fps

Motor Speed: First mode $55 \mathrm{~Hz}$, Second Mode $78 \mathrm{~Hz}$.

\section{H. A Brief Discussion of the Fast Fourier Transform} and Related Topics (Power Spectra)

The Fourier Transform serves as a bridge between the time domain and frequency domain. When the fluctuating sinusoidal or random time based signal is to be analyzed on a digital compter, the Discrete Fourier Transform (DFT) is used. The Fast Fourier Transform (FFT) is 
an efficient, high speed method used to compute the DFT. The frequency spectrum is calculated from the sampled time function, rather than measured using a single fixed-band-pass filter. The first major step involved with using the FFT procedure is digitization of the recorded analog signal $x(t)$. There are two processes associated with the digitization of an analog signal $x(t)$ (1) sampling defines the instantaneous points at which the data are to be observed; (2) quantization converts the data values at the sampling points into nume rical form.

Sampling of an essentially stationary continuous $x(t)$ vs. $t$ record for digital analysis is performed at equally spaced intervals, $\Delta$. The appropriate sampling interval $\Delta t$ must be chosen such that no allasing, confusion between low and high frequency (multiples) components in the original data, is allowed to occur. The sampling rate is then $1 / \Delta t$ samples/sec. The highest frequency that can be defined is $1 / 2 \Delta t$, because at least two samples/cycle are required to define a frequency component in the original data. This frequency, $1 / 2 \Delta t$, is called the Nyquist or folding frequency $f_{c}$. To help avoid aliasing, $f_{c}$ should be chosen to be one and one half to two times greater than the maximum anticipated frequency. Low pass (antialiasing) filtering must be used to elimi nate aliasing.

Only a finite number of samples of $x(t)$ vs. $t$ at the intervals $\Delta t$ can be taken and stored. This finite number, $N=1024$ consecutive nonzero samples, defines the time length of the required data record, $\mathrm{T}_{r}=\mathrm{N} \Delta \mathrm{t}$. The values of $\Delta t$ most frequently chosen in 
the present study were $\Delta t=2$ and $5 \mathrm{mi} 11$ iseconds, such that the data record time lengths were 2.05 and 5.12 seconds, respectively. The spectral resolutions (resolution bandwidths) $\Delta \mathrm{f}=1 / \mathrm{T}_{\mathrm{r}}$ defined by these two $\Delta t$ interval values a

In the present study, the Zonic FFT with a DMS 5003 computer was used to calculate the power spectral density function, which decribes the general frequency composition of the random stationary data. The power spectrum is computed by taking the sum of the squares of the real and imaginary components of the Fourier coefficients of the DFT. For random stationary data, a true spectrum exists and can be obtained by averaging many individual spectra. The Zonic FFT possesses the capability to average individual spectra. It is noted in the literature, that reasonable statistical quality requires averaging of 64 to 100 individual spectra. This would require that 131 to $205 \mathrm{sec}$, or 328 to $512 \mathrm{sec}$ of data be taken when $\Delta t=2$ or 5 millisec. respectively. A very large number of these spectra (approximately 700) were taken during the two major weeks of wind tunnel tests. In order for each of the spectra to be statistically valid, 60 to 82 hours of running tine would have been required. The 1imitations on available wind tunnel time, funding for the research, and human endurance, required that the number of averages be reduced to $30(\Delta t=2$ millisec.) or $20(\Delta t=5 \mathrm{millisec})$. Power spectra, using 30 and 100 or 20 and 100 averages, were then comared to observe whether any significant differences existed between them. It was found that the fairly narrow-band, high energy peaks defining the 
vortex shedding and vibration frequencies were identical on both spectra. The only significant difference between the two spectra, was that the small fluctuations in the spectral plots in the areas away from the peaks were slightly less when 100 averages were used. Twenty and thirty averages were then sufficient to give an accurate statistical measure of the shedding and vibration frequencies. 
III. Experimental Ap paratus and Instrumentation

\section{A. Wind Tunnel Test Facility}

Al1 experiments were conducted in the $28 \mathrm{ft} .(8.5 \mathrm{~m})$ 1ong, $6 \mathrm{ft}$. $(1.8 \mathrm{~m})$ square, test section (Figure 3 and 4 ) which is part of the VPI\&SU continuous, closed jet, single return, subsonic stability wind tunne1. The test section was designed such that any combination of six different wire screens can be positioned in its upstream portion. These various combinations of screens generate numerically unique linear velocity shears ( $d U / d y$ ) across the span of the test section. The numerical values of these velocity shears were characterized by the nondimensional shear parameter, $\beta=d / U_{c} d U / d y$. Spanwise velocity and turbulence profiles, corresponding to the two shear levels $(\beta=0.0$ and 0.0053$)$ used in the present study are shown in Figures 5 and 6 . The turbulence intensity (Tu\%) is defined as the ratio between the measured rms value of the fluctuating velocity signal from the hot wire anemometer and the steady dc value of the velocity. The velocity profiles are plotted as a dimensionless ratio $U / U_{c}$, where $U$ is the local velocity and $U_{c}$ is the centerline velocity, vs. y/d. Both the velocity and turbulence profiles are uniform in unsheared flow. The flow of $0.25 \%$ turbulence was generated by placing a wire screen of uniform crosstunne1 distribution at the upstream end of the test section. The velocity decreased in a very linear fashion across the span when the shear leve 1 was increased to $\beta=0.0053$ by placing five screens of nonuniform cross-tunnel wire distribution at the upstream end of the 
test section. The turbulence increased near the low velocity end because the density of wire screens increased in order to generate the low velocity.

To monitor the tunnel velocity, a pitot-static tube, connected to both the VPI\&SU Digital Dynamic-Pressure Indicator and Barocel Electronic Manometer, was used. The pitot-static tube was mounted to the test section floor at the spanwise center of the tunnel, extending vertically into the freestream. The static freestream pressure was measured by a Validyne Digital Barometer Model DB99. The freestream temperature was monitored by a temperature probe which was mounted to the test section wa11. The temperature value was continuously displayed by a VPI\&SU Digital Thermometer.

An HP 3052A Data Acquisition System was located in the control room, adjacent to the test section. The five major comonents of the system included an HP 9835B Desktop Computer, an HP 3456A Digital Voltmeter, an HP 3437A System Voltmeter, an HP 3497A Data Acquisition/Control Unit, and a 30 channel system scanner. The pitot static tube values, the freestream temperature values, and the static freestream pressure values were all hard wired to the system scanner. Programs to constantly monitor the tunnel velocity and Reynolds number were avallable through the computer.

A three-dimensional traverse (see Figures 3 and 4) was available to transport the measuring instruments (Physitech Optical Head and hot wire probe) in the vertical ( $x / d)$, spanwise (y/d) or streamise (z/d) directions. The center of this axis system is located 
vertically at the cable center axis, positive $x / d$ up; spanwise at the wind tunnel and cable centerline, positive $y / d$ to the right (looking upstream); and streamwise at the center axis of the cable, positive $z / d$ downstream. The spanwise ( $y / d$ ) motion was controlled by a 15 volt to 30 volt dc gearhead motor, which was powered by an HP 6255A 0-40 V Dual DC Power Supply. The spanwise motion was displayed by a digital counter, accurate to $\pm 1 / 100$ inch $( \pm 1 / 40 \mathrm{~cm})$. Two precision step motors were used to drive the vertical ( $x / d)$ traverse. The control box was specially designed for that purpose at VPI\&SU. The vertical position was also displayed digitally and was accurate to $\pm 1 \mathrm{~mm}$. The streamise $(z / \mathrm{d})$ traverse was driven by a $12 \mathrm{~V}$ to $24 \mathrm{~V}$ DC gearhead motor, powered by the other side of the HP 6255A Dual DC Power Supply. The spanise position was monitored by a Clarostat Model 73JA ten turn linear potentiometer, powered by a Lambda Mode1 LM2035 V DC Power Supply. The digital output from the potentiometer, which corresponded to the spanwise position, was displayed on an HP 3476B Multimeter. The spanwise positions were accurate to $\pm 1 / 80$ inch $( \pm 0.32 \mathrm{~mm})$.

\section{B. Test Mode1}

The cable that was used in the tests was manuactured by Philadelphia Resin Corporation, and was labeled "Phillystran" ps $29-C-39 J . \quad$ It was constructed of seven strands of "Kevlar" ps 29-B105 rope, wound around each other helically, and wrapped with a polyurethane jacket. The cross section was not circular. The 
cable had a $3 / 8$ inch $(9.53 \mathrm{~mm})$ nominal diameter, and a 0.45 inch $(11.43 \mathrm{~mm})$ measured diameter over the jacket. The specific gravity of the cable was approximately 1.2, and the breaking strength was $17,0001 b_{f}$

\section{Experimental Test Setup}

Figures 3 and 4 show a top and upstream view of the testing equipment arrangement. The cable was connected to the turnbuckles, which were attached to the I-beam support frame, and stretched across the span of the tunne1. The center axis of the cable was at a vertical height, $x / d=0$, midway between the test section floor and ceiling. It was decided that a working distance of $\mathrm{L}=4$ feet $(1.22 \mathrm{~m})$ between the end plates would be optima 1. The resulting aspect ratio was $\mathrm{L} / \mathrm{d}=107$. The cable was securely fastened (pinned) at the end plates which were supported by braces extending between the test section ceiling and floor. This arrangement ensured that no vibration would be transmitted beyond the end plates. The cable tension was adjusted using two $3 / 8$ inch $(9.53 \mathrm{~mm})$ turnbuckles which were located outside each of the test section walls. The cable ends were secured to the turnbuckles by four $3 / 8$ inch $(9.53 \mathrm{~mm})$ cable clamps. The turnbuckle at the left wa 11 was attached directly to the welded I-beam support structure. The 6 inch I-beam support structure was laid across the top of the test section. The right turnbuckle was attached to a Strainsert, Model FLlU, 1000 1b, Single Bridge, Universal Flat Load Ce11, which was bolted onto the I-beam support. 
The load cell was used to constantly monitor the cable tension (Figure 7). The calibration factor was $3 \mathrm{mv} /(\mathrm{V}$ excitation) output. The 15 V DC excitation voltage was supplied to the load cell by a Weston Model 301 0-15 V DC Power Supply. The output (now calibrated such that $45 \mathrm{mv}$ was equal to $10001 \mathrm{~b}_{\mathrm{f}}$ voltage was digitally displayed on an HP $3476 \mathrm{~B}$ Multimeter. The value of the cable tension could be measured accurately to within \pm 1 percent.

It was initially determined that the cable was too highly damed to be naturally excited into the lowest vibrational modes by adjusting the wind speed. A small Radio Shack 0-6 V DC Mabuchi Motor, with an eccentrically located mass was attached to the cable at $y / d=43.1$, near the right end plate. Both the first and second modes of vibration could be excited by varying the motor speed. Figure 8 shows the forced cable vibration setup. An HP 6255A Dual DC $0-5 \quad V$ Power Supply provided the variable motor excitation voltage. The DC excitation voltage was constantly monitored on the HP $3476 \mathrm{~B}$ Multimeter

D. Cable Mode Shape Vibration Amplitude and Frequency Measurement

A Physitech Model OH39B Remote Optical Head was mounted to the traverse (downstream of the cable) and focused on the top edge of the vibrating cable. An incandescent light source was placed in front of the cable (upstream). The optical head was connected to a Physitech Mbdel 440 Electro Qptical Auto Collimator Control Unit (Figure 9). A general non-technical description of the measuring system principle 
associated with the optical head, auto collimator, incandescent light source system follows [33]. The optical head with a suitable lens system focused an optical discontinuity (cable-background light interface) on the light sensitive area of the photomultiplier tube within the optical head. The focused image (cable) represented a target whose movement was to be measured. The optical image was converted to an identical electron image by the photomultiplier tube. The control unit sent a repetitive electrical signal to the deflection yoke in the image analyzer. This signal caused the photomultiplier tube to scan the electron image in a predictable manner, moving across the interface in a perpendicular axis. As the scan moved across the interface, the output of the photomultiplier tube sharply changed. The deflection signal current was proportional to the position of the scan at any given time. The change in the photomuliplier tube output instantly caused the control unit to sample this current level, and thereby determined the position of the interface with respect to the deflection current. Since the movement of the interface was equivalent to the physical movement to be measured, the sampled level which occurred when the interface was "crossed" was the position of the target being measured.

To measure the amplitude of vibration, the output voltage from the auto collimator unit (calibrated such that $1 \mathrm{~mm}=2 \mathrm{~V} \pm 0.1 \mathrm{~V} \mathrm{DC}$ using the gain setting) was sent to a Disa Type 55D35 True RMS Voltmeter. The rms reading was then a direct measure of the amplitude. In order to determine the vibration frequency, the output 
signal from the auto collimator unit was band-pass filtered in a Krohn Hite Model 335R Variable Band-pass Filter and sent to a ZTL Inc. Multichannel FFT Processor with DMS 5003 Computer. The Krohn-Hite was used as an antialiasing filter. The FFT was used to calculate a power spectrum of the fluctuating signal. The vibration frequency and power spectrum were then displayed on the Tektronix 4010 Graphics Display Terminal. The time based signal was continuously displayed on a Tektronix 5103N oscilloscope, thereby providing a visual monitoring of the cable vibration characteristics.

\section{E. Vortex Shedding Frequency Measurement}

A TSI Model 1210 straight hot wire probe was attached to the traverse and positioned in the cable wake at the point where the strongest vortex shedding occurred. The 0.0015 inch (3.8 $\mu \mathrm{m})$ platinum plated tungsten wire sensor was operated at an overheat ratio of 1.8 using a TSI Model 1050 Constant Temperature Anemometer module with a TSI model 1051-6 Power Supply and Monitor (Figure 10). The output signal from the a nemometer was band-pass filtered in a Krohn-Hite Model 335R Filter, and sent to the ZTL Inc. Multichannel FFT Processor with DMS 5003 Computer. The FFT was used to calculate a power spectrum of the hot wire signal. The power spectrum was then displayed on the Tektronix 4010 Graphics Display Terminal, as we 11 as the numerical value of the vortex shedding frequency which was obtained using the peak search command within the FFT. The display terminal screen was photographed by a Graflex camera loaded with 
Polaroid Type 57 High Speed Film. The hot wire signal was displayed at a11 times on the Tektronix 5103N Oscilloscope.

\section{F. Cable Wake Width and Vortex Formation Length Instrumentation}

The TSI Model 1210 hot wire probe was mounted to the traverse. It was then positioned either directly behind the base of the cable for formation length $\left(L_{f}\right)$ measurements, or one diameter above the top of the cable for wake width $\left(L_{W}\right)$ measurements. Using this arrangement it was then possible to traverse downstream in the streamwise direction to measure $\mathrm{L}_{\mathrm{f}}$, or across the cable wake to measure $\mathrm{L}_{\mathrm{w}}$. The probe was operated at an overheat ratio of 1.8 using the TSI Model 1050 constant temperature anemometer module with a TSI Mode1 1051-6 Power Supply and Monitor (Figure 11). The output signal from the anemometer was band-pass filtered in a Multimetrics Inc. Mode1 AF-420L Active Filter. For formation length traces, the low and $\mathrm{high}$ pass frequencies were $2 \mathrm{f}_{\mathrm{csv}} \pm \mathrm{f}_{\mathrm{csv}} / 10$. The high and low pass frequencies for wake width measurements were $10 \mathrm{~Hz}$ and $\left(2 \mathrm{f}_{\mathrm{csv}^{-}}\right.$ $\left.f_{c s v} / 2\right)$. The rms value of the filtered hot wire signal was displayed on the Disa Type 55D35 True RMS Voltmeter. Graphical displays of both the rms $\mathrm{L}_{\mathrm{f}}$ and $\mathrm{L}_{\mathrm{W}}$ traces were provided by an HP Model $7100 \mathrm{~B}$ Strip Chart Recorder. Three second averaging was used to smooth out the fluctuations in the rms curves. 
IV. EXPER IMENTAL METHODS

\section{A. Cable Mode Shape and Vibration Frequency Measurement}

It was decided that a cable tension of $5001 b_{f}(0.0225 \mathrm{~V}$ output from the load ce11) would be optimal to use for all first mode forced vibration tests. The natural frequency of vibration was estimated to be around $60 \mathrm{~Hz}$. The cable tension was set to a value slightly higher than $5001 b_{f}$ and as the cable stretched the tension slowly decreased, finally settling in at a value close to $5001 \mathrm{~b}_{\mathrm{f}}$. The Physitech Remote Optical Head was mounted to the spanise traverse at a downstream distance, $z / d_{c}=44.5$. The lens system was then focused on the interface between the upper edge of the cable and the incandescent light source which was placed upstream of the cable. This location was established as the zero deflection position by adjusting the zero set on the auto collimator control unit. The control unit was calibrated such that $1 \mathrm{~mm}$ of static deflection was equal to $2 \mathrm{~V}$ de output. This calibration was accomplished by deflecting the vertical traverse $1 \mathrm{~mm}$ and increasing the gain setting on the control unit until the voltage reading on the multimeter was equal to $2 \mathrm{~V}$ dc.

The signal output from the control unit was simultaneously sent to a Disa rms meter, oscilloscope and Zonic FFT. The voltage supplied to the sma 11 motor with the eccentric mass was adjusted such that the cable was forced to vibrate at its maximum amplitude in the first mode. The Physitech output signal was band-pass filtered in the Krohn-Hite filter to prevent any signal aliasing. The FFT was 
used to calculate a power spectrum of the filtered signal. The peak search command was then used to obtain the numerical value of the cable vibration f requency .

After the natural frequency of vibration of the cable was determined, the optical head was moved to the spanwise location, $y / d=-52.0$. The optical head was traversed across the span of the cable, from $y / d=-52.0$ to $y / d=40.0$, in increments of $y / d=4.0$. At each increment, the rms value of the vibration amplitude was measured. When each of these rms values is nondimensionalized by the maximum rms value at $\mathrm{y} / \mathrm{d}=0$, an accurate representation of the mode shape can be plotted.

The same type of procedure was used to obtain the mode shape and natural vibration frequency associated with the cable when it was forced to vibrate in the second mode. The cable tension was decreased to $2251 b_{f}(0.0101 \mathrm{Vdc}$ output from the load cell) and the second mode natural frequency was estimated to be around $67 \mathrm{~Hz}$. The span of the cable was again traversed in increments of $y / d=4.0$, and the rms value of the signal at each location was obtained. The FFT was used to calculate the second mode natural vibration frequency.

\section{B. Cable Lock-on Region Tests}

Prelimi nary observations of power spectra, taken of the hot wire signal at various combinations of vertical and streamwise positions above and behind the base of the cable, indicated that the position of the strongest vortex shedding was at a height, $x / d=0.92$ and 
downstream location, $z / d=3.5$. Before any lock-on tests were performed, the Strouhal number of the non-vibrating cable as a function of increasing Reynolds number was determined. The hot wire probe tip was placed at the optimal position $(\mathrm{x} / \mathrm{d}=0.92, \mathrm{y} / \mathrm{d}=0$, $z / d=3.5)$ and the filtered signal from the anemometer was input into the FFT. A power spectrum of the signal was calculated and the vortex shedding frequency was found using the peak search command, and displayed on the terminal screen. The corresponding Reynolds number was calculated by the data acquisition system program and printed out along with the centerline velocity. The Strouhal number was then calculated using the relationship, $S t=f_{c s} d / U_{c}$. The Reynolds number range covered by these tests was $1.8 \times 10^{3} \leq \operatorname{Re}_{c} \leq 41.0 \times 10^{3}$.

Again, preliminary observations of power spectra of the hot wire signal were used to help establish the criteria that would determine when the vortex shedding frequency from the cable ( $f_{c s v}$ ) locked-on to the forced vibration frequency of the cable $\left(f_{c v}\right)$. When the amplitude ratio of the vibration and vortex shedding peaks was equal to $20\left(a_{\mathrm{cv}} / a_{\mathrm{csv}}=20\right)$, the vortex shedding was considered locked on to the forced cable vibration. The frequency range over which the cable vortex shedding locked on to the cable vibration, as a function of vibration amplitude $(a / d)$, was determined using the following procedure. The cable was forced to vibrate in the first mode and the Physitech optical head was used to measure the vibration amplitude $(\mathrm{a} / \mathrm{d})$ at $\mathrm{y} / \mathrm{d}=0$. The hot wire was placed at the optimum position 
$(\mathrm{x} / \mathrm{d}=0.92, \mathrm{y} / \mathrm{d}=0, \mathrm{z} / \mathrm{d}=3.5)$. The Reynolds number was gradually increased until the lower lock-on frequency was determined (vortex shedding locked on to the cable vibration), and increased until the upper lock-on frequency was established (vortex shedding broke away from the cable vibration). This procedure was repeated at spanise intervals of $y / d=4.0$, starting at the center of the cable, $y / d=0$, and ending at the left end of the cable, $y / d=-48.0$. The same procedure was followed using the second mode shape. In this manner, the variation of the lock-on region as a function of increasing amplitude of vibration (a/d) was studied.

When the lock-on range vs, a/d tests were comleted, the probe was positioned at $\mathrm{y} / \mathrm{d}=0$. With the cable still vibrating in the first mode, the variation of the Strouhal number (cable vortex shedding frequency) as a function of increasing Reynolds number was determined, so that these values could be compared to the stationary cable St vs. Re data. Another basic test was also conducted. The spanwise variation of the vortex shedding frequency from a vibrating cable, at Reynolds numbers preceding and following the locked-on region was examined. It was desired to determine if the cable mode shape, (i.e., spanwise variation in a/d due to the vibration) had any effect on the spanwise shedding frequencies. To accomlish this, the cable was vibrated in the first mode and the entire span of the cable, $-48.0 \leq y / d \leq 40.0$, was $t$ raversed in increments of $\mathrm{y} / \mathrm{d}=4.0$. At each spanwise location, a power spectrum of the filtered hot wire signal was calculated, and the nume rical value of 
the cable vortex shedding frequency was obtained using the FFT's peak search command.

\section{Vortex Formation Length $\left(L_{f}\right)$ and Wake Width $\left(L_{W}\right)$}

The criterion used to determine the location of the vortex formation length $\left(L_{f}\right)$ was that outlined by Bloor and Gerrard [8] and Ramberg [34], i.e., the maximum of the second harmonic of the fluctuating velocity on the wake axis. To measure $L_{f}$, the probe tip (mounted to the 3-D traverse) was positioned parallel to the center axis of the cable $(x / d=0)$ at a downstream distance, $z / d=0.92$. The probe was traversed in the streamwise (downstream) direction. A graphical display of the filtered rms formation length trace was provided by the strip chart recorder. The variation of the formation length with increasing Reynolds number behind a non-vibrating cable was investigated first. The cable was vibrated in the first mode and the amplitude at $\mathrm{y} / \mathrm{d}=0$ was measured using the Physitech optical head and rms meter. The $\mathrm{L}_{\mathrm{f}}$ trace was obtained at the lowest Reynolds number, $R e=2.0 \times 10^{3}$. The Reynolds number was gradually increased in sma 11 increments until Re $=42.0 \times 10^{3}$. At each of these Reynolds number increments, the $\mathrm{L}_{\mathrm{f}}$ trace was recorded. The amplitude at $\mathrm{y} / \mathrm{d}=$ 0 was then changed and the same procedure repeated. Four different a/d values were tested.

Once the formation lengths behind a non-vibrating and vibrating cable were obtained, the probe position was changed in order to measure the wake width. The variation of $L_{w}$ with increasing Reynolds 
number behind a stationary cable was investigated as follows. The probe was positioned at the correct value of $L_{f}$ corresponding to the Reynolds number of the test being run. The probe was raised to a position, $x / d=1.0$, and then traversed through the wake of the cable to the vertical position, $x / d=-1.0$. The strip chart provided a graphical display of the filtered rms velocity trace ( $L_{w}$ trace) across the wake. The distance between the two maxima in the profile defined the wake width, $\mathrm{L}_{\mathrm{W}}$. The Reynolds number range for all the wake width tests was $2.0 \times 10^{3} \leq \operatorname{Re} \leq 42.0 \times 10^{3}$.

Once the wake widths were determined as a function of increasing Reynolds number for the non-vibrating cable, the cable was vibrated in the first mode at each of those $\mathrm{a} / \mathrm{d}$ values corresponding to the formation length traces. Following the same procedure used to determine the variation of $\mathrm{L}_{\mathrm{W}}$ with increasing Reynolds number for the non-vibrating cable, the variation of $L_{w}$ with increasing Reynolds number at each individual value of a/d for the vibrating cable was investigated. The wake width traces were displayed on the strip chart recorder.

\section{Sphere Lock-on Regions}

One of the goals of this study was to analyze the effect that placing various bluff body shapes on the cable would have on the spanwise variation of vortex shedding from both a stationary ( $\mathrm{fsso}_{\text {s }}$ ) and vibrating $\left(f_{s s v}\right)$ cable in sheared and unsheared flow. The shape chosen was a 1.5 inch $(3.81 \mathrm{~cm})$ diameter spherical ping pong ball. 
The balls had 0.45 inch $(1.14 \mathrm{~cm})$ diameter holes drilled through them so they could be mounted on the cable at various $y / d$ locations. Ping pong balls were chosen because their mass insignificantly affected the cable mode shape characteristics, i.e., amplitude and vibration f requency •

Before investigating the lodk-on regions for the sphere, the behavior of the vortex shedding (St) from the sphere as a function of increasing Reynolds number was investigated in the range

$6.57 \times 10^{3} \leq \operatorname{Re} \leq 24.17 \times 10^{3}$. The hot wire probe was positioned at $x / d=0.92$ and $z / d=3.5$, directly behind the base of the sphere. The Reynolds number was increased in small increments, and at each increment a power spectrum of the filtered hot wire signal was calculated. The shedding frequency $\left(\mathrm{f}_{\text {sso }}\right)$ of the sphere was found using the FFT's peak search command and displayed on the terminal screen.

The criterion that was used to determine when the vortex shedding from the sphere ( $\left.f_{s s v}\right)$ locked-on to the vibration frequency of the sphere $\left(f_{S v}\right)$ was slightly different than that used to determine the cable lock-on frequencies. When the amplitude ratio of the vibration and vortex shedding peaks was equal to $10\left(a_{s v} / a_{s s v}=10\right)$, the shedding frequency of the sphere was considered to be locked on to the vibration frequency of the sphere. The two criteria were different because the amplitude of the cable vortex shedding peaks on the power spectra were slightly stronger than the amplitudes 
associated with the power spectra of the vortex shedding from the spheres.

The procedure used to exami ne the lock-on regions for the sphere was similar to that used when the cable lock-on regions were determined. The spheres were first placed at four spanwise locations along the cable; $y / d=0,-24,-32$, and -42 . The cable was vibrated in the first mode and the vibration amplitude, $a / d$, was measured by the Physitech optical head and rms meter at the position $y / d=4.0$. The hot wire probe was placed directly behind the first sphere $(y / d=0)$ at the position, $x / d=0.92$ and $z / d=3.5$. The Reynolds number was slowly increased and the lower and upper lock-on frequencies for the sphere were determined. The same procedure was followed for the spheres located at $y / d=-24,-32$, and -42 . Four more lock-on regions at different a/d values were measured when the cable was vibrated in the second mode. The possibility that the vortex shedding from the sphere could lock-on to one half the vibration frequency, as we 11 as to the vibration frequency itself was also examined.

\section{E. Sphere Region of Influence}

The interactions between the sphere and cable wakes in both a stationary and vibrating situation were studied. Of particular interest was the spanwise range of influence that the vortex shedding from the sphere had on the vortex shedding from the cable. From preliminary observations of power spectra it was decided, when the 
amplitudes of the peaks associated with the sphere and cable shedding frequencies displayed on the power spectra were equal, that this position would be considered the end of the spanwise region of influence of the sphere.

To measure the region of influence, the hot wire probe was placed at a spanwise location four cable diameters $(y / d=4.0$ ) away from the center of the sphere, which was located at $y / d=0$. The probe tip" was then placed at a height $\mathrm{x} / \mathrm{d}=0.92$ and downstream position $z / d=3.5$. The probe was traversed in smal1 $1 / 20 \mathrm{inch}(\mathrm{y} / \mathrm{d}$ $=0.11$ ) increments toward the center of the sphere. The spanivise location where the magnitudes of the sphere and cable shedding peaks were equal on the power spectrum of the filtered hot wire signal was recorded. The probe was then traversed away from the center of the sphere and the entire region of influence was established. The variation of this region of influence with increasing Reynolds number for the cases of a non-vibrating and vibrating cable-sphere combination was studied, and the variation of this spanwise region of influence with changing a/d was also studied. Four spheres were placed on the cable at the locations, $y / d=0,-24,-32,-42$. The cable was vibrated in the first mode and the vibration amplitude at $y / d=4.0$ was measured using the Physitech optical head and rms meter. The probe was again placed four diameters away from the center of the vibrating sphere $(y / d=0)$, and traversed in the spanwise direction across the sphere to determine the entire region of influence. The same procedure was followed at each of the span- 
wise positions of the three remaining spheres, $y / d=-24,-32$ and -42, which were all vibrating at different amplitudes (a/d values).

Five spheres were placed on the cable at the locations

$y / d=-32,-16,0,16,32$, and their influence on the spanwise vortex shedding characteristics of the vibrating cable in unsheared flow was investigated. It was desired to determine whether or not the same phenomena would be observed when these results were compared to those obtained without the spheres. (See the end of Section IV B. "Spanwise variation of the vortex shedding frequency from a vibrating cable at Reynolds numbers preceding and following the lock-on regi on..." )

\section{F. Spanwise Sheared F1 ow Tests}

The maximum value of the shear parameter, $\beta=d / U_{c}(d U / d y)$, that could be achieved in the wind tunnel was $\beta=0.0053$. Four different tests were performed to examine the spanwise vortex shedding characteristics of the cable wake when the cable was subjected to this sheared flow. The first of these tests examined the spanwise shedding structure at three different Reynolds numbers. The cable was then vibrated in the first and second modes, and the spanwise shedding characteristics were again observed at three Reynolds numbers. Spheres, spaced at $y / d=20$ for one test and $y / d=28$ for the other, were added to the non-vibrating cable. The effect of adding the spheres, and varying their spacing, on the shedding pattern was examined. Lastly, the cable with the spheres attached 
was vibrated in the first mode. The effects that these two spacing combinations had on the vortex shedding patterns in the cable wake were studied.

The three Reynolds number values that were used when the vortex shedding structure in the non-vibrating cable wake was studied were $\operatorname{Re}_{c}=2.6 \times 10^{3}, \operatorname{Re}_{c}=2.96 \times 10^{3}$ and $\operatorname{Re}_{c}=3.72 \times 10^{3}$. The two tests at $\operatorname{Re}_{c}=2.6 \times 10^{3}$ and $\operatorname{Re}_{c}=3.72 \times 10^{3}$ were performed during the initial first phase of the investigations, three months before the main second phase of the tests. The hot wire probe during the first phase was set at a much less than optimal position, $\mathrm{x} / \mathrm{d}=1.25$ and $\mathrm{z} / \mathrm{d}=5.5$, than was later found to be required to obtain a clear picture of the actual shedding structure. For the test at $\operatorname{Re}_{c}=2.96 \times 10^{3}$, the hot wire probe was positioned at the optima 1 height, $x / d=0.92$, and downstrean 1 ocation, $z / d=3.5$. The procedure used to measure the values of vortex shedding along the span of the cable, for both the first and second phases of the investigation was the same. The probe was placed at the spanwise 1oction, $y / d=-42.7$ (first phase) or $y / d=-48$. The entire span of the cable, $\mathrm{y} / \mathrm{d}=-42.7$ to $\mathrm{y} / \mathrm{d}=40$ (first phase) or $\mathrm{y} / \mathrm{d}=-48$ to $\mathrm{y} / \mathrm{d}=48$, was traversed in increments of $\mathrm{y} / \mathrm{d}=2.7$ (first phase) or $y / d=2.0$. At each increment, a power spectrum of the filtered hot wire signal was calculated by the FFT and displayed on the terminal screen. Using the peak search command, the dominant vortex shedding frequencies at each location were identified and also printed on the terminal screen. A photograph (hard copy) of the 
terminal screen was taken to record the power spectrum and vortex shedding frequency values at each position.

For the second series of tests, the cable was vibrated in the first mode at the two lower Reynolds numbers, $\operatorname{Re}_{c}=2.6 \times 10^{3}$ and $\operatorname{Re}_{c}=2.96 \times 10^{3}$, and in the second mode at $\operatorname{Re}_{c}=3.72 \times 10^{3}$. These Reynolds number values had been previously chosen such that the cable vortex shedding would lodk on to the cable vibration frequency over the central portion of the cable span. The amplitudes of vibration for each of these tests were measured using the Physitech optical head and rms meter, and recorded. The span of the cable, from $\mathrm{y} / \mathrm{d}=-42.7$ to $\mathrm{y} / \mathrm{d}=40$ (first phase) or from $\mathrm{y} / \mathrm{d}=-48$ to $\mathrm{y} / \mathrm{d}=40$, was traversed in increments of $y / d=2.7$ (first phase) or $y / d=2.0$. At each increment, the power spectrum and shedding frequency values were calculated and photographed as in the previous set of nonvibrating cable tests.

When the spheres were added to the non-vibrating cable, two different spacing configurations were tested. In the first configuration, five spheres were placed at the spanise locations, $y / d=-40,-20,0,20,40$. Three spheres were placed at locations, $\mathrm{y} / \mathrm{d}=-28,0,28$, in the second configuration. Each of these configurations were tested at the Reynolds number, $\operatorname{Re}_{c}=2.96 \times 10^{3}$. The probe was placed at the optima 1 position, $x / d=0.92$ and $z / d=3.5$. The span of the cable, from $y / d=-48$ to $y / d=48$, was traversed in increments of $y / d=2.0$. At each increment, the powe $r$ 
spectrum and vortex shedding frequency values were calculated and photographed.

The final two tests in this sequence of four were to vibrate the cable in the first mode with each of the two sphere configurations on the cable. The amplitude of the first mode vibration was measured using the Physitech and rms meter. The Reynolds number for the first test (five spheres placed $y / d=20$ apart) was, $\operatorname{Re}_{c}=2.96 \times 10^{3}$. When the second configuration was tested (three spheres placed $y / d=28$ apart) the Reynolds number was slightly different, $\operatorname{Re}_{c}=2.93 \times 10^{3}$. The cable span, from $\mathrm{y} / \mathrm{d}=-48$ to $\mathrm{y} / \mathrm{d}=40$, was traversed in increments of $y / d=2.0$. Again, the powe $r$ spectra and frequency value were displayed on the terminal screen and photographed.

Throughout the second phase of investigations, those tests performed at $\operatorname{Re}_{c}=2.96 \times 10^{3}$ and $\operatorname{Re}_{c}=2.93 \times 10^{3}$, it was desired to keep the Reynolds number, vibration amplitudes, and cable vibration frequencies as close to a constant value as possible. This would allow direct comarisons of the data obtained in all six of the tests. From these comparisons, it was hoped that some light would be shed on the effects associated with placing spheres on a vibrating cable in a sheared flow. 
V. Results and Discussion

\section{A. Cable Mode Shapes}

The experiment had been designed [II.G] in order to measure the flow properties in the near wake of a flexible cable forced to vibrate in the first and second modes. The nondimensional first mode shape is shown in Figure 12. The absolute value of a/amax (percent), where $a$ is the measured local spanwise vibration amplitude and $a_{\max }$ is the maximum value of the measured amplitude (antinode), is plotted against the spanwise location (y/d) along the cable. The shape was invariant with vibration amplitude, $a_{\max } / d$, evidenced by the collapse of the second phase $\left(a_{\max } / d=21.0\right.$ percent and 26.5 percent) and first phase $\left(a_{\max } / d=37\right.$ percent) data points onto a single curve. The maximum deviation of the data points from the mean curve was \pm 3 percent. It should be noted that most of the data points at $a_{\max } / d=26.5$ percent and 21.0 percent plotted on top of each other, and only those few points (open squares) with significantly different values were plotted separately. The measured values of the vibration amplitudes (a) along the span of the cable were accurate to within \pm 4 percent (accuracy of the Physitech rms meter setup).

During the first phase of the investigations, a vibration wedge was used to measure the spanwise vibration amplitude, as we 11 as the Physitech and rms meter arrangement. The two values were found to correspond to within \pm 6 percent. Reading the vibration wedge accurately is a very difficult task, and this crude comparison was performed for the purpose of completeness only. 
The first and second phase second mode shapes are presented in Figure 13a, b respectively. The motor was positioned at $y / d=43.1$ during the second phase tests, and at $y / d=40$ for the first phase tests. This difference in motor position had an influence on the location of the central node during the two tests. The node was located at $\mathrm{y} / \mathrm{d}=12$ for the second phase tests, so that the mode shape was approximately a $3 / 4$ sine wave. The node was located at $y / d=0$ for the first phase tests, and the shape was characterized by a full sine wave. The second mode shape was also invariant with $a_{\max } / d$

\section{B. Lock-on Phenomena}

In this section, the near wake vortex shedding from a stationary and forced-vibrating cable and sphere cable combination was discussed. Particular emphasis was placed on synchronization (1ockon) related phenomena.

The frequency boundaries of the cable lodk-on regions $\left(a_{c v} / a_{c s v} \geq 20.0\right)$ are plotted in Figure 14 as a function of increasing vibration amplitude a/d, for the amplitude range 0.02 to 0.32. It was desired to obtain a mathematical expression, similar to those obtained by Stansby [44] for the lock-on range of a vibrating cylinder, for the bounds of cable lock-on. Linear regression by the method of least squares was used to fit a line through the data points describing the lower and upper boundaries of the lock-on 
region. The linear fit through the lower boundary

$$
\begin{aligned}
& \left(\mathrm{S}_{\mathrm{csv}} / \mathrm{S}_{\mathrm{cv}}=\mathrm{f}_{\mathrm{csv}} / \mathrm{f}_{\mathrm{cv}}<1.0\right) \text { data is } \\
& \mathrm{f}_{\mathrm{csv}} / \mathrm{f}_{\mathrm{cv}}=0.97(1.0-0.69 \mathrm{a} / \mathrm{d}),
\end{aligned}
$$

with $\mathrm{r}^{2}=0.94$, and the equation defining the upper boundary is

$$
\mathrm{f}_{\mathrm{csv}} / \mathrm{f}_{\mathrm{cv}}=1.03(1.0+1.14 \mathrm{a} / \mathrm{d})
$$

with $\mathrm{r}^{2}=0.98$. The accuracy of this linear fit is described by the value of $r^{2}$, where $r^{2}=1.0$ defines a perfect linear fit. Both the second phase (closed squares) and first phase (open circles) results exhibited no significant amount of scatter around the least squares fit, which demonstrated the reproducibility of the results over a long time period. The numerical values of the frequencies defining the upper and lower boundaries of the lock-on regions could be measured accurately to within \pm 1.5 percent. The largest error was introduced by the uncertainty in the vibration amplitude a/d measurements: \pm 4 percent for the second phase tests and \pm 10 percent for the first phase tests.

The frequency range over which the vortex shedding locked-on to the cable vibration increased proportionally with the amplitude, a finding that was consistent with Koopmann's [25] and Stansby's [44] results. Koopmann's data fell within the region enclosed by the current data whereas Stansby's data showed a larger lock-on region. 
The differences in the length of the lock-on regions could be attributed to the differences in the near wake of the vibrating cylinder and helically wound cable, and differences in the measurement procedures and criteria used to define the lock-on region. The shedding remained locked-on to the cable vibration significantly longer at the upper boundary. In the unforced situation, as the flow speed past the cable was increased, the vortex shedding self excited the cable into resonance only when the natural frequency was equal to or less than the shedding frequency $\left(\mathrm{f}_{\mathrm{cso}} / \mathrm{f}_{\mathrm{cv}}\right.$ $>$ 1). This synchronization then persisted as the flow speed was increased until the vortex shedding was no longer able to naturally excite one of the natural modes. In unforced synchronized vibrations, there was no substantial lower lock-on region. The behavior of the vortex shedding behind a forced-vibrating cable was therefore very similar to that exhibited during natural synchronization. In the present results, the frequency boundaries of the lock-on region were insensitive to whether the wind speed was ascending or descending through the region.

Lock-on phenomenon can be further examined by plotting the reduced velocity, $U_{r}$, at the boundaries of the lock-on region vs. the vibration amplitude. The reduced velocity was a nondimensional quantity that was frequently used when discussing self-excited vibrations at a natural structural frequency. It was defined as $\mathrm{U}_{\mathrm{r}}=\mathrm{U}_{\mathrm{c}} / \mathrm{f}_{\mathrm{cv}} \mathrm{d}=\left(\mathrm{f}_{\mathrm{cso}} / \mathrm{f}_{\mathrm{cv}}\right) \mathrm{U}_{\mathrm{c}} / \mathrm{f}_{\mathrm{cso}} \mathrm{d}=\left(\mathrm{f}_{\mathrm{cso}} / \mathrm{f}_{\mathrm{cv}}\right) \mathrm{St}^{-1}$. $\mathrm{U}_{\mathrm{r}}$ * was the reduced velocity at perfect synchronization $\left(\mathrm{f}_{\mathrm{csv}}=\mathrm{f}_{\mathrm{cv}}\right.$ ). 
For self-excited oscillations of a structure, the unsteady displacement amplitude first built up as $U_{r}$ approaches $U_{r}$ * toward a maximum amplitude which usually occurred at $\mathrm{f}_{\mathrm{cso}} / \mathrm{f}_{\mathrm{cv}}=1.2$, and then decreased as the upper 1 imit of the synchronization range was reached $\left(U_{r}>U_{r}^{*}\right)$. The excitation $r$ ange of these self induced oscillations in air was $4.5<U_{r}<7.5 \quad[20]$ and the maximum displacement amplitudes occurred in the range $5.0<U_{r}<6.7[20]$.

The reduced velocities at the upper and lower boundaries of the cable lock-on region, as a function of forced vibration amplitude a/d, were plotted in Figure 15 for values of a/d up to 0.322 . Perfect synchronization occurred at the critical reduced velocity value $U_{r}^{*}=5.50 \pm 2.5$ percent. The corresponding Reynolds number and velocity values were $\operatorname{Re}_{c}=2.93 \times 10^{3}$ and $U_{c}=12.98 \mathrm{ft} / \mathrm{sec}$ \pm 2.0 percent. At this critical reduced velocity, a discontinuous change in the time averaged and fluctuating forces on an oscillating cylinder occurred [50]. In Figure 15, the cable vortex shedding locked-on to the forced vibration frequency at a constant reduced velocity over the lower boundary, $U_{r}<U_{r}^{*}$. This mean value, $U_{r}=5.10 \pm 3$ percent, was 7.3 percent less than the critical reduced velocity. The upper boundary of the lock-on region increased linearly with a/d, a phenomenon that was expected to occur during both forced and unforced synchronization. In natural synchronization, when $U_{r}<U_{r}$ the cable could be self-excited because the periodic force did not have a component in phase with the oscillatory velocity of the cable. When $U_{r} \approx U_{r}{ }^{*}$, the vortex shedding and 
natural vibration frequencies perfectly synchronized (are in phase) and the cable exhibited the maximum self-excited resonance vibrations if the system damping was sufficiently sma 11. This frequency capture (resonant in-phase vibrations) then continued until the upper boundary of the lock-on region was reached. At this value of $U_{r}$, the resonant amplitudes died out completely and the two frequencies no longer acted in unison. Linear regression by the method of least squares was used to obtain an equation,

$$
U_{r}=5.76(1.0+0.86 \mathrm{a} / \mathrm{d})
$$

with $\mathrm{r}^{2}=0.988$, to describe the upper boundary of the lodk-on region.

A combined analysis of Figures 14 and 15 revealed that the frequency at which the vortex shedding initially locked-on to the forced cable vibration frequency was the variable that was changing with amplitude. It would be instructive to plot the Strouhal number (St ${ }_{c}=\mathrm{f}_{\mathrm{csv}} \mathrm{d} / \mathrm{U}_{\mathrm{c}}$ ) values at the upper and lower boundaries of the lock-on region as a function of increasing vibration amplitude a/d, to examine how the frequency changed. Such a plot is presented in Figure 16. For vibration amplitudes below 11 percent, the data exhibited no substantial change. The numerical values of the lower bound Strouhal numbers were consistently less than their upper bound counterparts, and both values were less than the numerical value found in uniform flow about the stationary cable. This result was 
consistent with both Griffin's [15] and Koopmann's [25] observations pertaining to vibration amplitude-related changes. They both noted that for forced vibration amplitudes less than 10 percent of a cylinder diameter, no measurable increase in the correlation or coherence of the vortex shedding along the cylinder span could be observed. Above 10 percent, there was a measurable increase in the spanwise correlation or coherence of the shedding.

For amplitudes greater than 11 percent, the Strouhal numbers defining the lower boundary of the lodk-on region decreased linearly with increasing $a / d$. The least square fit for the data was

$$
S t_{c}=0.198^{\prime}(1.0-0.835 \mathrm{a} / \mathrm{d})
$$

This reduction of Strouhal number, with increasing a/d, at the lowe $\mathrm{r}$ boundary of the lock-on region had been observed by Woo et a1 [48] in their experimental studies, and by Sarpkaya $[41,42]$ in his numerical analyses. In the synchronization region, the vibration caused the vortex formation length $\mathrm{L}_{\mathrm{f}}$ to be reduced considerably, i.e., $\mathrm{L}_{\mathrm{f}}$ varied inversely with a/d $[9,15,16]$. Accompanying this decrease in $\mathrm{L}_{\mathrm{f}}$, with increasing $\mathrm{a} / \mathrm{d}$, was an increase in the vortex strength [9,21,22]. Both Davies [9] and Griffin and Ramberg [21,22] noted that the growing vortex on a vibrating cylinder rolled up closer to the cylinder base and was stronger than that for a stationary cylinder. The vortices were shed at a lower Strouhal number because of the increased strength and period of formation. During 
synchronization, there was a constant period of time available for the vortices to form. The vortex strength was increased because each growing vortex was fed ciralation over a longer constant period of time. Sarpkaya and Shoaff [42] numerically showed that as the strength of the vortices in the near wake of a vibrating cylinder was increased, the Strouhal number decreased proportionally.

At the upper lock-on limit, the Strouhal number jumped to a constant value (independent of a/d) slightly less (2.5 percent) than that found in uniform flow about a stationary cable flow. This result was consistent with one of the observations listed by Sarpkaya [41] in his summary of the primary consequences of synchronized shedding. He stated that at the end of the lock-on range the vortex shedding frequency jumped to a value governed by the Strouhal relationship.

It was desired to examine the range of Reynolds numbers over which the vibration had significant influence on the Strouhal number. Figure 17 presents a plot of the centerline Strouhal number variation with Reynolds number in the near wake of a stationary $(a / d=0.0)$ and vibrating $(a / d=0.24)$ cable. The results spaned the entire lock-on region. The stationary cylinder Strouhal number values decreased by 2.5 percent over the Reynolds number range examined. Adjacent to the lower boundary of the lock-on region $\left(\operatorname{Re}_{c}<2.75 \times 10^{3}\right)$, the Strouhal numbers associated with the vibrating cable were centered around a constant value of $S t_{c}=0.157$ 士3. 2 percent. It will be shown later in this chapter (Figure 30), 
that the formation length was also substantially reduced in this region adjacent to the lock-on range. The vortices were shed at a lower Strouhal number because of the increased vortex strength which accompanied the decrease in the formation length. It was also noted [41] that in the regions adjacent to the lock-on range, the vortex shedding was intermittent between forced and unforced. This also contributed to the strength of the vortices, thereby lowering the Strouhal number. At the upper boundary of the lock-on region $\left(\operatorname{Re}>3.85 \times 10^{3}\right)$, the Strouhal number jumped to within 3.6 percent of the non-vibrating Strouhal number, a result that was consistent with those found in Figure 16 and in Sarpkaya's [41] summary - The Strouhal number was slightly less than that value found in stationary flow due to the intermittency of forced and unforced shedding at the upper boundary. This probably caused the vortices to be slightly stronger and the Strouhal number slightly smaller. The formation lengths in the adjacent region (Figure 30) were also measurably less than those behind the stationary cable, another indication that the vortices were stronger. At still higher Reynolds numbers, the vibrating and stationary curves merged, and the vibration no longer had an appreciable influence on the vortex strength, formation length and shedding frequency .

When Ramberg and Griffin [36] examined the wake behind their vibrating cable, they were able to define three distinct flow regimes (these are discussed in detail in IV. D) along the vibrating cable span. The spanwise power spectra in Figure 18 showed how the vortex 
shedding and cable vibration frequencies interacted during lod-on. The cable was vibrated in the first phase second mode with a maximum vibration amplitude of 11.7 percent. The flow Reynolds number was $3.83 \times 10^{3}$. All three distinct flow regions were present along the cable span. The length and position of each of the three regions were defined by the presence of three distinct types of power spectra. The spectrum at the cable node $(y / d=0)$ closely resembled that found in the case of a stationary body, where the shedding fluctuations in the near wake occurred principally at the Strouhal frequency. The spectra between $y / d=-16.0$ and -5.3 and $y / d=5.3$ and 16.0 , and those spectra located at $y / d=42.7,37.3$ and 40 , we re representative of the transition region where both the Strouhal and cable vibration $\left(f_{c v}=82.5 \mathrm{~Hz}\right.$ ) frequencies were present. In these regions, the component of the vibration frequency only contributed significantly to the motion. The spectra centered around the two antinodes, $\mathrm{y} / \mathrm{d}=-37.3$ to -16.0 and $\mathrm{y} / \mathrm{d}=21.3$ to 32 , were dominated by the vibration frequency (i.e., the vortex shedding was lodked-on to the cable vibration). The spanwise shedding in this region was highly correlated.

It was desired to see what influence the variation in spanwise vibration amplitude (mode shape) would have on the vortex shedding along the cable, at Reynolds numbers below and above the lock-on regions. Figures 19-21 present plots of the spanwise shedding frequencies at various Reynolds numbers. The results at Reynolds numbers below the lock-on region (Figures 19a, b and 20a) already had 
been shown to have been influenced by the vibration (i.e., shorter formation length, stronger vortices, and lower Strouhal number). The three figures clearly demonstrated that the vortex shedding frequency (Strouha1 number) decreased (by as much as 15 percent) with increasing a/d along the cable span. The minimum value of the shedding frequency in each of the figures could be found at the cable antinode, where the vibration $\left(a_{\max } / d\right)$ was the largest. Both Griffin $[15,16]$ and Davies [10] had shown that the vortex formation length decreased with increasing $a / d$, and that the resulting vortex strength increased. In the present results, the increase in a/d along the span suggested a corresponding decrease in $L_{f}$ and an increase in vortex strength. The increase in vortex strength decreased the St rouhal number a long the span.

Figures $20 \mathrm{~b}$ and $21 \mathrm{a}, \mathrm{b}, \mathrm{c}$ show the results obtained for Reynolds numbers above the lock-on region. The four figures clearly illustrated the general amplitude dependent phenomena at these Reynolds numbers. The shedding frequency (Strouhal number) increased proportionally with vibration amplitude, a/d. This was directly opposite the pattern found for Reynolds numbers below the lock-on region. These increases were all sma 11, less than 5 percent. The maximum value of frequency occurred at the antinode, where the vibration amplitude was the largest $\left(a_{\max } / d\right)$. The formation lengths were found to be insignificantly affected (Figures 27 to 30 ) by the vibration at these Reynolds numbers. The vortex shedding frequencies were greater than, and not locked-on to, the vibration frequency. 
The vortices were no longer shed at the position of maximum amplitude, as they were during synchronization. They were now shed at random positions. The wake oscillated sinusoidally because of the vibration, and the magnitude of these oscillations depended on the spanise location (i.e., vibration amplitude a/d) along the cable. This combination of increased wake oscillations and variation of the position at which the vortices were shed with increasing a/d, probably resulted in the increased destruction and cancellation of vorticity in the shed vortices. The accomanying decrease in vortex strength with increasing a/d then caused the Strouhal number to increase.

An other major objective of the present study was to observe the changes in the near wake spanwise vortex shedding patterns that were generated by placing spherical bluff body shapes on the stationary and vibrating cable in a linear shear flow. Preliminary to any shear flow tests, the Strouhal number and lock-on regions associated with an individual sphere-cable combination had to be determined. The mean Strouhal number value of the sphere-cable combination was found to be constant throughout the Reynolds number range (based on sphere diame ter) $6.57 \times 10^{3} \leq \operatorname{Re} \leq 24.17 \times 10^{3}$, at a value $S t_{s}=0.296$ \pm 0.004 . The increased bluntness of the sphere-cable combination caused the Strouhal number to be less than that associated with an isolated sphere, for which St ${ }_{S}=0.37$ (Hoerner [24]). This value was significantly higher than those measured by Achenbach [1] and by $\mathrm{PaO}$ and Kao [29], but fell well within the data scatter of all the 
tabulated measurements.

The frequency boundaries, $\mathrm{f}_{\mathrm{ssv}} / \mathrm{f}_{\mathrm{cv}}$, defining the lock-on regions of the sphere-cable combination are shown in Figure 22. The maximum vibration amplitude, based on sphere diameter, was $a / d_{s}=8.0$ percent. The least-squares linear fit through the data points defining the lower end of the lock-on region $\left(f_{s s v} / f_{c v}<1.0\right)$ was

$$
\mathrm{f}_{\mathrm{ssv}} / \mathrm{f}_{\mathrm{cv}}=0.95\left(1.0-5.22 \mathrm{a} / \mathrm{d}_{\mathrm{s}}\right)
$$

with $\mathrm{r}^{2}=0.94$. The linear fit through the data points defining the upper boundary $\left(\mathrm{f}_{\mathrm{ssv}} / \mathrm{f}_{\mathrm{cv}}>1.0\right)$ was

$$
\mathrm{f}_{\mathrm{s} \mathrm{sv}} / \mathrm{f}_{\mathrm{cv}}=0.96\left(1.0+4.68 \mathrm{a} / \mathrm{d}_{\mathrm{s}}\right)
$$

with $\mathrm{r}^{2}=0.96$. Unlike the cable lock-on region (Figure 14), where the lower lock-on region was substantially smaller than the upper region, the lower sphere-cable lock-on region was measurably wider than the upper region. Also, the total widths of the sphere-cable lock-on regions were substantially larger, than their bare cable counterparts. The data points in Figure 22 were representative of the lock-on regions centered around $\mathrm{f}_{\mathrm{ssv}} / \mathrm{f}_{\mathrm{cv}}=1.0$. During the tests, it was observed that the sphere-cable combination also lockedon to a submultiple $\left(f_{S s v} / f_{C V}=1 / 2\right)$ of the vibration frequency over a wide range. There was a very sma 11 frequency range separating the upper boundary of the lock-on region centered around $\mathrm{f}_{\mathrm{ssv}} / \mathrm{f}_{\mathrm{cv}}=1 / 2$ 
and that centered around $\mathrm{f}_{\mathrm{ssv}} / \mathrm{f}_{\mathrm{cv}}=1.0$. For large amplitudes of vibration, one would expect the two regions to merge, thereby creating a single very wide lock-on region. Because of this interaction between the two regions, the lower boundary was extended such that it became larger than the upper boundary.

Figure 23 presents a plot of the reduced velocity, $U_{r}=U_{c} / f_{c v} d_{s}$, at the boundaries of the sphere-cable lock-on regions as a function of the vibration amplitude $a / d_{s}$. The mean value of the critical reduced velocity associated with the sphere-cable combination was $U_{r}^{*}=3.39 \pm 0.23$. This value was different than the value characterizing cable lock-on, $\mathrm{U}_{\mathrm{r}}{ }^{*}=5.50$, mainly because of the different types of vortex shedding patterns associated with the two different bluff body shapes. The maximum amplitude at which the sphere-cable combination was vibrated was less than 10 percent. For this reason the data could not be comared further to the cable lockon data because of the uncertainty associated with the cable lock-on properties below 10 percent. The least squares fit through the data points defining the lower boundary of the sphere-cable lock-on region was

$$
U_{r}=3.21\left(1.0-3.63 \mathrm{a} / \mathrm{d}_{\mathrm{s}}\right)
$$

with $\mathrm{r}^{2}=0.90$, and the equation defining the upper boundary was

$$
\mathrm{U}_{\mathrm{r}}=3.49\left(1.0+3.02 \mathrm{a} / \mathrm{d}_{\mathrm{s}}\right)
$$


with $\mathrm{r}^{2}=0.77$. The width of the sphere-cable lock-on region increased in proportion to $a / d_{s}$, just as the width of the cable lockon region had.

The interactions between the sphere and cable vortex wakes behind both a stationary and vibrating sphere-cable combination were studied to examine the spanwise range over which the sphere vortex wake influenced the cable vortex wake. The entire range of influence for the non-vibrating combination was 5.9 cable diameters, and for the vibrating combination the range was 5.8 cable diameters. The length of this range was found to be Reynolds number independent (in the Re range examined), and vibration amplitude independent (for values $a / d \leq 0.26)$

It was desired to determine whether or not any type of stabilizing influence, on the already amplitude-dependent shedding structure (see Figures 19a and 20a), would be generated by placing five spheres at equidistant locations on the cable span. The five spheres were placed at $\mathrm{y} / \mathrm{d}=-32.0,-16.0,0.0,16.0,32.0$ and the cable was vibrated in the first mode with an amplitude a/d $=0.242$. The cable shedding was not locked-on to the vibration at $\operatorname{Re}_{c}=$ $2.23 \times 10^{3}$; however the spheres were near to being locked-on to a submultiple $\left(f_{s s v} / f_{c v}=1 / 3\right)$ of the vibration. The spanwise variation of the Strouhal number along the vibrating cable, with the five spheres attached, is shown in Figure 24. Compared to Figures $19 a$ and $20 a$, where the vortex shedding decreased gradually as a/d increased, the vortex shedding was generally forced into cells of 
constant frequency between the spheres. Except for the end cell which was dominated by the motor wake $(y / d=36.0$ to 40.0$)$, a11 the cells had less than a $\pm 0.5 \mathrm{~Hz}$ variation away from the mean value. The general pattern of decreasing Strouhal number with increasing a/d was still preserved within this forced cellular structure. The presence of the spheres on the cable was able to alter (stabilize the shedding into cells) the spanwise shedding frequency structure significantly when compared to the bare cable case.

\section{Formation Lengths and Wake Widths}

Typical formation length $\left(L_{f}\right)$ and wake width $\left(L_{w}\right)$ traces, obtained in the near wakes of both the stationary and vibrating cables, are shown in Figure 25. The formation lengths were measured by noting the maximum value of the filtered rms anemometer signal as the probe was traversed in the downstream (along wind) direction at the base of the cable. The wake width values were obtained by measuring the vertical distance between peak values of the filtered rms signal trace as the hot wire was traversed perpendicular to the base line, at the corresponding formation length. Most of the experimentally measured values of the formation length, shown in the next five figures, were reproducible to within \pm 4.0 percent of an average mean value, with a few extreme values differing by 8.0 percent. The reproducibility of the wake width values was slightly better than the formation lengths at \pm 3.0 percent from the mean value. In each of the next five figures (26-30), the formation 
length and wake width values are shown as functions of Reynolds number, corresponding to the five individual values of vibration amplitude tested. Changes in the formation lengths and wake widths that could be directly related to increasing Reynolds number, vibration amplitude variations, and synchronization will be discussed.

The formation length and wake width values, in the near wake of the stationary cable $(a / d=0)$, are plotted in Figure 26 for the Reynolds number range $2.0 \times 10^{3}$ to $4.2 \times 10^{4}$. It was immediately obvious that the formation length was highly Reynolds number dependent. Measurable differences in the shape of the curve and magnitudes of the values were evident when these near wake cable results were compared with the smooth circular cylinder results (Figure 1). These differences were the result of the changes in the near wake created by the helically wound cross section of the cable. Between Reynolds numbers $2.0 \times 10^{3}$ and $1.0 \times 10^{4}$, the cylinder and cable $\mathrm{L}_{\mathrm{f}}$ curves exhibited a decrease in $L_{f}$ with increasing Reynolds number. The cable values were measurably larger than the cylinder values, 14 percent at $2.0 \times 10^{3}$ and 33 percent at $1.0 \times 10^{4}$. At $\operatorname{Re}=1.0 \times$ $10^{4}$, the formation length was a minimum on both curves, with $L_{\mathrm{f}} / \mathrm{d}=$ 1.32 behind the cylinder and $\mathrm{L}_{\mathrm{f}} / \mathrm{d}=1.75$ behind the cable. The smooth cylinder values then remained constant with Reynolds number out to $\operatorname{Re}=1.5 \times 10^{5}$, at the minimum value $L_{\mathrm{f}} / \mathrm{d}=1.32$, while the cable values increased with Reynolds number, such that at $\operatorname{Re}=4.1 \times 10^{4}$ the value was $\mathrm{L}_{\mathrm{f}} / \mathrm{d}=2.10$. The helical cross section of the cable 
lengthened the vortex formation region, the same effect that roughness had [32] on the formation region.

The major similarities and/or differences that we re observed when the four sets of vibrating formation length results (Figures 27a-30a) were compared with the stationary results (Figure 26a) are discussed below.

(1) Be low the synchronization region (region centered around $\operatorname{Re}=2.9 \times 10^{3}$ ), the formation lengths associated with the vibrating cable were significantly less than the stationary cable values. This amplitude-dependent reduction was expected, based on the analysis of the results of Figure 17. The strength of the vortices was increased (1 ower Strouhal number), which suggested that the vortex formation length decreased correspondingly. At the two lowest vibration amplitudes, $a / d=9.9$ percent and 13.0 percent, the Reynolds number dependent decrease of $L_{f}$ was preserved, although the vibrating cable values were significantly smaller than the stationary ones. At the two highest vibration amplitudes, $a / d=17.6$ percent and $a / d=25.5$ percent, the Reynolds number decrease was obscured by the larger vibrationinduced changes, which made $\mathrm{L}_{\mathrm{f}}$ relatively constant before the lock-on region. This was also expected, since the vibration-induced Strouhal number was also constant with Reynolds number (Figure 17, $\mathrm{a} / \mathrm{d}=24.0$ percent) at the higher vibration amplitude.

(2) The Reynolds number around which the minimum formation length was found $\left(\operatorname{Re}=1.0 \times 10^{4}\right)$ did not change as a result of the vibration. The numerical value, $\mathrm{L}_{\mathrm{f}} / \mathrm{d}=1.70$, was also not signifi- 
cantly influenced by the addition of vibration. The five curves a 11 overlayed upon each other, with minimal data scatter, for Reynolds numbers greater than $1.0 \times 10^{4}$. The vibration had a minima 1 influence on the formation lengths in this Reynolds number region far removed from the lock-on range.

(3) In the Reynolds number range directly above the lock-on region and extending to the minimum $L_{f}$ point, the four vibrating cable curves merged into the stationary curve at Reynolds numbers ranging between $4.0 \times 10^{3}(\mathrm{a} / \mathrm{d}=9.9$ percent $)$ and $6.0 \times 10^{3}(\mathrm{a} / \mathrm{d}=25.5$ percent). These Reynolds number values marked the points at which the vibration no longer had any significant influence on the vortex formation process above the lodk-on region.

When the four vibrating cable formation length Figures (27a-30a) were comared a significant synchronization related phenomenon could be observed. A sudden increase in $\mathrm{L}_{\mathrm{f}} / \mathrm{d}$ occurred during perfect synchronization, i.e. in the region centered around $\mathrm{f}_{\mathrm{csv}} / \mathrm{f}_{\mathrm{cv}}=1.0$. This perfect synchronization point occurred at a reduced velocity, $\mathrm{U}_{\mathrm{r}}{ }^{*}=5.50$ (Figure 15), and Reynolds number $2.93 \times 10^{3}$. On the p1ots, this Reynolds number marked the center of the sudden increase in $\mathrm{L}_{\mathrm{f}} / \mathrm{d}$. Shortly after the value of $\mathrm{L}_{\mathrm{f}} / \mathrm{d}$ reached a maximum, the four vibrating curves merged into each other, as well as merging into the stationary curve. The maximum value reached by $\mathrm{L}_{\mathrm{f}} / \mathrm{d}$ was inversely proportional to the amplitude a/d, a result consistent with previous observations where $L_{f} / d$ decreased with increasing a/d $[15,16,21,22]$. 
Griffin [19] presented a plot of base pressure $C_{p b}$ and wake width $\mathrm{L}_{\mathrm{w}} / \mathrm{d}$ variations, as a function of $\mathrm{f}_{\mathrm{cv}} / \mathrm{f}_{\mathrm{cso}}$, in the synchronization region. These two properties behaved similarly to the present $\mathrm{L}_{\mathrm{f}} / \mathrm{d}$ data in the perfect synchronization region; i.e., the values were strongly affected during synchronization and returned to stationary values shortly thereafter. Other authors have noted a sudden increase in spanwise correlation of the phase of the shedding [21], fluctuating lift and steady drag forces [50], wake width [44], and phase angle between the cylinder displacement and maximum rms pressure on that side [50]. In the background section (II.C) the two modes of synchronized vortex shedding observed by Zdravkovich [50] were described. The vortices in the upper synchronization range $\left(f_{c s v} / f_{c v}>1.0\right)$ were shed when the cylinder was near its maximum amplitude on that same side of the wake, whereas in the lower synchronization region, the vortices formed on one side of the cylinder and were shed when the cylinder was close to its maximum amplitude position on the opposite side. The longer formation length in the upper synchronization region was a direct result of the increased stability of the shedding in this region.

The wake widths in the near wake of the stationary cable (Figure 26b) varied between $\mathrm{L}_{w} / \mathrm{d}=0.92$ and 1.04 , with the mean value $\mathrm{L}_{\mathrm{w}} / \mathrm{d}=0.98$. There was no observable variation in this mean value with Reynolds number. Comparing the present helical cable results with Peltzer's smooth circular cylinder results (Figure 2) revealed no significant difference in the mean value of $\mathrm{L}_{W} / d$. Peltzer's values 
we re centered around the mean value $\mathrm{L}_{\mathrm{w}} / \mathrm{d}=0.97$ and a lso we re Reynolds number independent. The helical structure had no measurable influence on the wake widths. When the vibrating cable wake width plots (Figures $27 \mathrm{~b}$ to $30 \mathrm{~b}$ ) were compared to the stationary plot, a general increase in $\mathrm{L}_{\mathrm{w}} / \mathrm{d}$ due to the vibration could be observed. However; due to the considerable amount of scatter in the vibrating cable wake width data, no other significant conclusions regarding vibration related phenomena could be drawn.

\section{Sheared Flow Results}

Figures 31 thru 50 present twenty pairs of results dealing with the spanwise vortex street wake behind the stationary and vibrating cable in a linear shear flow $(\beta=0.0053)$, with and without attached bluff bodies. The first figure in each pair contains the power spectra of the fluctuating hot wire signal at various equidistant spanwise locations ( $y / d$ values) in the cable wake. The vortex shedding and/or vibration frequencies appear as high energy peaks in the spectra. The second figure in the pair shows the power spectra data plotted as a Strouhal number based on the centerline velocity $\left(S t_{c}=f_{c s v} d / U_{c}\right)$ versus the spanwise position $y / d$. These St $c$ vs. $y / d$ plots are presented to elucidate the spanwise cellular structure in shedding frequency, if and where it existed. A single closed circle on these plots represents a narrow band shedding frequency peak on the power spectra. A circle connected to a bar represents a high energy narrow band shedding peak with a lower energy wide band to either side 
of the peak, and a bar represents a wide broadbanded peak with no dominant high energy shedding peak present.

Figures 31 to 38 are first phase results and Figures 39 to 50 contain the second phase results. The main difference between the two sets of results was the location of the hot wire probe in the wake. During the first phase tests, the probe was located farther downstream of, and vertically higher than, the optimal position that was found (during the second phase tests) to provide the strongest and clearest measure of the shedding frequency in the power spectra. Because of this, any fluctuating cell boundaries were allowed a longer time to interact, and the resulting cellular structure (if it existed) was poorly defined. Normally, the marker between two cells of different frequencies would be a large discontinuous jump in frequency. The apparent gradual decrease in frequency between cells was a direct result of the fluctuating cell boundaries. Mair and Stansby [26] pointed out in their results that discontinuous jumps of frequency between cells disappeared in the tine-averaged results because of the fluctuating cell boundaries. The second phase results were not as significantly affected by the presence of these fluctuating cell boundaries. A discrete cell of constant shedding frequency in these plots was indicated by a spanwise y/d range over which the Strouhal number remains constant.

The power spectra showing the spanwise variation of the vortex shedding frequency in the near wake of the stationary cable, at a Reynolds number $2.6 \times 10^{3}$, are shown in Figure 31 . The corresponding 
spanwise Strouhal number plot follows in Figure 32. There was a definite tendency for the vortex shedding to form into cells along the entire cable span, even though the discontinuous jump in Strouhal number that should mark the division between the cells was obscured by the fluctuating cell boundaries. Including the two end cells, a total of approximately ten cells were present along the cable span (from $\mathrm{y} / \mathrm{d}=-42.7$ to $\left.\mathrm{y} / \mathrm{d}_{\mathrm{c}}=40.0\right)$, with each cell separated by a change in Strouhal number $\Delta S t_{c} \approx 0.007$. The high velocity cell was centered around $S t_{c}=0.190$ and the 1 ow velocity cell centered around $S t_{c}=0.1265$, such that the total change across the span was $\Delta S t_{c}=0.0635$ The average length of each cell was approximately 9.0 diame ters.

The power spectra along the span of the vibrating cable, at $\operatorname{Re}_{c}=2.6 \times 10^{3}$, are shown in Figure 33. At this Reynolds number, it was expected that the vortex shedding would lock-on to the cable vibration over the central portion of the cable span. The maximum first mode vibration amplitude (at the node) was $a / d=0.233$. A clearly defined spanwise cellular structure in the shedding frequency was seen in the spectra, even with the compressed frequency scale. The spanwise power spectra dramatically illustrated the three distinct flow regimes, associated with cable lodking-on, that Ramberg and Griffin [36] defined. The power spectra in the ranges adjacent to the ends of the cable (nodes), $-42.7 \leq y / d \leq-34.7$ and $37.3 \leq y / d \leq 40.0$, were representative of the flow past a stationary body. In these nodal regions, as well as in flow about a stationary cable, only the 
vortex shedding frequency was present in the power spectra. The power spectra in the areas between the nodal and locked-on regions, $-32.0 \leq \mathrm{y} / \mathrm{d} \leq 5.3$ and $29.3 \leq \mathrm{y} / \mathrm{d} \leq 34.7$, illustrated the behavior of the magnitude (strength) of the vortex shedding and cable vibration frequency peaks in the transition region between unlocked and synchronized vortex shedding. The growth (or decline) of the vibration peak as the shedding frequency approached (broke away) from the synchronization range was clearly demonstrated. In the synchronized region, centered near the cable antinode $-5.0 \leq \mathrm{y} / \mathrm{d} \leq 29.0$, the spanwise power spectra contained a sharp, high energy peak at the synchronization frequency. The spanwise shedding was highly correlated in this region.

When the spanwise Strouhal number plot of the power spectra in the wake of the vibrating cable at $\operatorname{Re}_{c}=2.6 \times 10^{3}$ (Figure 34), was compared with the stationary cable Strouhal plot (Figure 32), the stabilizing effect that the synchronized vibration had on the cellular structure in general was noticed. St ansby [44] and Woo et a1 [48] also noted this stabilization. The locked-on region was 34 diameters long, and the average cell length of the unforced cells was 13.5 diameters, significantly greater than the average length of 9.0 diameters found along the non-vibrating cable span. The high velocity cell was centered around the Strouhal number $S t_{c}=0.188$ and the 1 ow velocity cell centered around $S t_{c}=0.129$. The total change in Strouhal number across the span was $\Delta S t_{c}=0.059,7.1$ percent less than the total change across the stationary cable (Figure 32). 
The Reynolds number was increased to $3.72 \times 10^{3}$, and the corresponding power spectra associated with the stationary cable span were shown in Figure 35. It was expected that the cell lengths at this higher Reynolds number would be slightly shorter than those found at the lower Reynolds number $\left(2.6 \times 10^{3}\right)$, because of the shorter formation length. Although some tendency towards a cellular structure was seen in the spanwise Strouhal number plot (Figure 36), no conclusive observations regarding average cell lengths, number of cells, or average change in Strouhal number between cells, could be drawn. The less than optimal probe position and fluctuating cell boundaries combined to obscure the true near wake shedding pattern. There could be at least 12 cells present along the span, with the average separation between the cells $\Delta S t_{c} \approx 0.006$. The high velocity cell was centered around $S t_{c}=0.213$ while the low velocity cell occurred at St ${ }_{c}=0.146$, so that the total change in Strouhal number across the span was $\Delta S t_{c}=0.067$, slightly larger than the range $\Delta S t_{c}=0.0635$ at $\operatorname{Re}_{c}=2.6 \times 10^{3}$. The average cell length was approximately 7.5 diameters, slightly smaller than the 9.0 diameter ave rage length at $\operatorname{Re}_{c}=2.6 \times 10^{3}$.

The cable was then forced to oscillate in the first phase second mode shape at $\operatorname{Re}_{c}=3.72 \times 10^{3}$. The vortex shedding was expected to lodk-on to the vibration around the low velocity antinode. The maximum vibration amplitude at the antinode was $\mathrm{a} / \mathrm{d}_{\mathrm{c}}=11.7$ percent. The power spectra (Figure 37) again illustrated the three distinct regions associated with synchronization, i.e. unforced shedding around 
a node at $\mathrm{y} / \mathrm{d}=0$, synchronized shedding around an antinode $\mathrm{y} / \mathrm{d}=$ -26.0 , and shedding in the region between the node and antinode. The spanwise shedding structure (Figure 38) was influenced in a more complicated manner by the second mode vibration, than by the first mode vibration. There were six unforced cells of average length 8.4 diameters adjacent to the 21.0 diameter locked-on ce11. The unforced cells were separated by a jump in Strouhal number $\Delta S t_{C} \approx 0.007$, and the total change in Strouhal number across the span was $\Delta S t_{c}=0.065$, 3 percent less than the non-vibrating range. The synchronized vibration had again stabilized the spanvise cellular shedding structure in the cable wake, as it had in Figure 34. The four sets of first phase results have now been analyzed. Wi th these observations in mind, a more comprehensive analysis of the second phase results will be undertaken.

The six pairs of second phase vortex shedding results will be analyzed in the following order:

1). Figures 39 and 40; stationary cable at a Reynolds number $2.96 \times 10^{3}$.

2). Figures 41 and 42; vibrating cable $a_{\max } / d=0.291$, Reynolds number $2.96 \times 10^{3}$.

3). Figures 43 and 44; stationary cable, five spheres spaced $y / d=20.0$ apart, Reynolds number $2.96 \times 10^{3}$.

4). Figures 45 and 46; vibrating cable $a_{\max } / d=0.233$, five spheres spaced $y / d=20.0$ apart, Reynolds number $2.96 \times 10^{3}$. 
5). Figures 47 and 48; stationary cable, three spheres spaced $y / d=28.0$ apart, Reynolds number $2.96 \times 10^{3}$.

6). Figures 49 and 50; vibrating cable, $a_{\max } / d=0.235$, three spheres spaced $y / d=28.0$ apart, Reynolds number $2.93 \times 10^{3}$.

The power spectra, taken at spanwise increments of $y / d=2.0$ in the near wake of the stationary cable at $\operatorname{Re}_{c}=2.96 \times 10^{3}$, are displayed in Figure 39. A definite spanwise cellular structure was evident, where the frequency peaks within each of these cells was generally sharp and narrow-banded. At the boundaries between some cells, the spectra were broad-banded with no dominant shedding frequency or they exhibited the two distinct frequencies associated with each individual ce11. The fluctuating cell boundaries, when averaged 20 times during the two mi nute data record, allowed the contribution from both cells to be included in the spectral average. Depending on how rapidly the cell boundaries were fluctuating, the power spectrum would either be broadbanded or show the two distinct frequency peaks. Also, the frequency peaks in the spectra at the 1 ow velocity end of the cable were slightly broadbanded because of the higher turbulence leve1. This phenomenon was observed by Peltzer [32] in previous wind tunnel tests.

Not including end cells, there were 10 cells of constant Strouhal number along the cable span (Figure 40). The average length of these cells was 11.0 diameters, and they were each separated by an average discontinuous jump in Strouhal number $\Delta S t_{c}=0.0086$. The change in Strouhal number across the span $(-48.0 \leq \mathrm{y} / \mathrm{d} \leq 48.0)$ was 
$\Delta S t_{c}=0.0865$. Comparison of the change in Strouhal number from $-42.7 \leq \mathrm{y} / \mathrm{d} \leq 40.0, \Delta S t_{\mathrm{c}}=0.067$, wi th the change at $\operatorname{Re}_{\mathrm{c}}=2.6 \times 10^{3}$, $\Delta S t_{c}=0.0635$, revealed that only a sma 115.5 percent difference was observed. A comarison of the clarity of the cellular structure in Figure 40 with that of Figure 32 illustrated the importance of proper positioning of the hot wire probe in the cable wake. When the hot wire was not positioned optimally, the discontinuous jumps in Strouhal number between the cells and the actual cellular structure were not as evident as when the probe was positioned optimally.

The vibrating cable spectra, with $a_{\max } / d=0.291$ and $\operatorname{Re}_{c}=2.96 \times 10^{3}$, are shown in Figure 41. The three distinct types of spectra associated with the locking-on process were present in the figure. The shedding was locked-on to the vibration over the central portion of the cable span (Figure 42), from $-14.0 \leq y / d \leq 29.5$. The locked-on cell was 43.5 diameters 1ong. In Figure 34 (first phase test, $a_{\max } / d=0.233, \operatorname{Re}_{c}=2.6 \times 10^{3}$ ), the locked-on cel1 was 34 diameters long. The larger locked-on region in the present figure was expected because the maximum vibration amplitude was larger. The average length of the two cells, not adjacent to the end plate or motor, was 14.0 diameters, whereas the average length in Figure 34 was a comparable 13.5 diameters. The shedding was so well stabilized by the vibration that no fluctuating boundary effects were present in the figures. All the cell boundaries were marked by discontinuous jumps in Strouhal number. The change in Strouhal number across the cable span, $-48.0 \leq y / d \leq 40.0$, was $\Delta S t_{c}=0.775$ (from 0.2060 to 0.1285$)$. 
The spanise power spectra, showing the vortex shedding frequency variation along the stationary cable with five spheres at spanwise locations $\mathrm{y} / \mathrm{d}=-40.0,-20.0,0.0,20.0,40.0$, a re shown in Figure 43. The flow Reynolds number was $2.96 \times 10^{3}$. The shedding frequency of the spheres increased proportionally to the velocity across the span. Between the sphere shedding peaks, the cable vortex shedding frequency peaks were generally more broad-banded than the narrowbanded high energy peaks characteristic of the bare stationary cable (Figure 39). The highly three-dimensional turbulent wake behind the spheres broadened the shedding peaks with the increased turbulent energy it imparted to the cable wake. The three-dimensional sphere wakes totally disrupted the cellular shedding pattern normally present in the bare cable wake. Figure 44 shows that the presence of the spheres generally forced the cable shedding frequencies to form into discrete cells in each of the spanise areas between the spheres. The only slight deviation away from this forced single cellular structure was in the cell centered around $y / d=-10.0$. The spectra at $y / d=$ $-12.0,-14.0$ and -16.0 were very broad-banded, with the center of the band at a slightly lower frequency than the rest of the cell. Including the two end cells, six vortex shedding frequency cells with an average length of 14.0 diameters, separated by five sphere shedding cells $\left(\mathrm{St}_{\mathrm{c}}=\mathrm{f}_{\mathrm{ss}} \mathrm{d} / \mathrm{U}_{\mathrm{c}}\right)$ with an average length of 6.0 diameters, were located along the entire cable $\operatorname{span}(-48.0 \leq \mathrm{y} / \mathrm{d} \leq 48.0)$. The average discontinuous change in Strouhal number between each of the cable 
shedding frequency cells was $\Delta S t_{c}=0.0136$, a much larger change than $\Delta S t_{c}=0.0086$ between the bare stationary cable cells.

The cable with the five spherical bluff body shapes attached was then vibrated in the first mode $\left(a_{\max } / d=0.233\right)$. The spanwise power spectra, corresponding to flow at $\operatorname{Re}_{c}=2.96 \times 10^{3}$, are shown in Figure 45. The cable vortex shedding frequency power spectra in the regions separated by the spheres were again slightly broad-banded. All three types of spectra, characterizing the lock-on process associated with a flexible cable, were represented in the figure. In the locked-on region around $\mathrm{y} / \mathrm{d}=0.0$, the single high energy narrow-band peak characteristic of synchronous motion was present. When the frequency data were plotted in Figure 46, it was immediately apparent that the three central spheres $(y / d=-20.0,0.0,20.0)$ 1ocked-on to a submultiple of the cable vibration, $\mathrm{f}_{\mathrm{ssv}} / \mathrm{f}_{\mathrm{cv}}=1 / 2$. The presence of the spheres on the vibrating cable $\left(a_{\max } / d=0.233\right)$ had a significant effect on the length of the lock-on region. The locked-on region ext ended from $-24.0 \leq \mathrm{y} / \mathrm{d} \leq 29$ (53 diameters) compared to $-14.0 \leq \mathrm{y} / \mathrm{d} \leq 29.5$ (43.5 diameters) on the bare vibrating cable with $a_{\max } / d=0.291$, and $-5.0 \leq y / d \leq 29(34$ diameters $)$ on the bare vibrating cable with $a_{\max } / d=0.233$. The extension of the length of this lockedton region by as much as 56 percent, was very significant because it meant that the damaging undesirable effects that accompany synchronization (i.e. large amplitude oscillations, increased drag and fluctuating lift forces, highly correlated shedding, etc.) would extend over a longer portion of the cable span. The cable vortex 
shedding frequency was constant between the spheres at $y / d=40.0$ and 20.0. Between the spheres at $\mathrm{y} / \mathrm{d}=-20.0$ and -40.0 , the shedding frequency broke into two short cells separated by a slight decrease in Strouhal number. The change in Strouhal number across the span $(-48.0 \leq y / d \leq 36.0)$ of the vibrating cable with $f$ ive spheres was $\Delta S t_{c}=0.0685(0.1970$ to 0.1285$)$, compared to $\Delta S t_{c}=0.069(0.1950$ to 0.1260 ) across the stationary cable with five spheres, $\Delta S t_{c}=0.074 \quad(0.2015$ to 0.1275$)$ across the bare vibrating cable and $\Delta S t_{c}=0.0775(0.2060$ to 0.1285$)$ across the stationary cable.

The number of the spheres present on the cable was then reduced to three, and the spacing between them increased to 28.0 diameters. The resulting spanwise power spectra, corresponding to flow past the stationary cable at $\operatorname{Re}_{c}=2.96 \times 10^{3}$, are shown in Figure 47. Those cable shedding frequency peaks not directly in the regions adjacent to the spheres were narrow-banded like those associated with the bare stationary cable frequency peaks. The frequency peaks in the regions adjacent to the spheres were again broad-banded, just as they were in Figure 43. The three-dimensional turbulent wakes of the spheres were no longer able to exert influence over the entire spanwise distance between the spheres. The cable vortex shedding frequencies were not forced into discrete cells between the spheres that were spaced 28.0 diameters apart, as they had been when the spacing was 20.0 diameters. At the shear level $\beta=0.0053,20.0$ diameters was the maximum spacing that forced the cable shedding frequencies between the spheres into discrete cells. Generally, the maximum spacing that would force 
the vortex shedding frequency between these discrete bluff body shapes into identiflable cells of constant frequency should depend on the shear level $B$, the geometry of the discrete bluff body shape and the body it is mounted on, the flow Reynolds number, the vortex formation length (stability of the cells), and the stability of the flow itself (turbulence, upstream disturbances, etc. ).

The spanwise Strouhal number variation across the stationary cable, with the three attached spheres, is illustrated in Figure 48. The influence that the presence of the spheres had on the length and position of the spanwise cells of cable shedding frequency was seen when these results were compared to the stationary bare cable results (Figure 40). There were seven or eight cells of constant cable shedding frequency, which ranged in length from 2.0 to 18.0 diameters, plus the three cells which represented the sphere shedding frequency, across the cable span $(-48.0 \leq y / d \leq 48.0)$. The broad-banded ranges between a few of the cells could be attributed to a combination of fluctuating cell boundaries and three-dimensional sphere wake influences. The change in Strouhal number across the span was $\Delta S t_{c}=$ $0.0795(0.2120$ to 0.1325$)$, compared to $\Delta S t_{c}=0.069(0.1950$ to 0.126$)$ across the span of the cable with five spheres, and $\Delta S t_{c}=0.0865$ (0.2150 to 0.1285$)$ across the stationary bare cable. The average discontinuous change in Strouhal number between the vortex shedding cells on the cable with three spheres was $\Delta S t_{c}=0.0102$, as compared to $\Delta S t_{c}=0.0136$ between the cells on the cable with five spheres and $\Delta S t_{c}=0.0086$ between the cells on the bare cable. Generally, when 
the spheres were spaced at the maximum value which forced the vortices to be shed in discrete cells of constant frequency between them, the longest cells were present along the cable span with the largest discontinuous change in Strouhal number between them, and the total change in Strouhal number across the span was a minimum. As the spacing between the spheres was increased, the number of the cells increased while the average length of, and discontinuous spacing between, then decreased, and the total change in Strouhal number increased. The distribution of vortex shedding would eventually become equal to the stationary bare cable distribution.

For the sixth and final test in this series, the cable with the three spheres was vibrated $\left(a_{\max } / d=0.235\right)$ in the first mode at a Reynolds number of $2.93 \times 10^{3}$. The spanwise power spectra are shown in Figure 49. The vortex shedding from the cable was locked-on to the vibration over the entire spanwise region bounded by the spheres, $-31.0 \leq \mathrm{y} / \mathrm{d} \leq 30.0$. The spheres, located at $\mathrm{y} / \mathrm{d}=0.0$ and \pm 28.0 , were locked-on to a submutiple of the cable vibration frequency, $\mathrm{f}_{\mathrm{Ssv}} / \mathrm{f}_{\mathrm{CV}}=1 / 2$. Only the spanwise regions outside the two end spheres exhibited unforced cable vortex shedding. The length of the locked-on region, shown in Figure 50, was 61.0 diameters. This length is 15 percent greater than the 53.0 diameter locked-on region in the five sphere test (Figure 46), 40 percent larger than the locked-on range which corresponded to the bare vibrating cable with $a_{\max } / d=0.291$, and 79 percent larger than the 34.0 diameter range found on the bare cable which was vibrated at a comparable amplitude $a_{\max } / d=0.233$. 
The spanise distance between the spheres had a very significant effect on the length of the locked-on region. The total change in Strouhal number across the $\operatorname{span}(-48.0 \leq \mathrm{y} / \mathrm{d} \leq 40.0)$ was $\Delta \mathrm{St}_{\mathrm{c}}=$ 0.0705 , compared to $\Delta S t_{c}=0.0685$ across the vibrating cable with five spheres, and $\Delta S t_{c}=0.074$ along the bare vibrating cable and $\Delta S t_{c}=$ 0.0775 on the bare stationary cable. Similarly to the non-vibrating results, as the spacing between the.spheres was increased, the total change in Strouhal number across the span increased. 


\section{SUMMARY}

\section{A. Findings and Conclusions}

There were many significant results discussed in the previous section. Some corroborated previously established conclusions pertaining to stationary and vibrating circular cross section cylinders and flexible cables in uniform or sheared flow, while others had been documented for the first time. This entire study was unique in that no previous authors had studied the near wake of a stationary or vibrating cable, with attached discrete bluff bodies, in any flow situation. In addition, no studies of near wake properties of helically wound cables had been undertaken. A summary of the major results dealing with the individual and combined effects of vibration, shear, and bluff body shapes on the vortex shedding in the near wake of a marine cable is as follows:

1) The mean Strouhal number of the non-vibrating cable was constant throughout the Reynolds number range $2.0 \times 10^{3} \leq \operatorname{Re} \leq 4.2 \times$ $10^{4}$ at the value $\mathrm{St}=0.192$.

2) Locked-on vortex shedding in the near wake of the forced vibrating marine cable behaved similarly to that exhibited in the near wake of a circular cross section cable undergoing natural synchronization. The frequency range over which the vortex shedding locked-on to the cable vibration increased proportionally with the amplitude of vibration. The upper locked-on region $\left(f_{c s v} / f_{c v}>1.0\right)$ was significantly larger than the lower region. 
3) Perfect synchronization ocaurred when the critical reduced velocity was $U_{r}^{*}=5.50$. At the lower boundary of the lock-on region, the vortex shedding locked-on to the cable vibration at a constant reduced velocity which was independent of vibration amplitude a/d. The reduced velocity values that defined the upper boundary of the locked-on region increased linearly with the vibration amplitude a/d.

4) At the lower boundary of the locked-on region, the Strouhal numbers and formation lengths varied inversely with the vibration amplitude a/d, while the vortex strengths varied in direct proportion to a/d. At the upper boundary the vortex strengths, St rouhal numbers, and formation lengths in the wake of the vibrating cable were virtually amplitude independent and nearly equaled those values measured in the wake of the non-vibrating marine cable.

5) The three distinct flow regimes (flow near a cable node, antinode and in the region between the two) that were present when the vortex shedding and cable vibration frequencies interacted during lock-on, were clearly defined by the spanwise power spectra variations.

6) The mean Strouhal number of the sphere-cable combination in the sphere Reynolds number range $6.5 \times 10^{3} \leq \operatorname{Re}^{*} \leq 2.4 \times 10^{4}$ was $S t_{S}=0.296$.

7) The width of the lock-on region for the sphere-cable combination was significantly larger than the lock-on region for a bare cable. The upper and lower boundaries of the sphere-cable lodk-on region increased linearly with the vibration amplitude a/d. 
8) The helical cross section of the cable lengthened the vortex formation region and changed the Reynolds number dependent properties of $\mathrm{L}_{\mathrm{f}}$ when compared to circular cross section cable values and properties.

10) A sudden increase in the vortex formation length occurred during perfect synchronization $\left(f_{c s v} / f_{c v}=1.0, U_{r}^{*}=5.50\right)$. Short1y after the end of synchronization the $L_{f}$ values returned to stationary values. Other authors had found that the base pressure, wake width, spanwise vortex shedding phase correlation, and fluctuating 1 ift and steady drag were also strongly influenced during perfect synchronization and returned to stationary values shortly thereafter.

11) A smal1 amount of linear shear in the upstream velocity profile initiated a strong cellular vortex shedding structure in the wake of the bare cable at the Reynolds numbers considered.

12) The length of the locked-on region in sheared flow was vibration amplitude dependent.

13) The spanwise vortex shedding cellular structure was significantly stabilized by the vibration, and the unlocked cells on the vibrating cable were longer than the unforced stationary cable ce11s.

14) When the spherical bluff body shapes were added to the cable, their three-dimensional wakes significantly altered the spanwise shedding pattern normally present in the bare cable wake. A maximum separation distance $(y / d=20.0)$ was observed that would force 
the vortex shedding into discrete cells of constant frequency between the spheres.

15) The presence of the spheres along the vibrating cable span measurably increased the length of the locked-on region. The amount of increase was dependent upon the spacing between the spheres. This finding was significant because it meant that the undesirable effects that accomany synchronization were extended over a longer portion of the cable span.

\section{B. Recommendations for Further Study}

1) Measuring the width of the lock-on region as a function of vibration amplitudes greater than $a / d=0.322$ in the wake of the cable and sphere-cable combination would significantly extend the present study.

2) The dependence of the length and stability of spanwise cells of constant shedding frequency in a sheared flow on the length of the vortex formation region needs to be thoroughly studied. It is hoped that this type of study would help explain why there are or are not discrete spanwise cells at various Reynolds numbers, and also resolve some of the disagreements in the literature.

3) The discrete bluff body shapes (spheres) used in the present tests were considered massless. Adding variable masses to the bluff bodies and investigating the effects of the added mass on the spanwise lock-on regions and cellular structure would begin to realistically simulate full-scale physical situations. The present results 
could be combined with those of Vandiver and Griffin [46] to provide a basis for modifying the NCEL-developed computer code NATFREQ which was developed to calculate the natural frequencies and mode shapes of taut marine cables with large numbers of attached masses.

4) Analysis of the general dependence of the length and stability of forced and unforced spanwise vortex shedding ce11s on the attached bluff body and cable geometries, flow Reynolds number, vortex strength and formation length, and the stability of the incident flow would provide the designer with a clearer understanding of the dynamic and physical properties associated with flow around cables with bluff body shapes attached. 


\section{References}

1. Achenbach, E., "Vortex Shedding from Spheres", JFM, Vol. 62, Part 2, 1974, pp. 209-221.

2. Angrilli, F. and Cossalter, V., "Transverse oscillations of a vertical pile in waves", ASME Journal of Fluids Engineering, Vol. 104, No. 1, Mar. 1982, pp. 46-53

3. Barnett, K.M. and Cermak, J.E., "Turbulence induced changes in vortex shedding from a circular cylinder", NTIS report AD-775-885, Jan. 1974 .

4. Bendat, J.S. and Piersol, A.G., Random Data: Analysis and Measurement Procedures, Wiley-Interscience, New York, 1971.

5. Berland, G.D., "A guided tour of the fast Fourier transform", IEEE Spectrum, Jul. 1969.

6. Blevins, R.D. and Burton, T.E., "Fluid forces induced by vortex shedding", ASME Pub1ication 75-FE-10, 1975.

7. Bloor,M.S., "The transition to turbulence in the wake of a circular cylinder", JFM, Vol. 19, 1964, pp. 290-303. 
8. Bloor, M.S.and Gerrard, J.H., "Measurements on turbulent vortices in a cylinder wake", Royal Society of London Proceedings, Vol. 294, 1966, pp. 319-342.

9. Davies, M.E., "A comparison of the wake structure of a stationary and oscillating bluff body, using a conditional averaging technique", JFM, Vol. 75, Part 2, 1976, pp. 209-231.

10. Davies, M.E., "The effects of turbulent shear flow on the critical Reynolds number of a circular cylinder", National Physics Laboratory (U.K.) NPL Report Mar Sci R 151, Jan. 1976.

11. Every, M.J., King, R. and Griffin, O.M., "Hydrodynamic loads on flexible marine structures due to vortex shedding", ASME Publication 81-WA/FE-24, Nov. 1981.

12. Fischer, J.F., Jones, W.T. and King, R., "Current induced oscillations of Cognac piles during installation - prediction and measurement", Practical Experiences with flow-Induced Vibrations, E. Naudascher and D. Rockwe11 (eds.). Springer: Berlin, 1980, pp. 570-581 .

13. Gerrard, J.H., "The three-dimensional structure of the wake of a circular cylinder", JFM, Vo1. 25, Part 1, 1966, pp. 143-164. 
14. Gerrard, J.H., "The mechanics of the formation region of vortices behind bluff bodies", JFM, Vo1. 25, Part 2, 1966, pp. 401-413.

15. Griffin, 0.M., "The unsteady wake of an oscillating cylinder at low Reynolds numbers", ASME Journal of Applied Mechanics, paper no. 71-APM-33, Dec. 1971, pp. 729-738.

16. Griffin, 0.M., "Flow near self-excited and forced vibrating circular cylinders", ASME Journal of Engineering for Industry, Vo1. 94, May 1972, pp. 539-548.

17. Griffin, 0.M., "A universal Strouhal number for the '1ocking-on' of vortex shedding to the vibrations of bluff cylinders", JFM, Vo1. 85. Part 3, 1978, pp. 591-606.

18. Griffin, 0.M., "Vortex shedding from stationary and vibrating bluff bodies in a shear flow", NRL Memorandum Report 4287, Aug. 1980.

19. Griffin, O.M., "Universal similarity in the wakes of stationary and vibrating bluff structures", ASME Journal of Fluids Engineering, Vo1. 103, Mar. 1981, pp. 52-58. 
20. Griffin, O.M., Ramberg, S.E., Skop, R.A., Meggitt, D.J. and Sergev, S.S., "The strumming vibrations of marine cables: State of the art", Naval Civil Engineering Laboratory Technical Note, TN no. N-1608, May 1981.

21. Griffin, O.M. and Ramberg, S.E., "The vortex-street wakes of vibrating cylinders", JFM, Vol. 66, Part 3, 1974, pp. 553-576.

22. Griffin, O.M. and Ramberg, S.E., "On vortex strength and drag in bluff body wakes", JFM, Vol 69, Part 4, 1975, pp. 721-728.

23. Griffin, 0.M: and Votaw, C.W., "The vortex street in the wake of a vibrating cylinder", JFM Vo1. 51, Part 1, 1972, pp. 31-48.

24. Hoerner, S.F., Fluid Dynamic Drag, Hoerner Fluid Dynamics, New Jersey, 1965.

25. Koopmann, G.H., "The vortex wakes of vibrating cylinders at low Reynolds numbers", JFM, Vo1. 28, Part 3, 1967, pp. 501-512.

26. Mair, W.A. and Stansby, P.K., "Vortex wakes of bluff cylinders in a shear flow", SIAM Journal of Applied Mathematics, Vo1. 28, 1975, pp. 519-540. 
27. Maul1, D.J. and Young, R.A., "Vortex shedding from bluff bodies in a shear flow," JFM, Vo1. 60, 1973, Part 2, pp. 401-409.

28. Newland, D.E., An Introduction to Random Vibrations and Spectral Analysis, Longman Group Limited, London, 1975.

29. Pao, H.P. and Kao, T.W., "Vortex structure in the wake of a sphere", Physics of Fluid, Vo1. 20. No. 2, 1977, pp. 187-191.

30. Parkison, G.V., "Aspects of the aeroelastic behavior of bluff cylinders", Published by Engineering Institute of Canada, Montrea1, Canada, 1961.

31. Peltzer, R.D. and Rooney, D.M., "Wake characteristics of high aspect ratio cylinders in subcritical spanwise sheared flows", ASME Publication 81-WA/FE-10, Nov. 1981.

32. Peltzer, R.D., "The effect of upstream shear and surface roughness on the vortex shedding patterns and pressure distributions aroung a circular cylinder in transitional Reynolds number flows", M.S. Thesis, VPI\&SU, Apr. 1980, 176 pp.

33. Physitech Inc., Technical Instruction Manual for the Model 440 Auto Collimator, 1968, 43 pp. 
34. Ramberg, S.E., "The influence of yaw angle upon the vortex wakes of stationary and vibrating cylinders", NRL Memorandum Report 3822, Aug. 1978, 114 pp.

35. Ramberg, S.E. and Griffin, O.M., "Vortex formation in the wake of a vibrating, flexible cable", ASME Journal of Fluids Engineering, Dec. 1974, pp. 317-322.

36. Ramberg, S.E. and Griffin, 0.M., "The effects of vortex coherence, spacing and circulation on the flow-induced forces on vibrating cables and bluff structures", NRL Report 7945, Jan. 1976, 32 pp.

37. Ramberg, S.E. and Griffin, O.M., "Velocity correlation and vortex spacing in the wake of a vibrating cable", ASME Jouranl of Fluids Engineering, Vo1. 98, Mar. 1976, pp. 10-18.

38. Rooney, D.M. and Peltzer, R.D., "Pressure and vortex shedding patterns around a low aspect ratio cylinder in a sheared flow at transitional Reynolds numbers," ASME Journal of Fluids Engineering, Mar. 1981, pp. 88-96.

39. Rooney, D.M. and Peltzer, R.D., "The effects of roughness and shear on vortex shedding cell lengths behind a circular cylinder", ASME Journal of Fluids Engineering, Vol. 104, No. 1, Mar. 1982, pp. $72-80$ 
40. Roshko, A., "On the wake and drag of bluff bodies", Journal of Aeronautical Sciences, Vo1. 22, 1955, pp. 124-132.

41. Sarpkaya, T., "Vortex-induced oscillations; A selective revi ew", ASME Journal of Applied Mechanics, Vol. 46, Jun. 1979, pp. 241258.

42. Sarpkaya, T. and Shoaff, R.L., "A discrete-vortex analysis of flow about stationary and transversely oscillating circular cylinders", Nava 1 Postgraduate School Report NPS-69SL7 9011, Jan. 1979, 166 pp.

43. Schaefer, J.W. and Eskinazi, S., "An analysis of the vortex street generated in a viscous fluid", JFM, Vol. 6, 1959, pp. 241-260.

44. Stansby, P.K., "The locking-on of vortex shedding due to the cross stream vibration of circular cylinders in uniform and shear flows", JFM, Vo1. 74, Part 4, 1976, pp. 641-665.

45. Sullerey, R.K., Gupta, A.K. and Moorthy, C.S., "Similarity in the turbulent near wake of bluff bodies", AIAA Journal, Nov. 1975, pp. 1425-1429. 
46. Vandiver, J. K. and Griffin, O. M., "Measurements of the VortexExcited Strumming Vibrations of Marine Cables", Ocean Structural Dynamics Symposium '82 Preprints, Oregon State University, to appear, September 1982.

47. Walker, S., "Helical strake kit to reduce flow-induced vibration of single pile aids to navigation," Engineers Digest, 1980, pp. $22-25$.

48. Woo, H.G.C., Cermak, J.E. and Peterka, J.A., "Experiments on vortex shedding from stationary and oscillating cables in a linear shear flow," Final report on contract N68305-78-C-0055 for the Naval Civil Engineering Laboratory, Colorado State University, Ju1. 1981, $223 \mathrm{pp}$.

49. Wootton, L.R., Warner, M.H., Sainsbury, R.N. and Cooper, D.H., "Oscillation of piles in marine structures," CIRIA Technical Note 41, Aug. 1972.

50. Zdravkovich, M.M., "Modification of vortex shedding in the synchronization range," ASME paper 81-WA/FE-25, Nov 1981,8 pp. 
Appendix A

Figures 


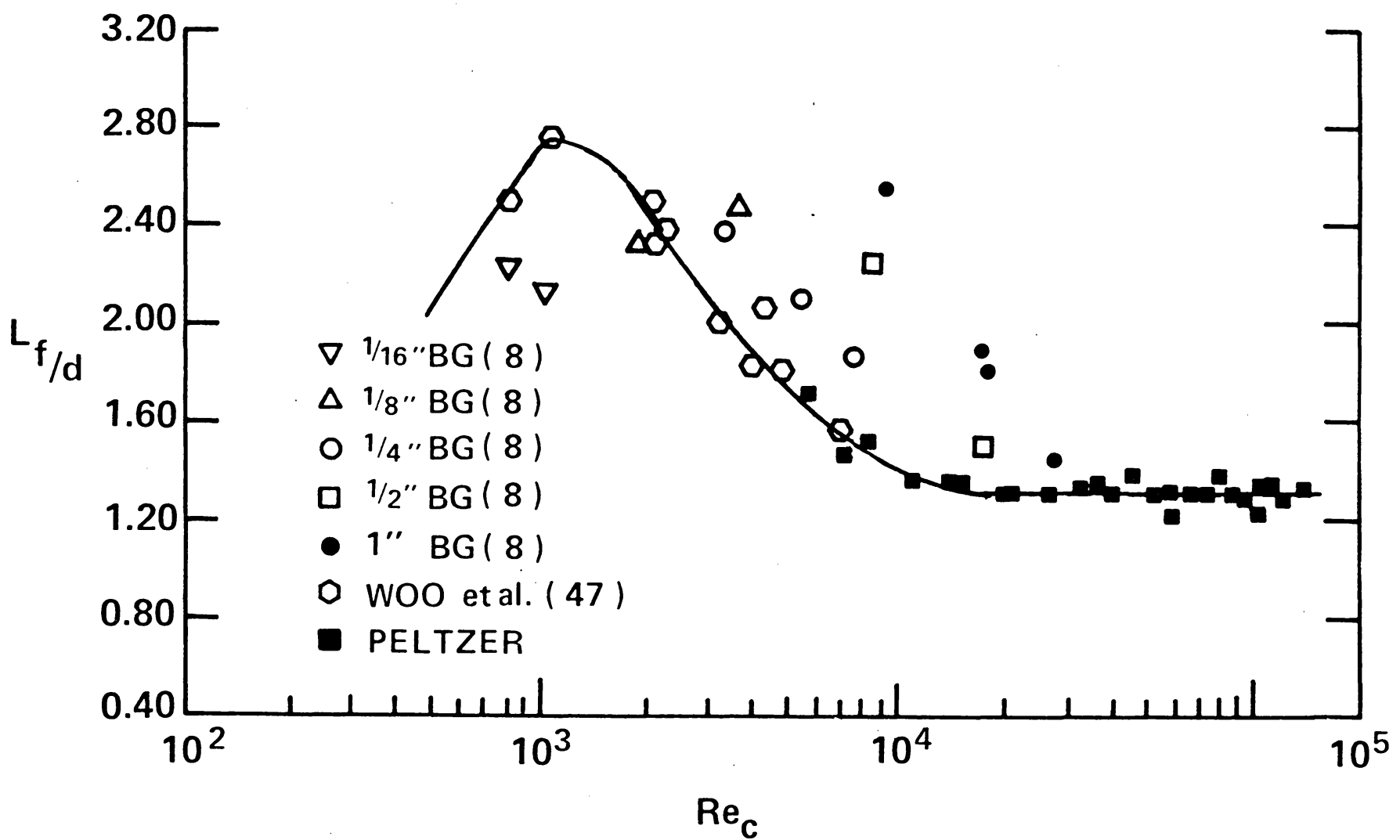

Figure 1. Vortex Formation Lengths for Circular Cylinders in Uniform and Linear Shear Flows. 


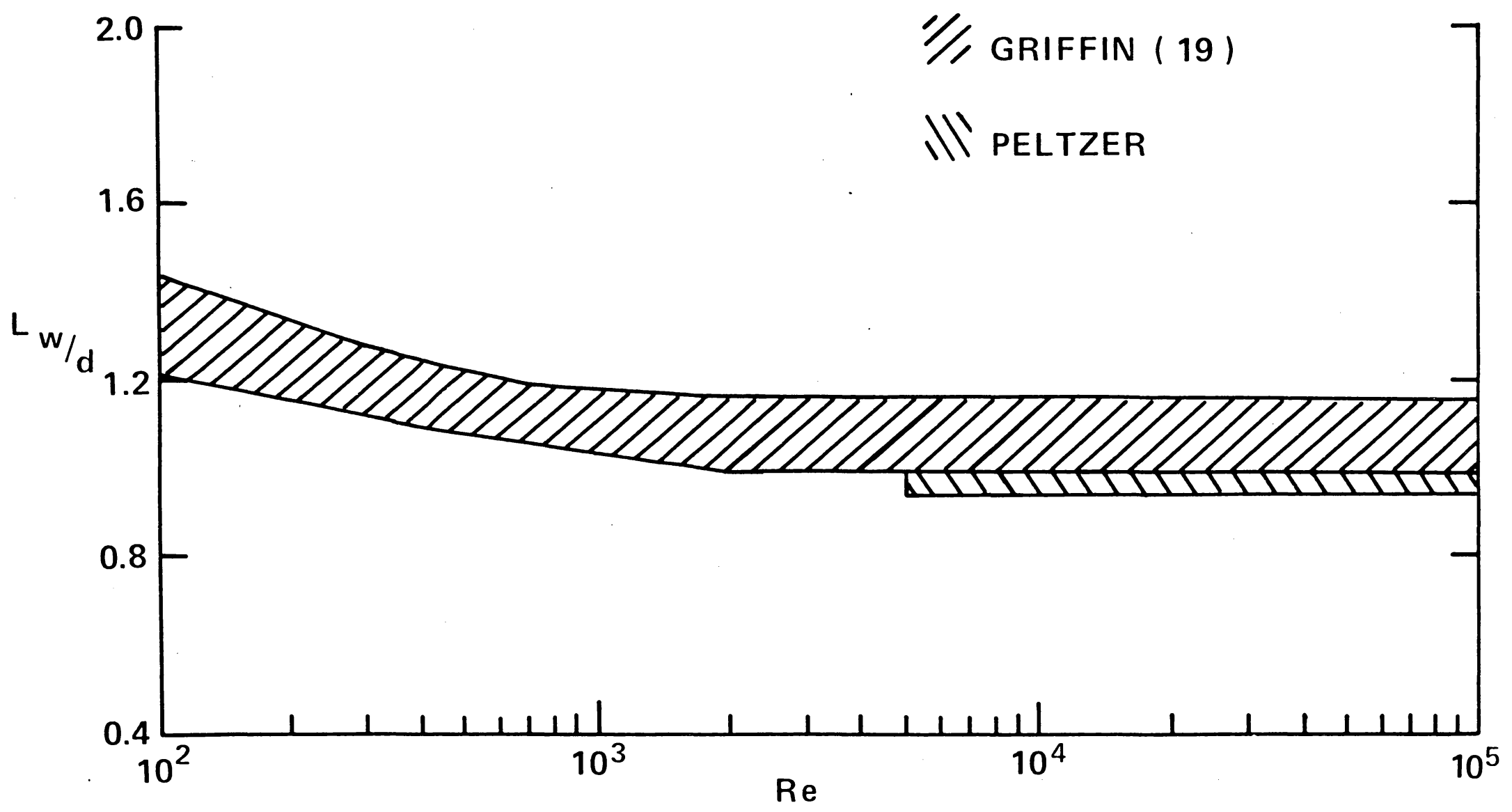

Figure 2. Wake Widths for Circular Cylinders in Uniform Flow. 


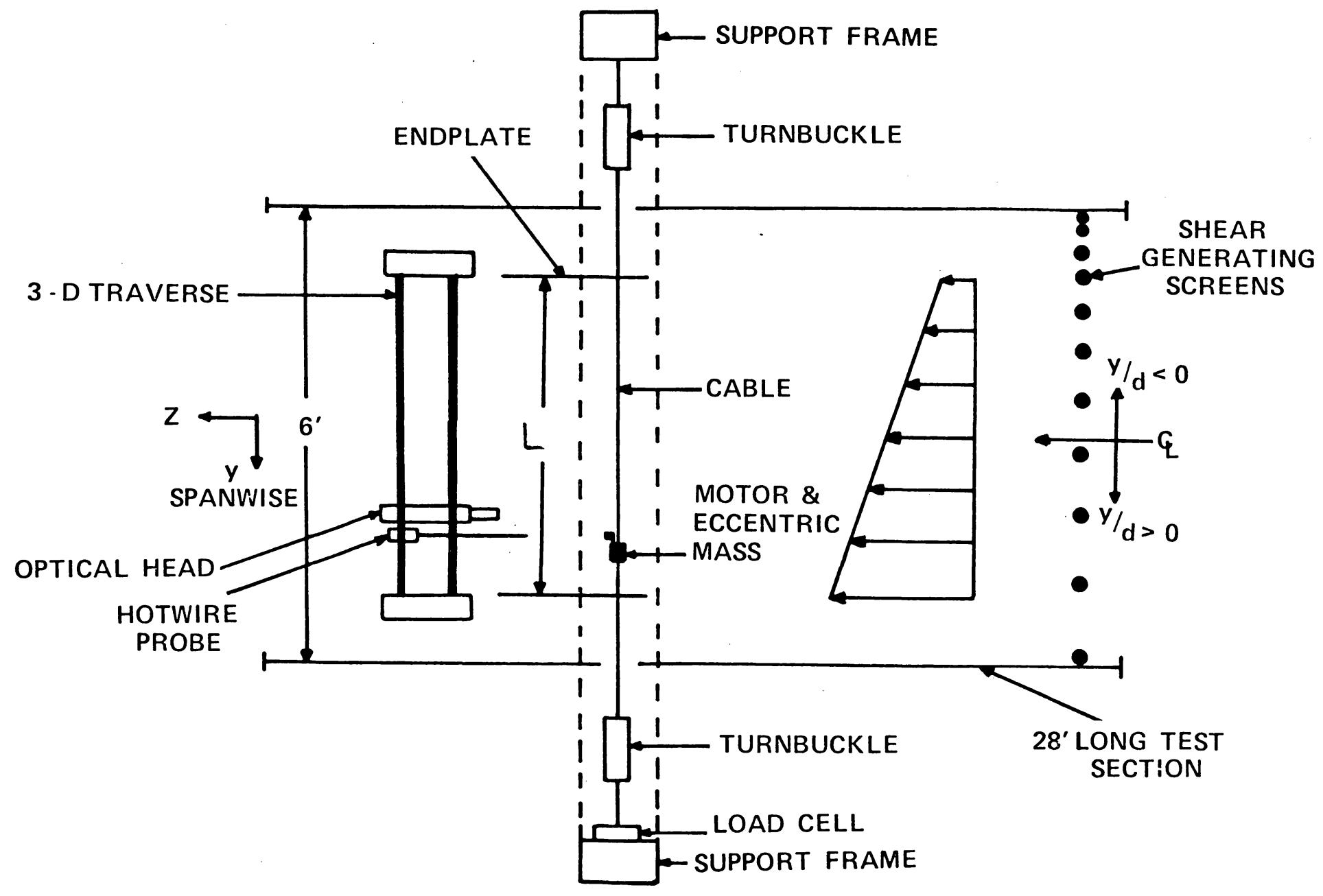

Figure 3. Wind Tunnel Test Section and Cable Model (Top View). 


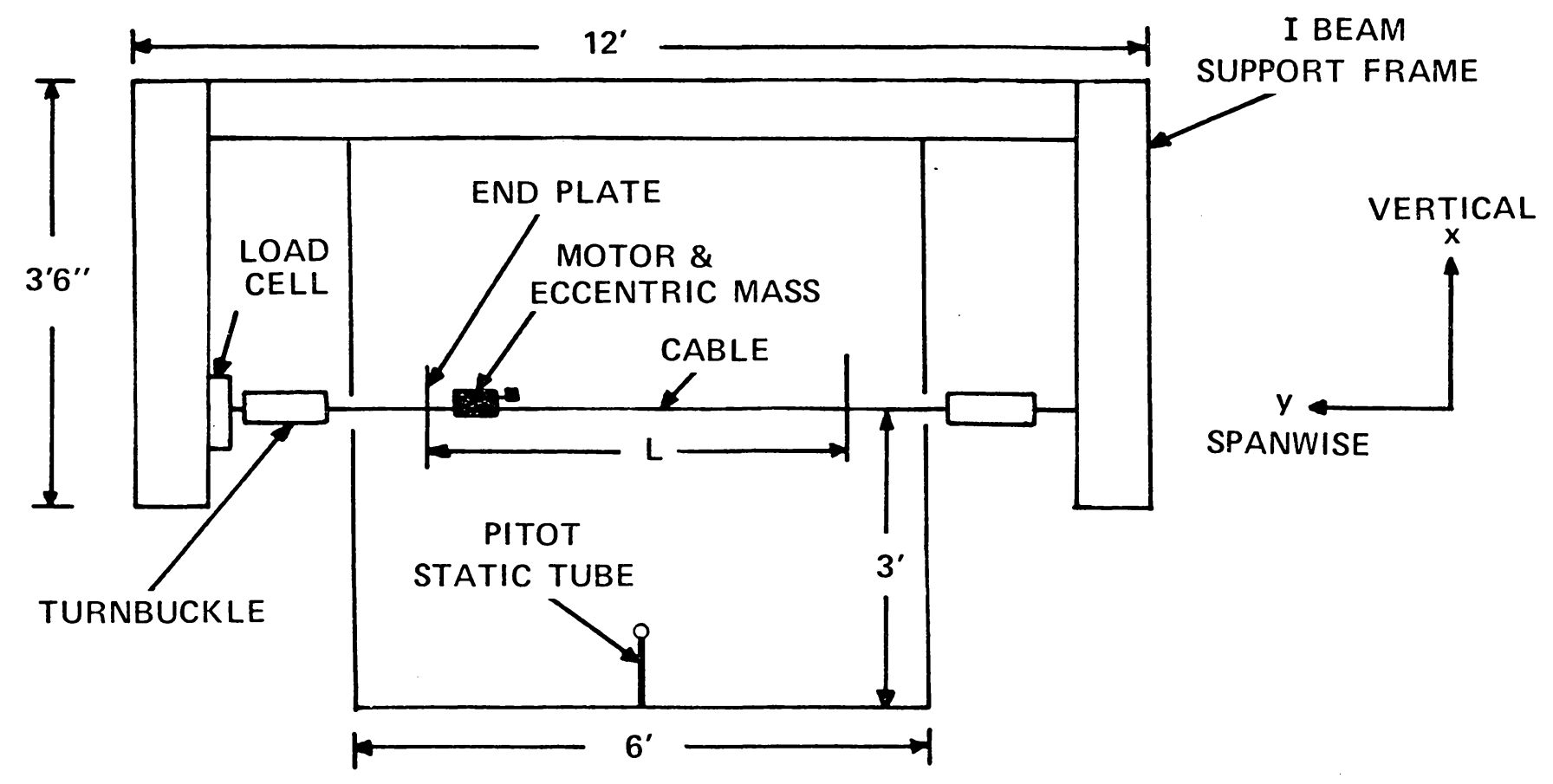

Figure 4. Wind Tunnel Test Section and Cable Model (Downstream View). 


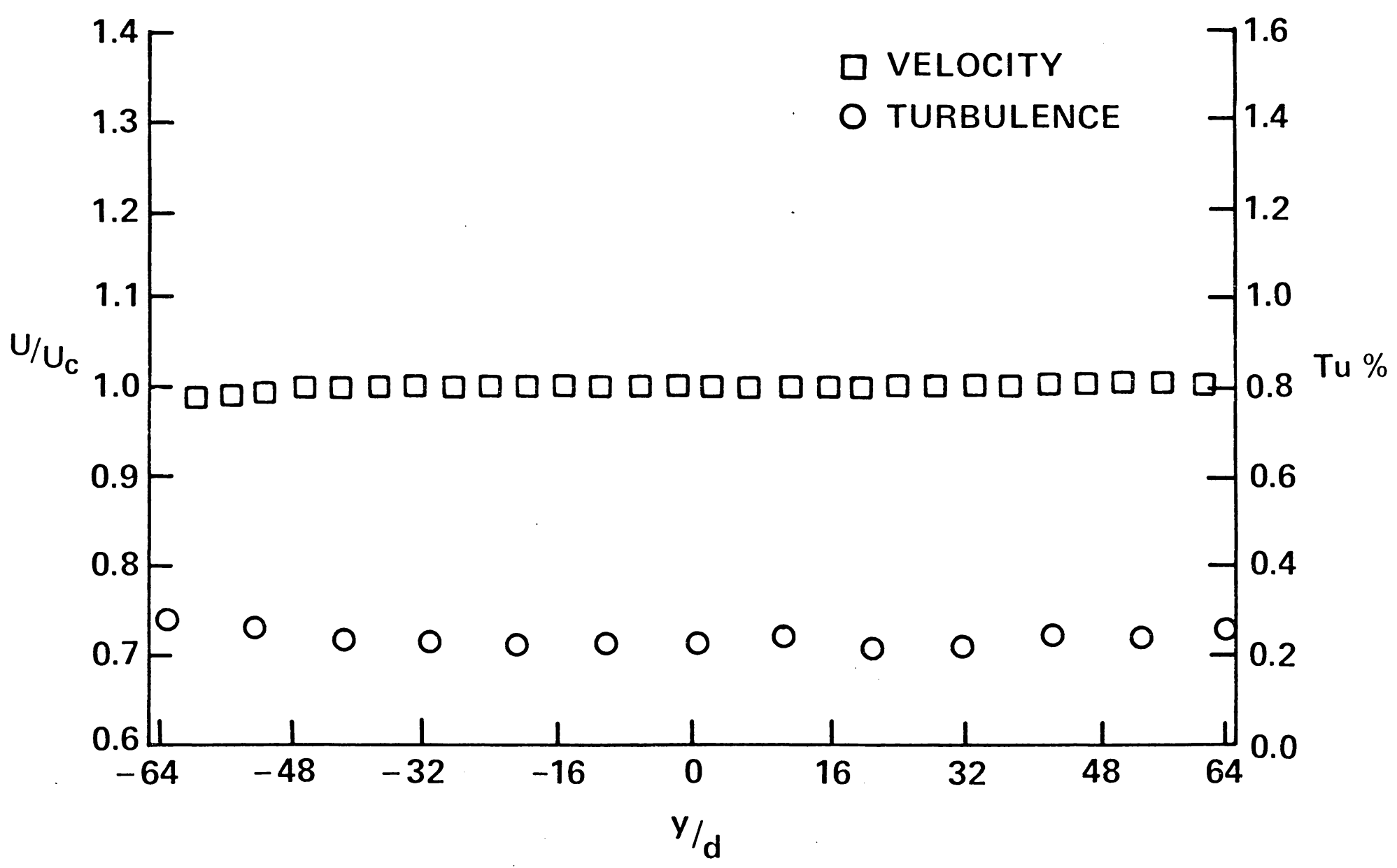

Figure 5. Spanwise Velocity and Turbulence Profiles $\beta=0.0$. 


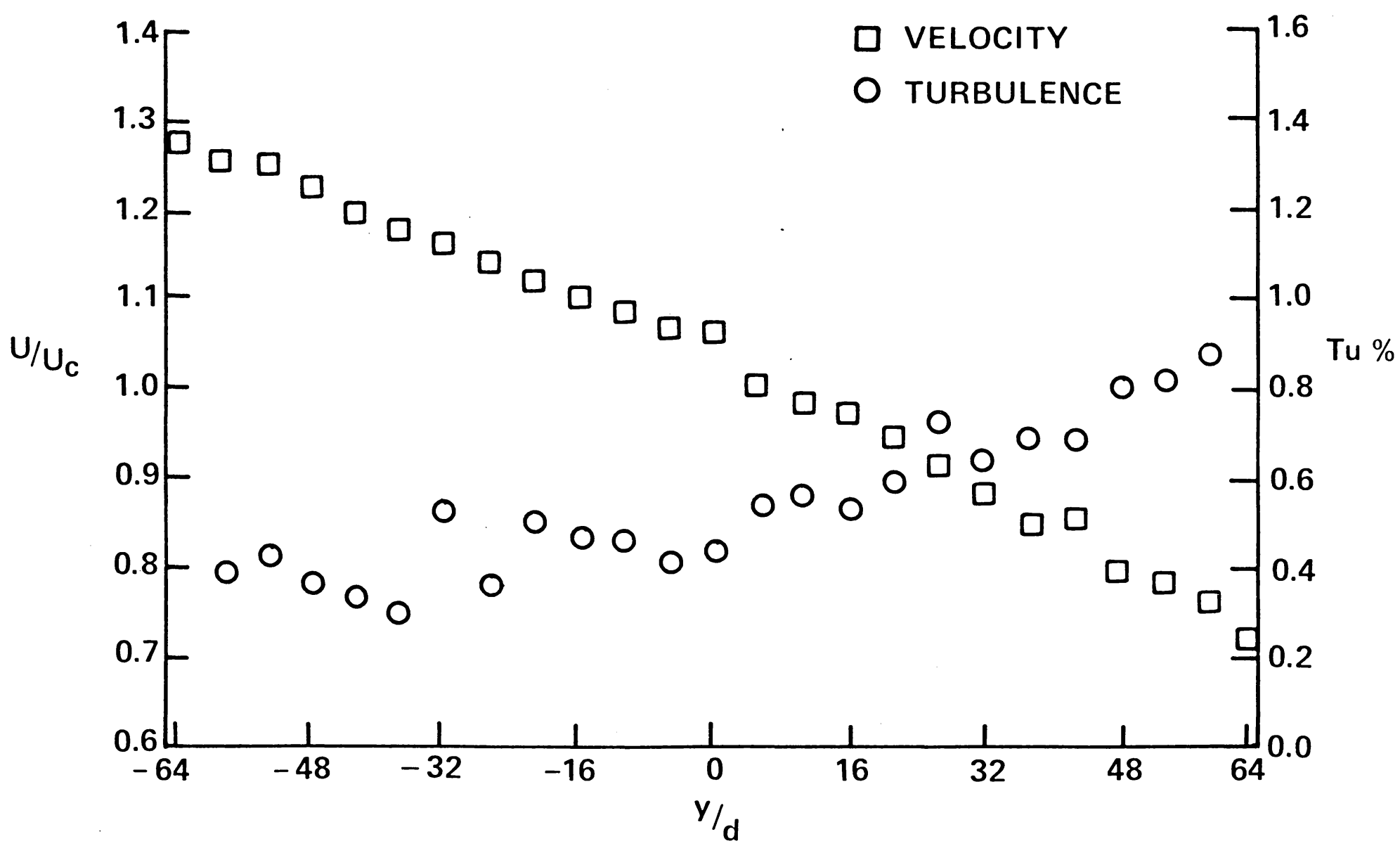

Figure 6. Spanwise Velocity and Turbulence Profiles $\beta=0.0053$. 


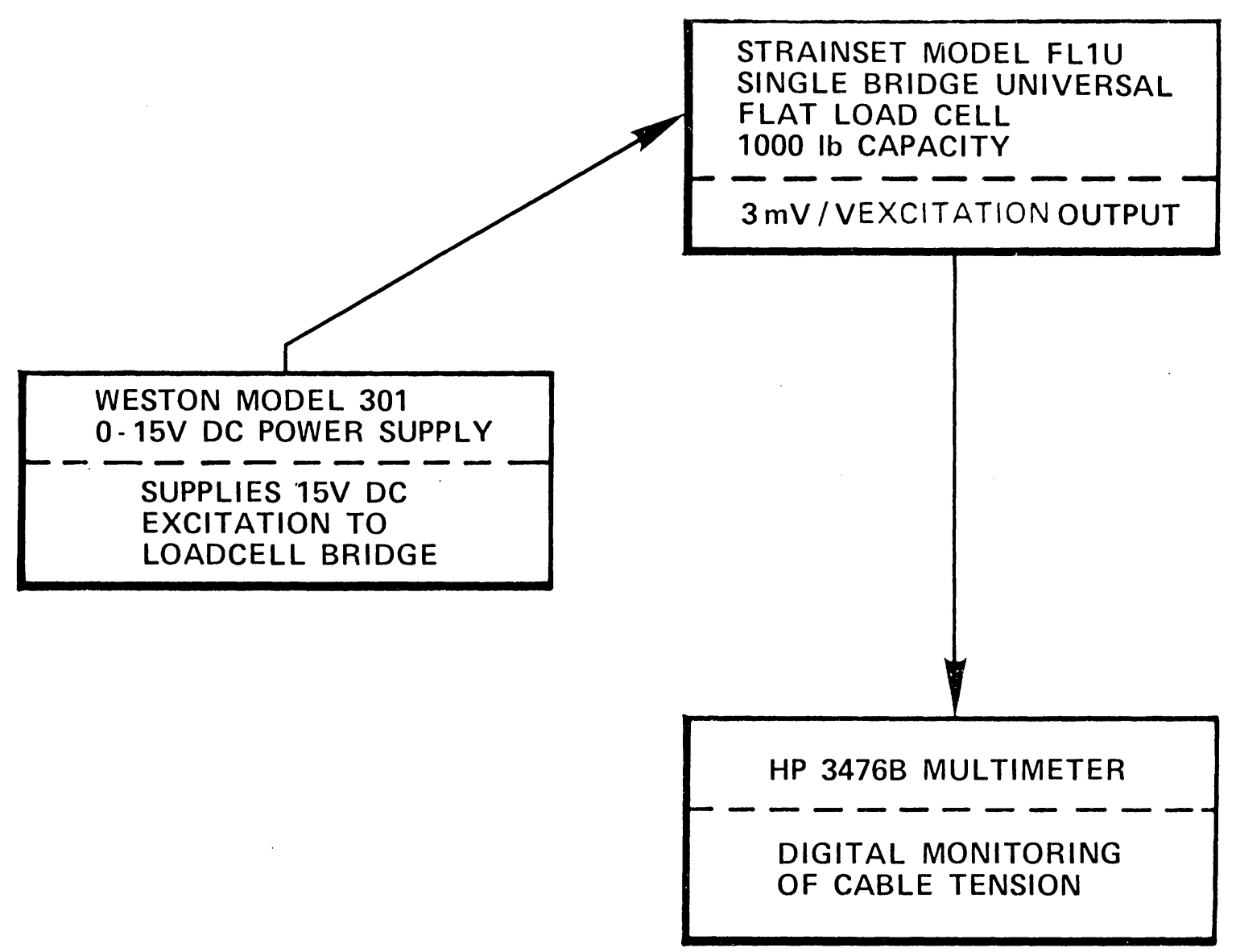

Figure 7. Cable Tension Masurement. 


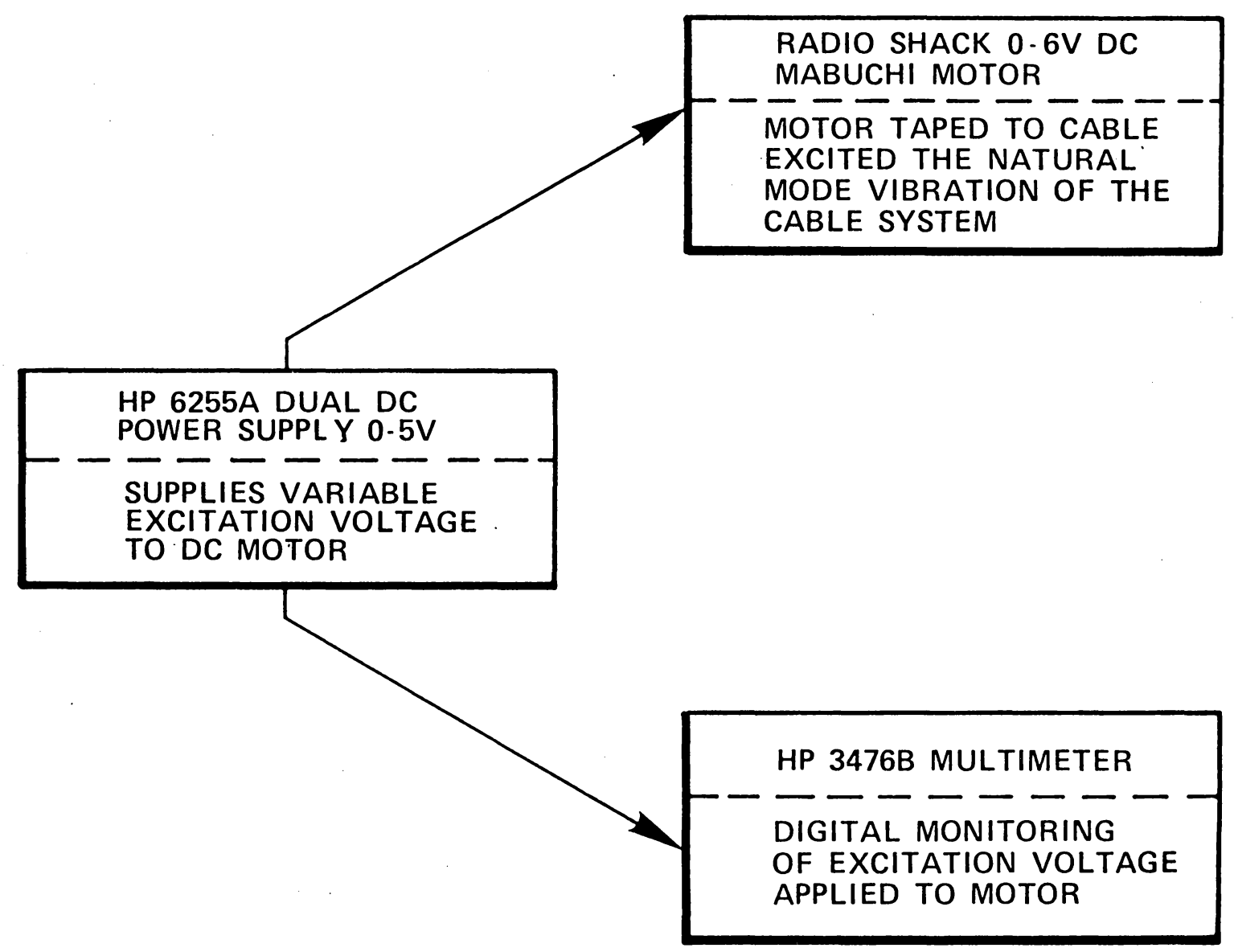

Figure 8. Forced Cable Vibration. 


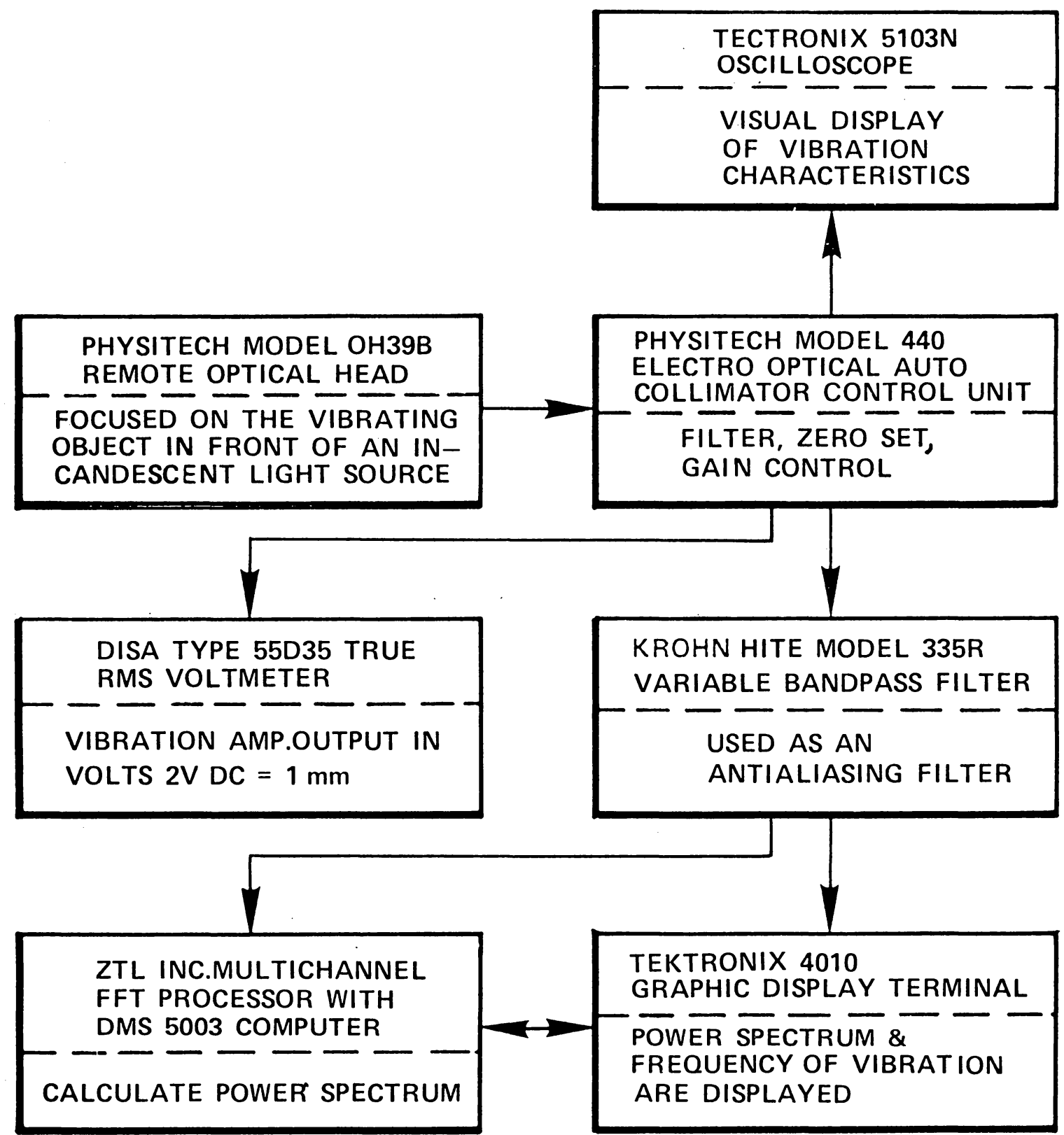

Figure 9. Cable Mode Shape, Vibration Amplitude and Frequency Measurement. 


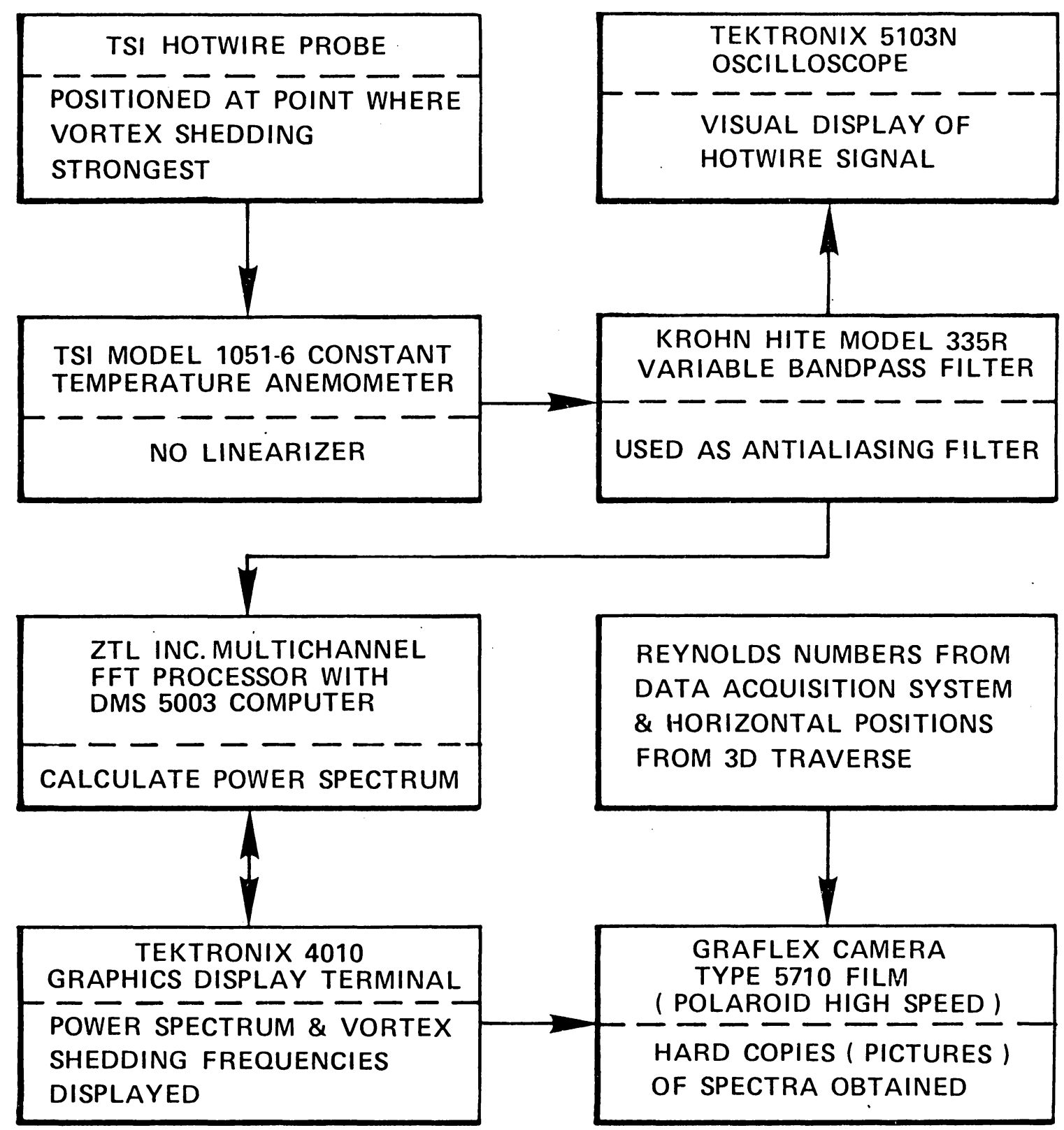

Figure 10. Vortex Shedding Frequencies Me asurement. 


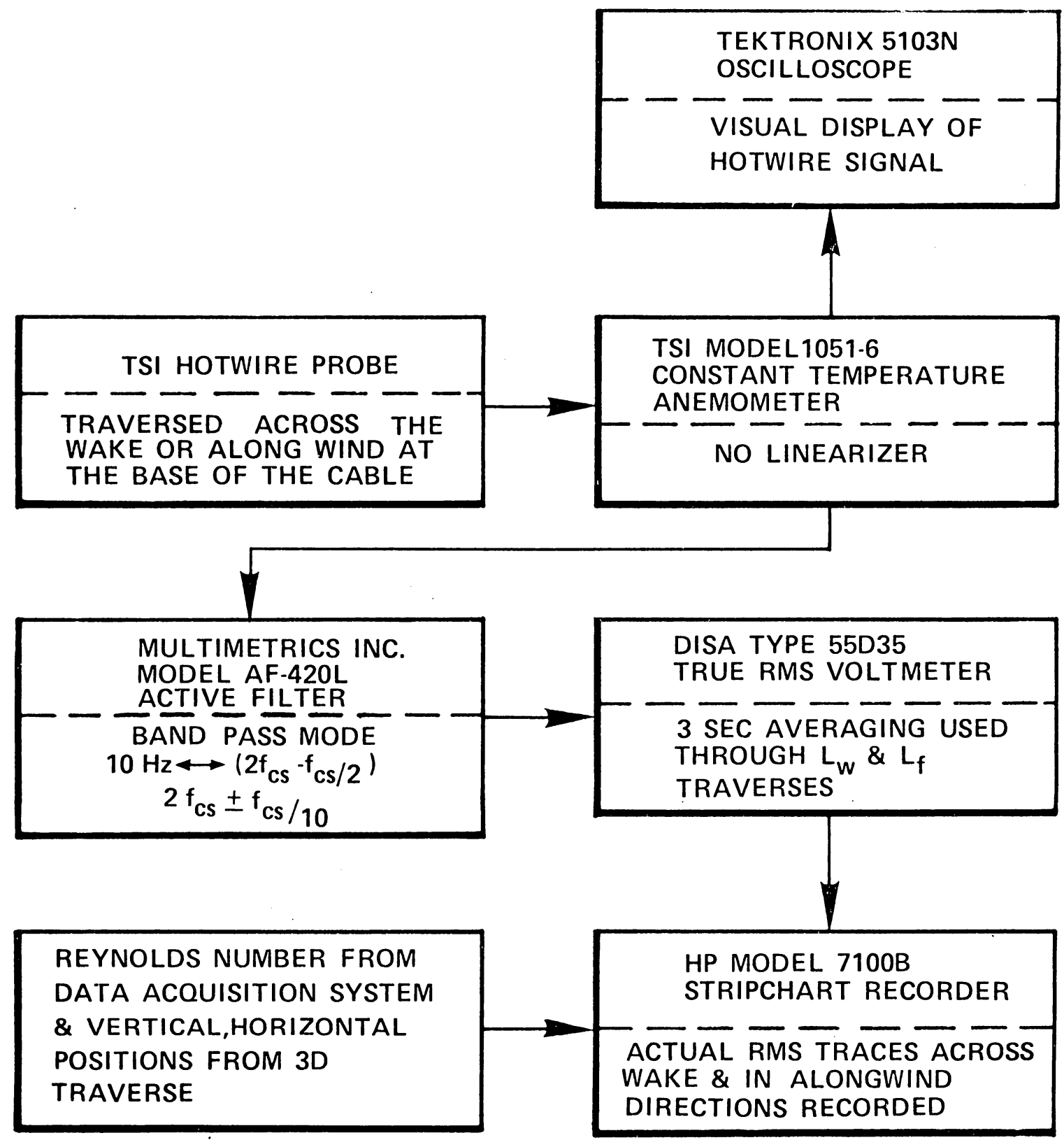

Figure 11. Cable Wake Width and Vortex Formation Length Instrume ntation. 


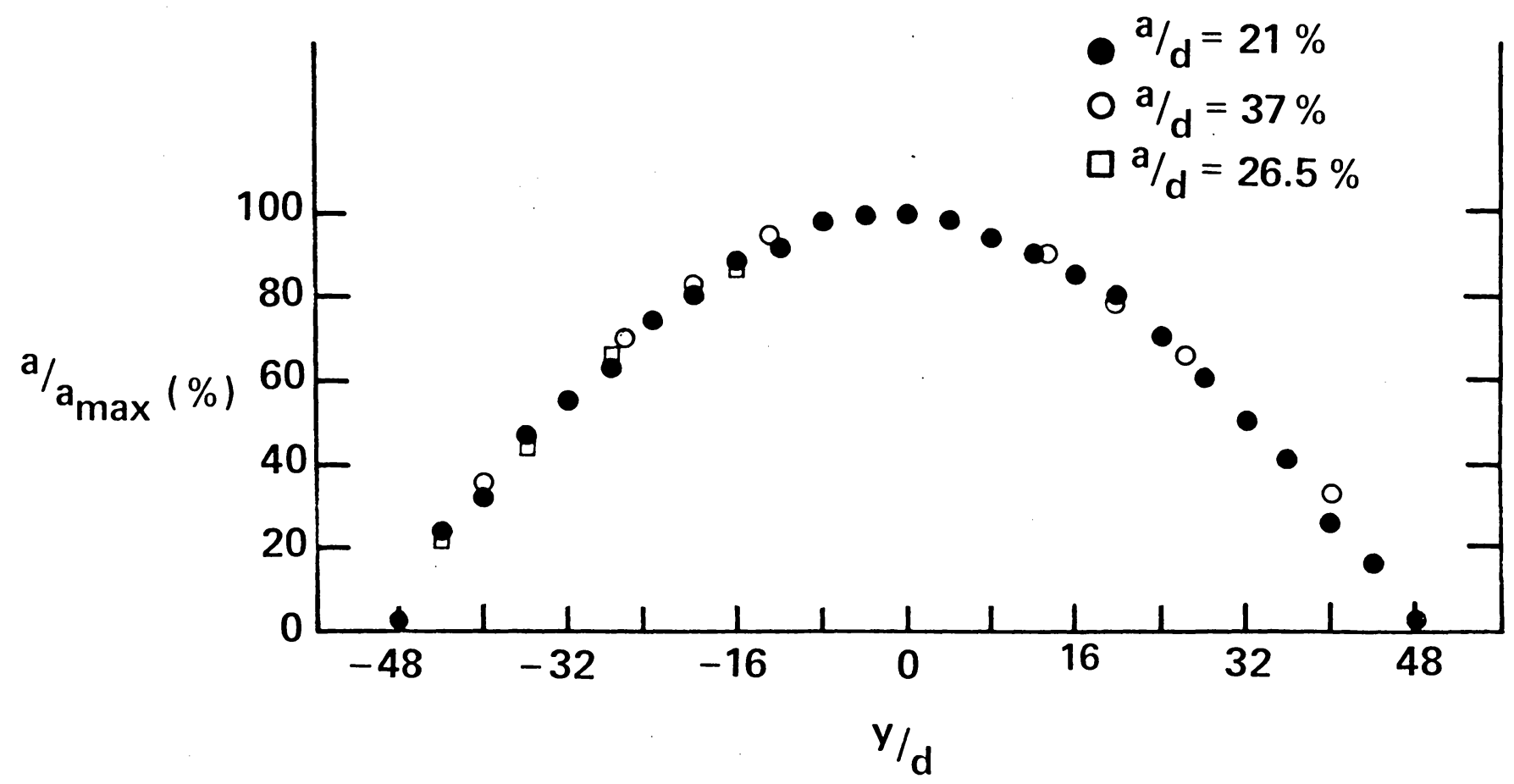

$\underset{\infty}{\rightleftarrows}$

Figure 12. Cable Mode Shape (First Mode). 


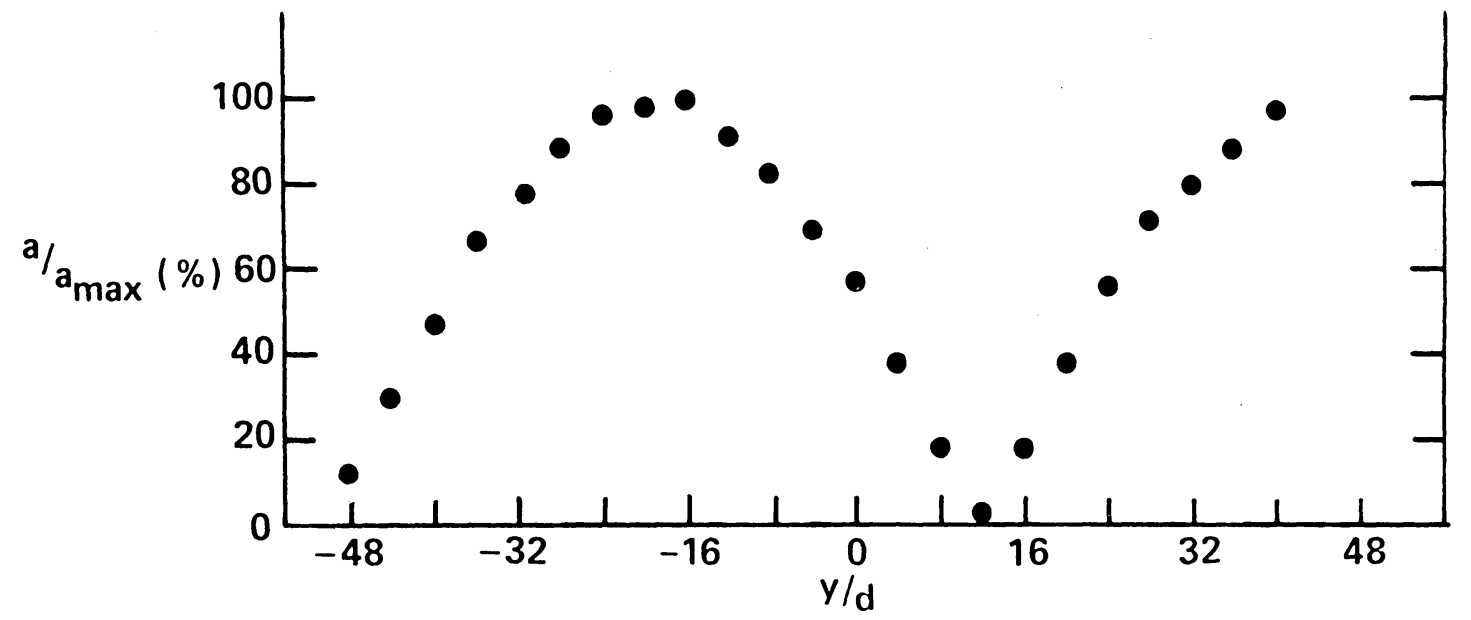

(a) SECOND PHASE TESTS

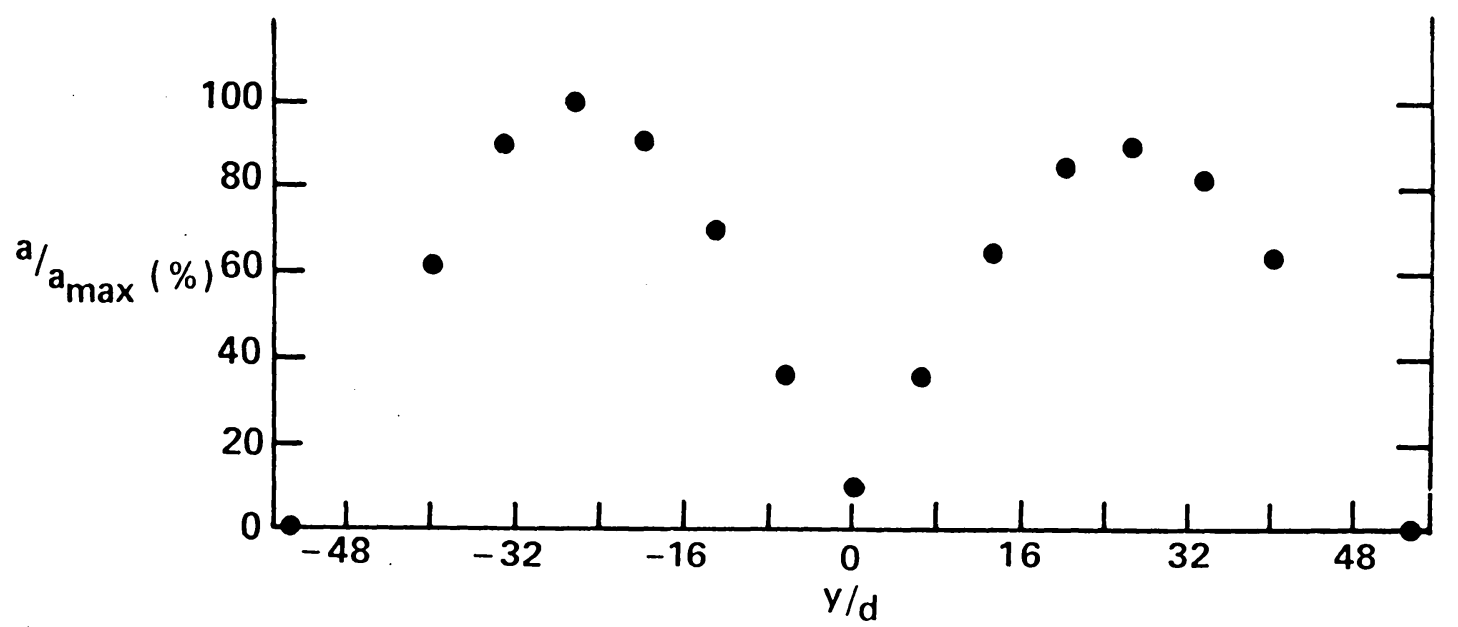

(b) FIRST PHASE TESTS

Figure 13. Cable Mode Shape (Second Mode). 


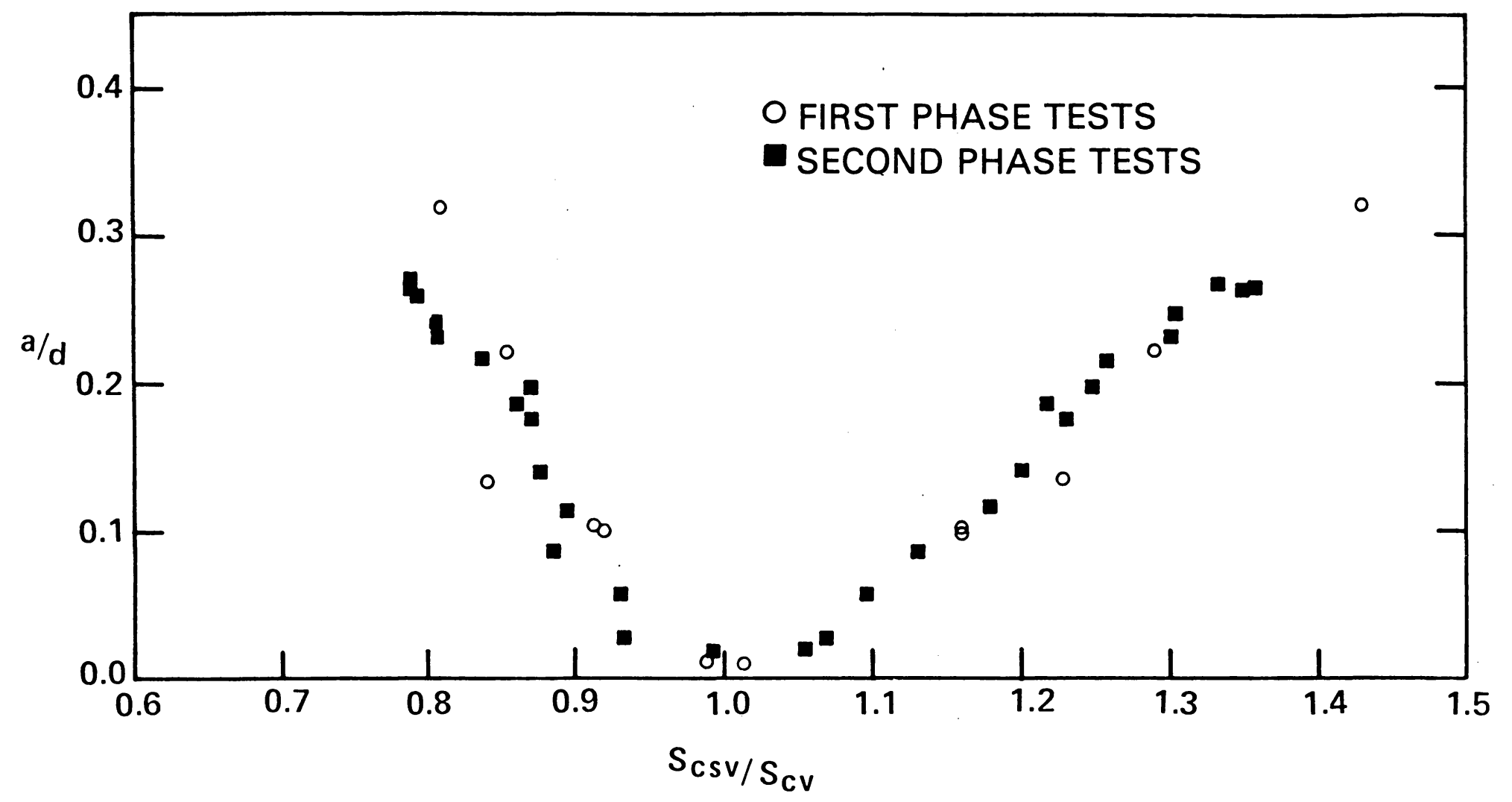

Figure 14. Cable Lock-on Regions as a Function of Vibration Amplitude. 


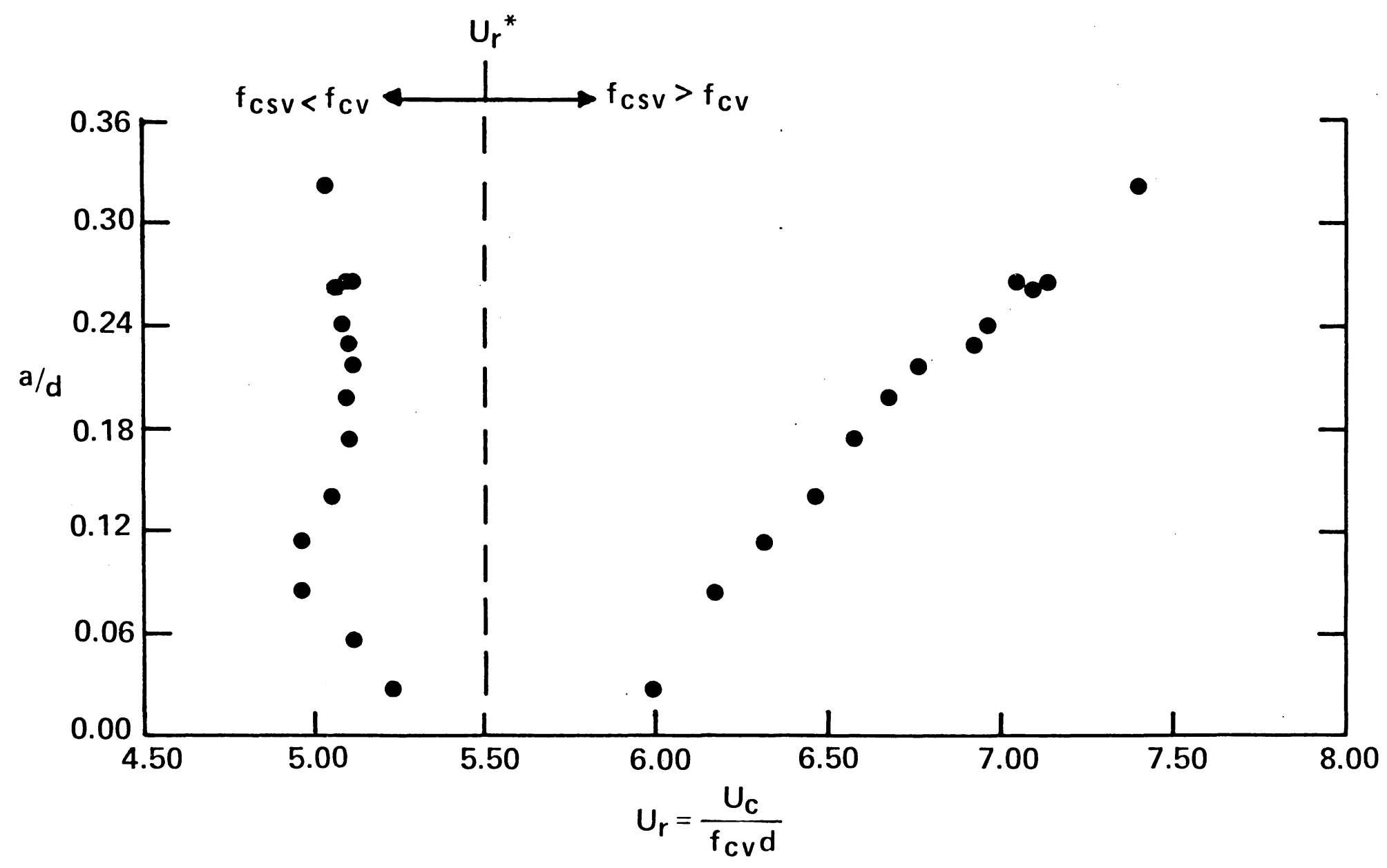

Figure 15. Reduced Velocity at the Boundaries of the Lock-on Region for the Vibrating Cable as Function of the Vibration Amplitude. 


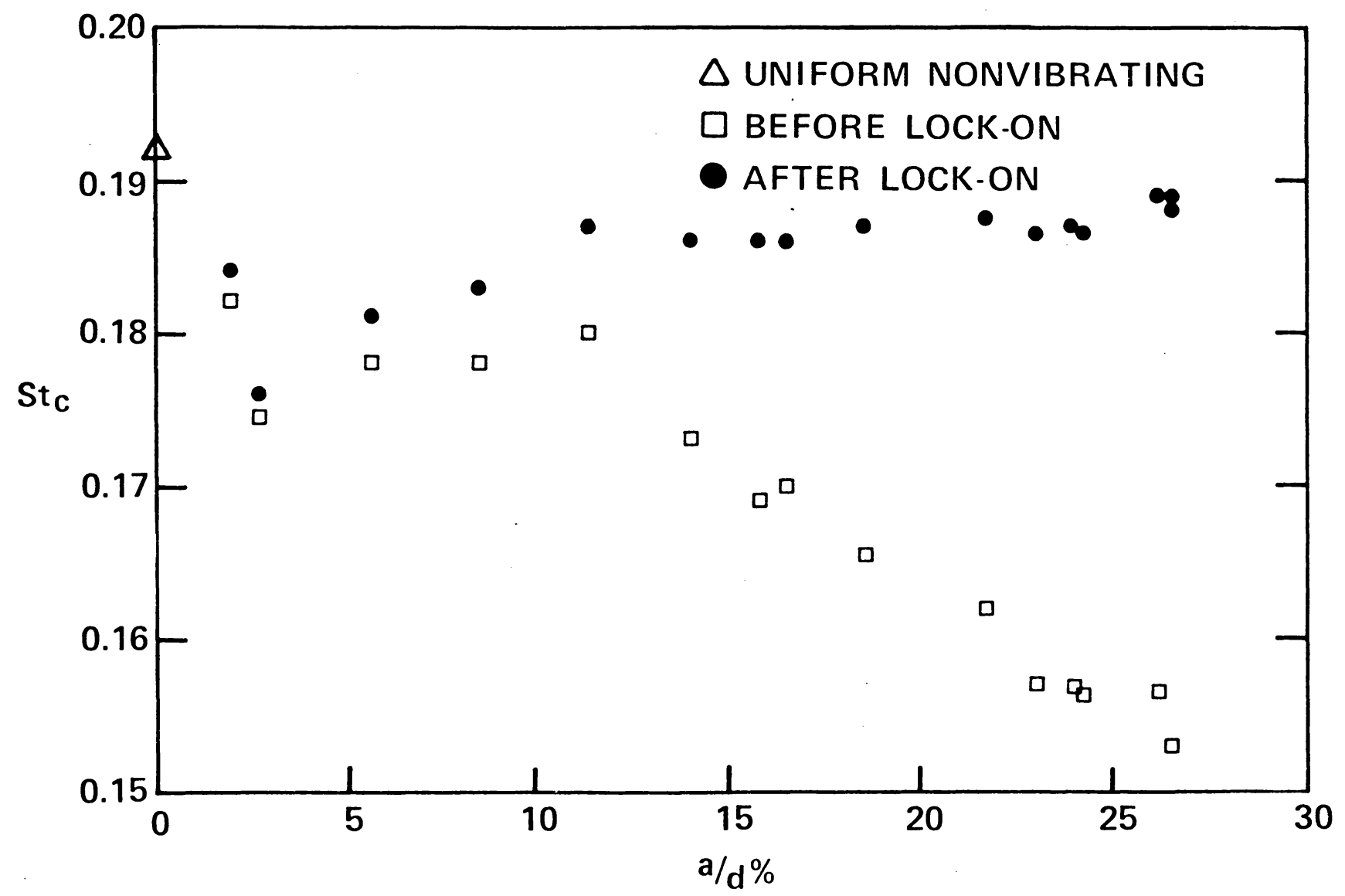

Figure 16. Variation of the Strouhal Number as a Function of Vibration Amplitude at the Boundaries of the Lock-on Region. 


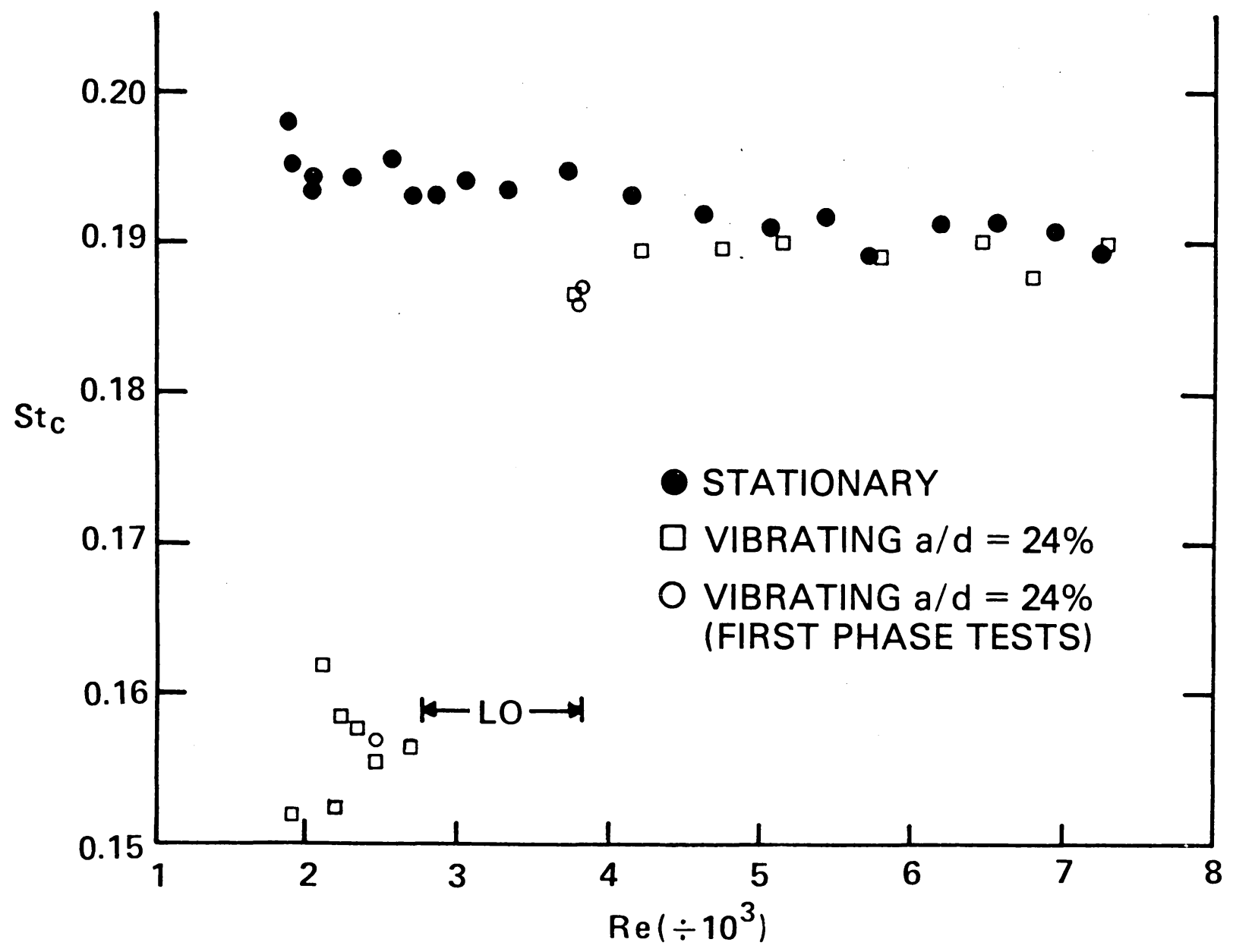

Figure 17. Variation of the Centerline Strouhal Number as a Function of Reynolds Number of a Stationary and Vibrating Cable. 

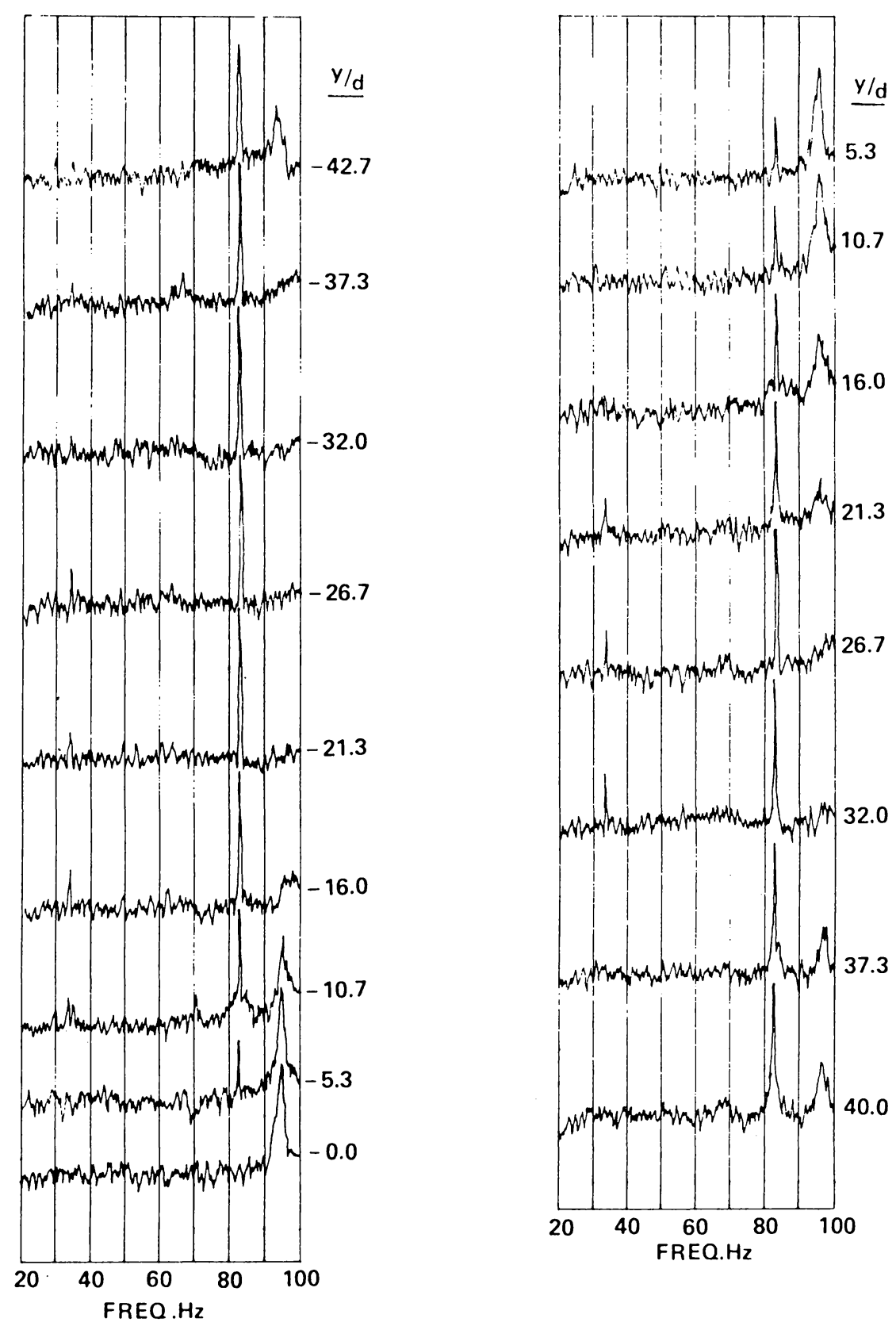

Figure 18. Spanwise Behavior of the Vortex Shedding Frequency during Lock-on, $\operatorname{Re}_{\mathrm{c}}=3.83 \times 10^{3}$, Second Mode Shape, $a / d=11.7 \%, \beta=0.0$. 


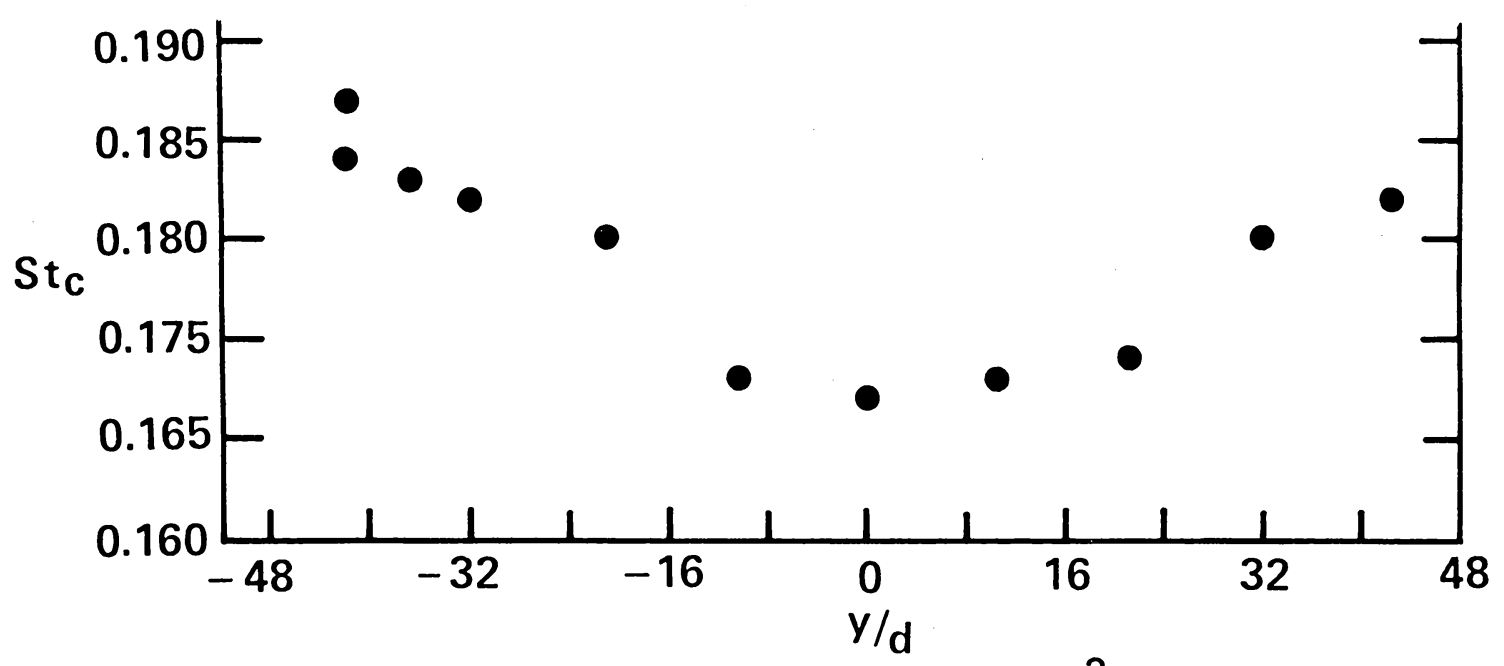

(a) FIRST PHASE TEST, $\operatorname{Re}_{\mathrm{c}}=1.9 \times 10^{3}, \mathrm{a} / \mathrm{d}=32.1 \%$ FIRST MODE

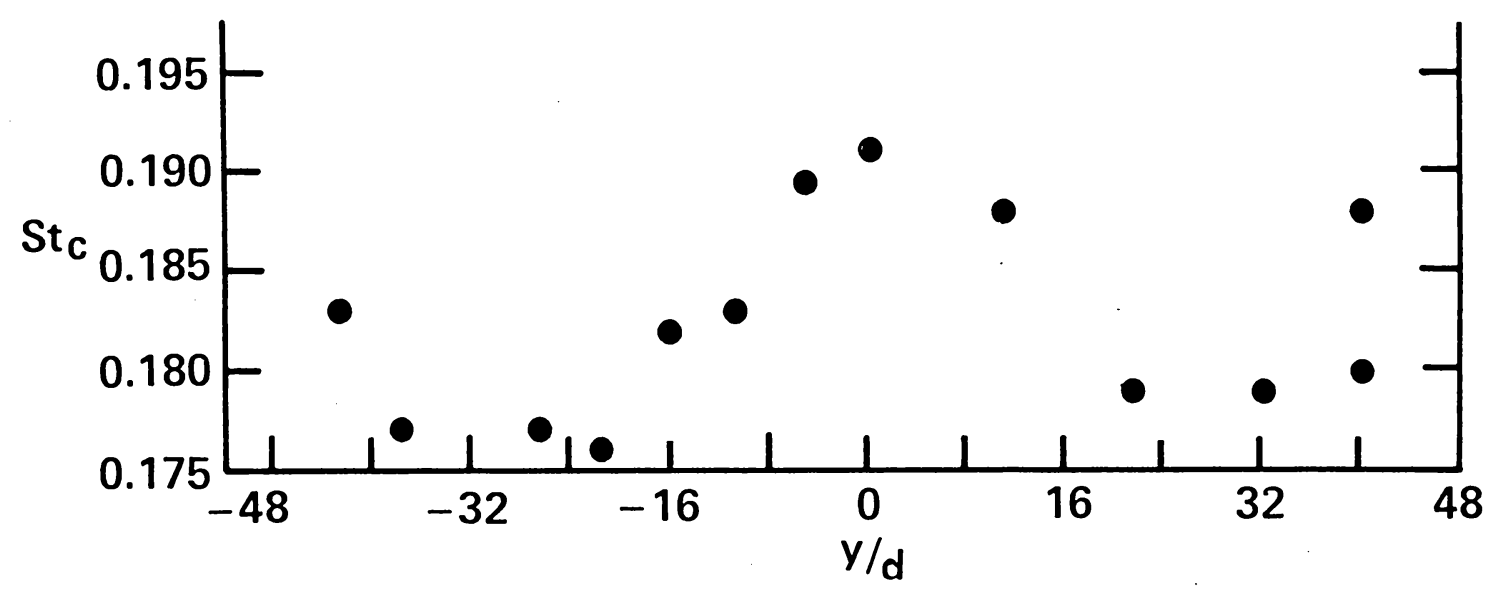

(b) FIRST PHASE TEST, $\operatorname{Re}_{\mathrm{c}}=2.67 \times 10^{3}, \mathrm{a} / \mathrm{d}=10.2 \%$ SECOND MODE

Figure 19. Spanwise Variation of the Vortex Shedding Frequency below the Lock-on Range, $\beta=0.0$. 


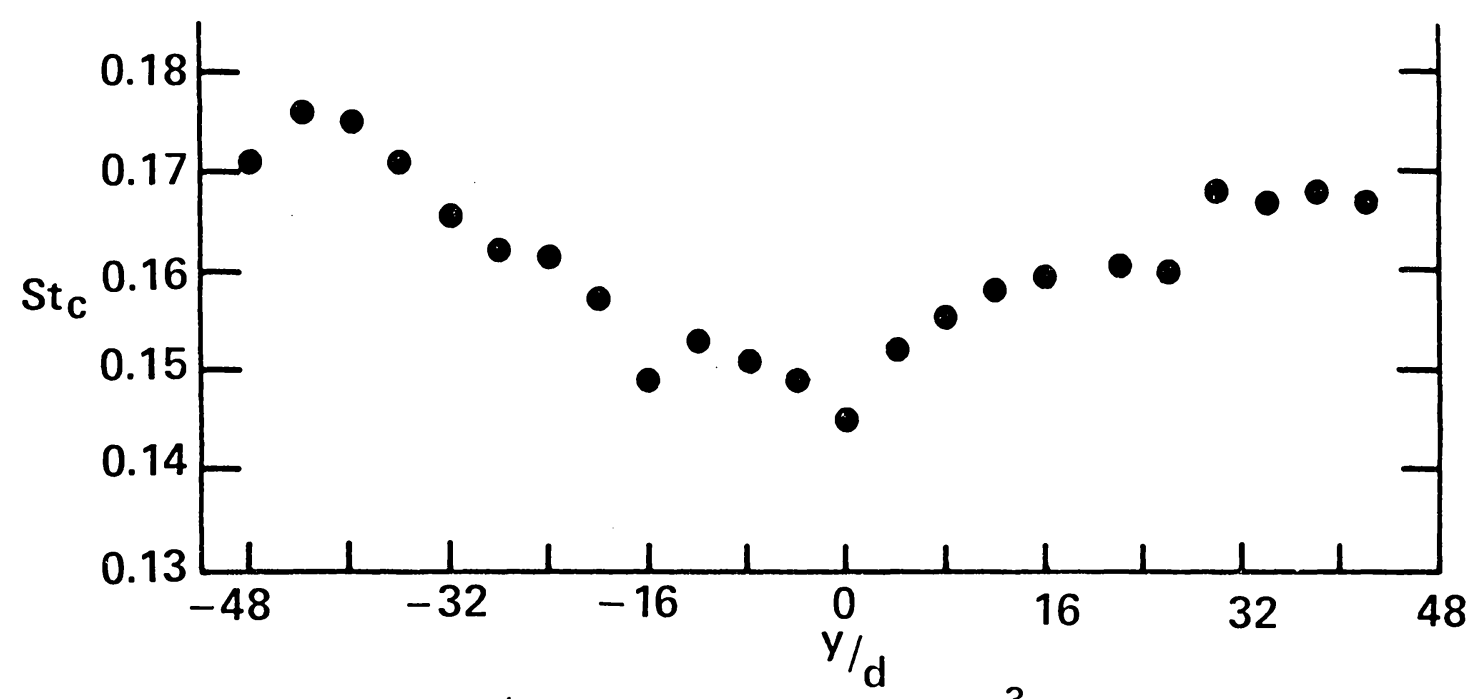

(a) $a / d=25.4 \%, \operatorname{Re}_{c}=2.10 \times 10^{3}$

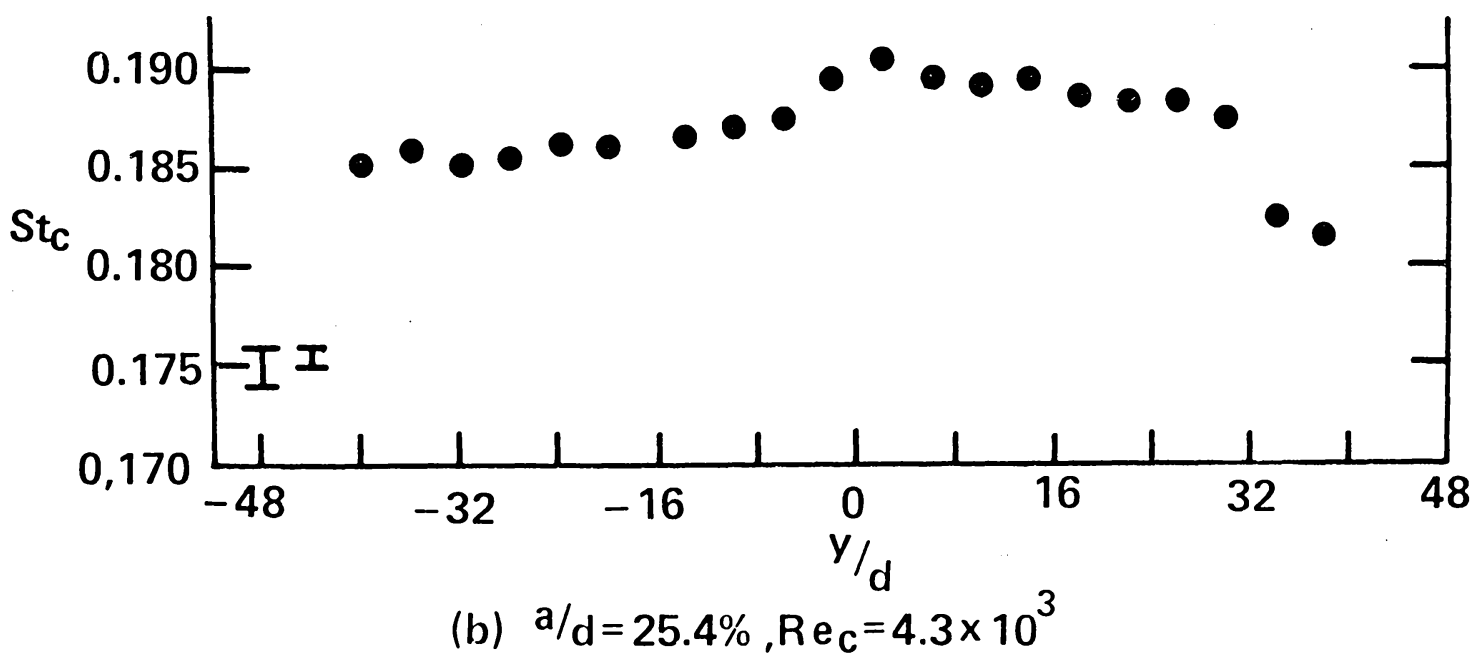

Figure 20. Spanwise Variation of the Vortex Shedding Frequency away from the Lock-on Region, First Mode, $\beta=0.0$. 


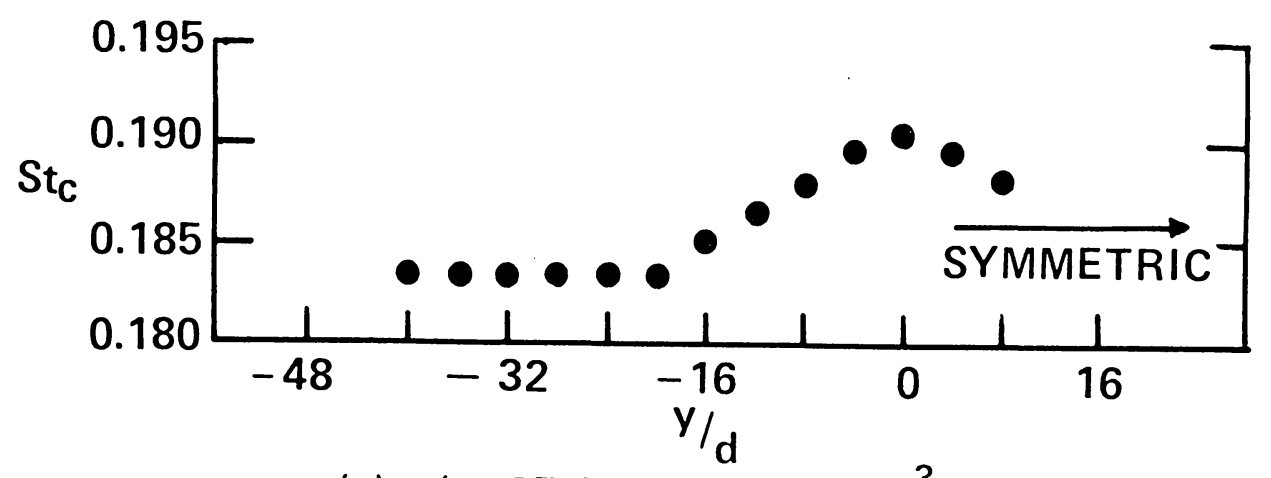

(a) $a / d=25.4 \%, \operatorname{Re}_{c}=5.75 \times 10^{3}$

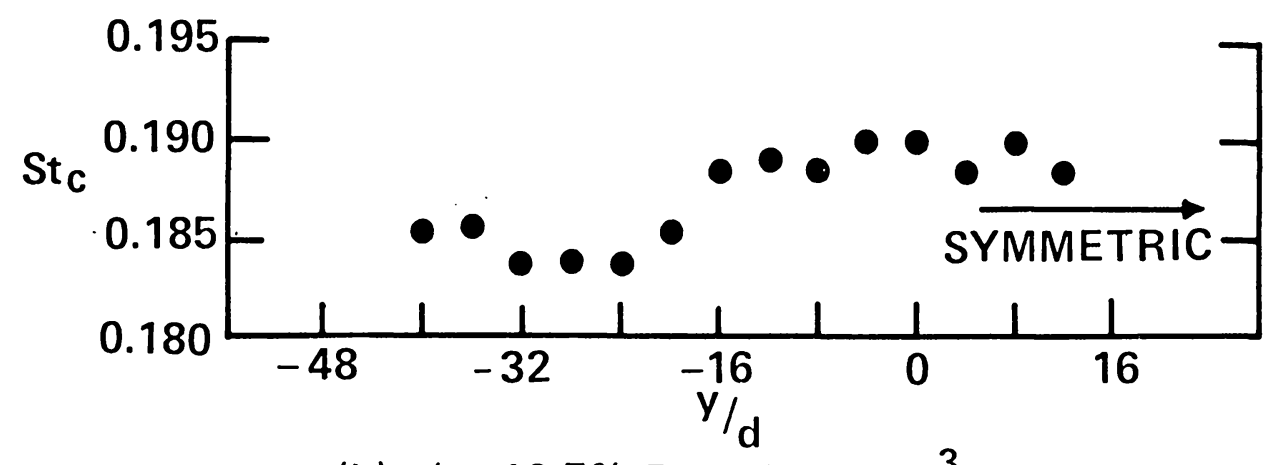

(b) $a / d=10.5 \%, R_{c}=5.78 \times 10^{3}$

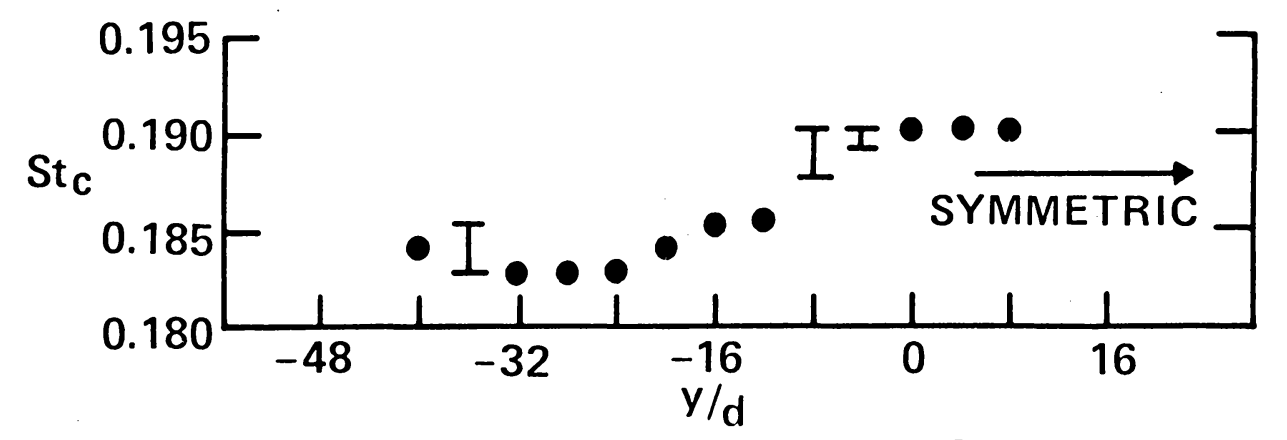

(c) $a / d=25.4 \%, \operatorname{Rec}=6.9 \times 10^{3}$

Figure 21. Sparwise Variation of the Vortex Shedding Frequency away from the Lock-on Range, First Mode, $\beta=0.0$. 


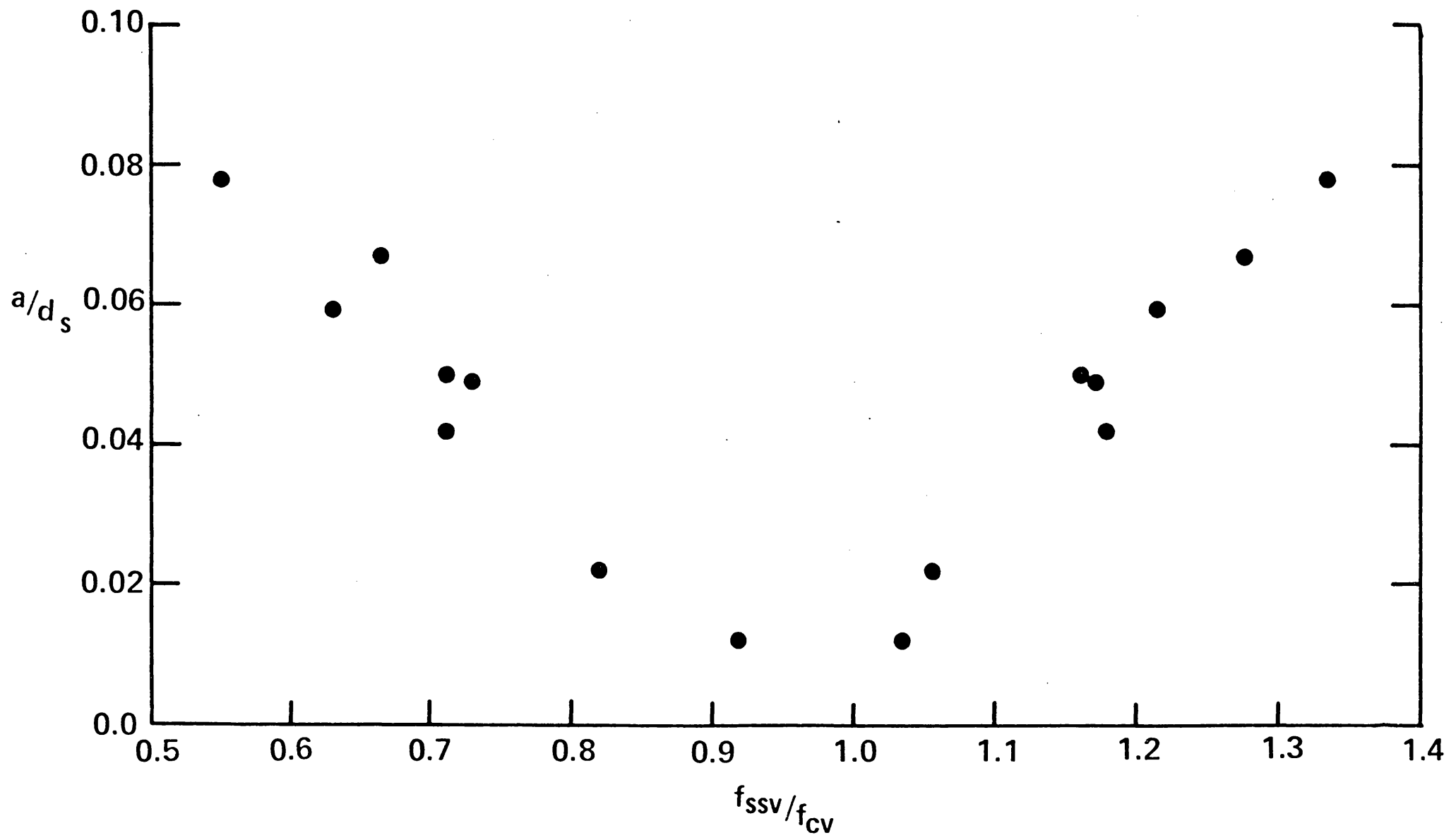

Figure 22. Sphere Lock-on Regions as a Function of Vibration Amplitude a/ds. 


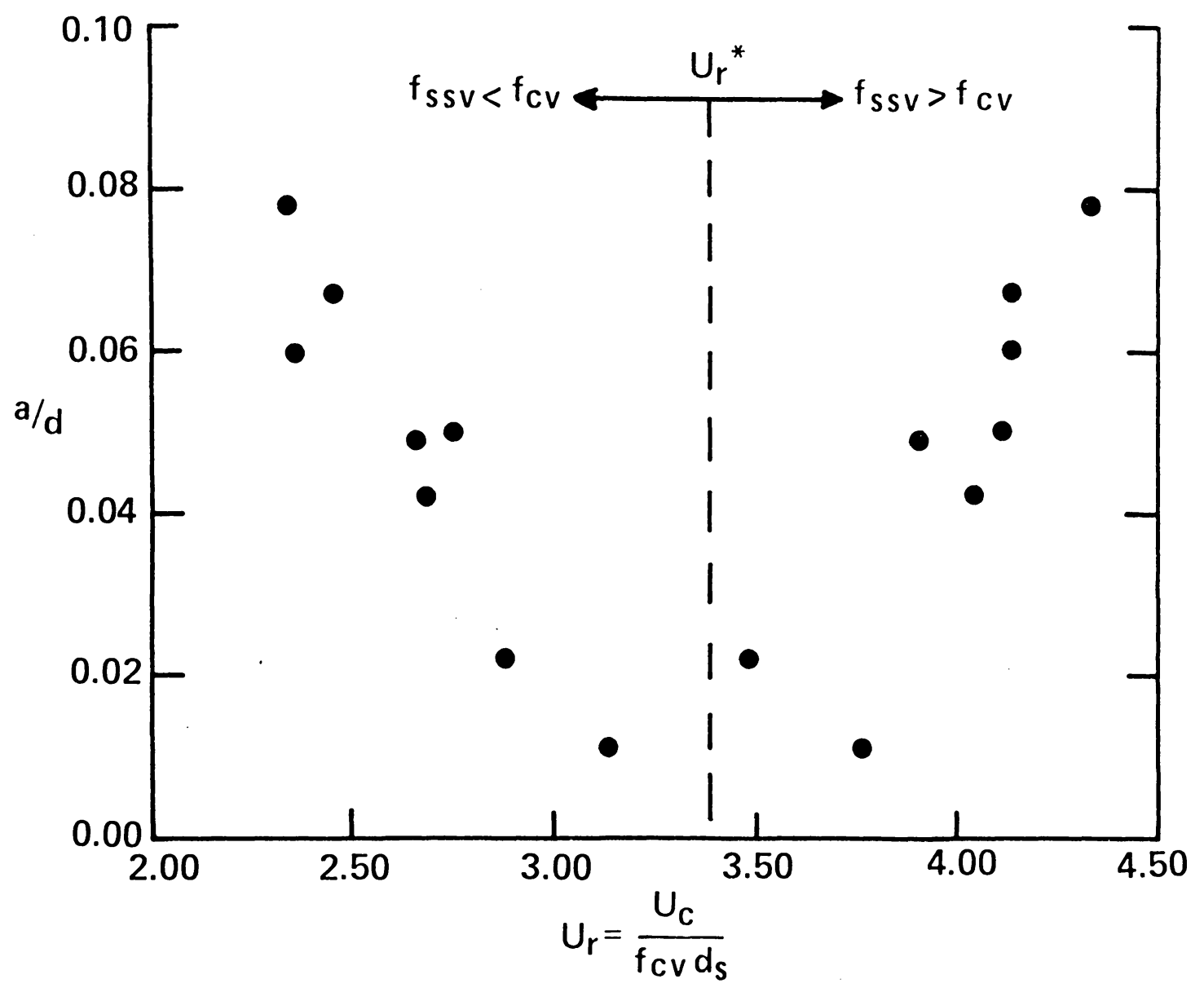

Figure 23. Reduced Velocity at the Boundaries of the Lockon Region for the Vibrating Sphere as a Function of the Vibration Amplitude. 


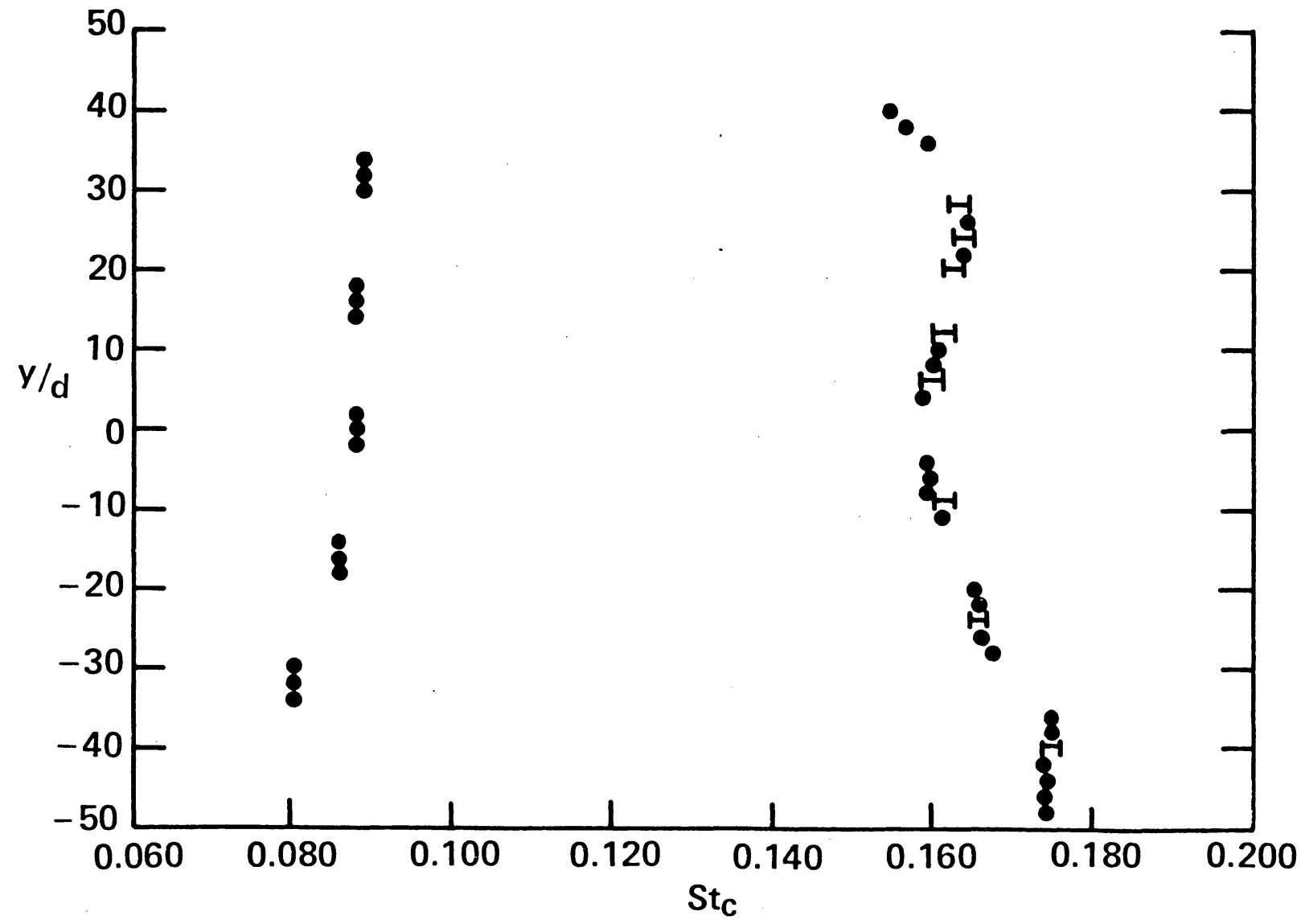

Figure 24. Spanise St rouhal Number Variation Along the Vibrating Cable with Five Spheres in Unsheared $\mathrm{Flow}, \mathrm{a} / \mathrm{d}=24.2 \%$, First $\mathrm{Mbde}, \operatorname{Re}_{\mathrm{c}}=2.23 \times 10^{3}$, Away from Lock-on Range. 

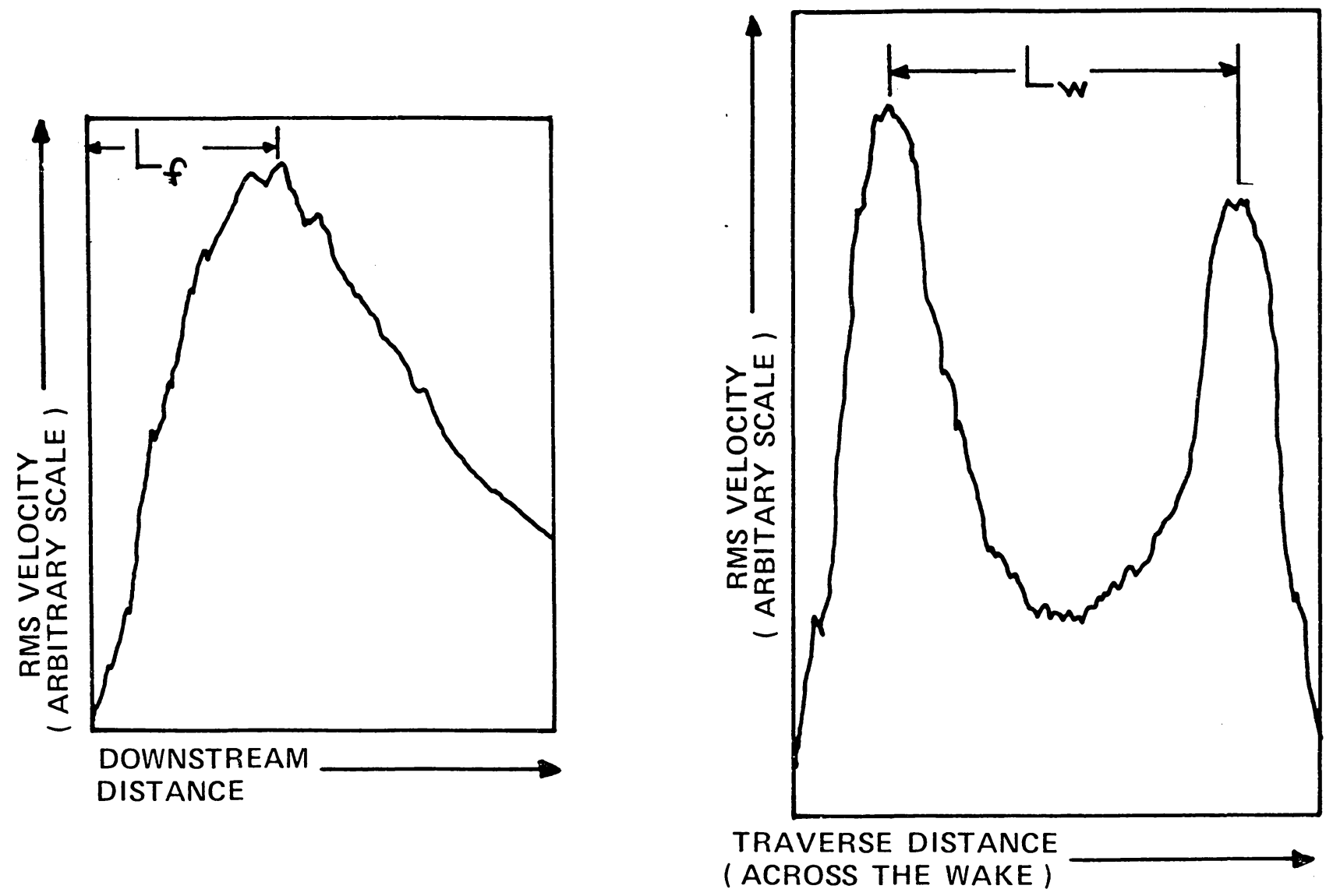

Figure 25. Typical Formation Length and Wake Width Traces. 

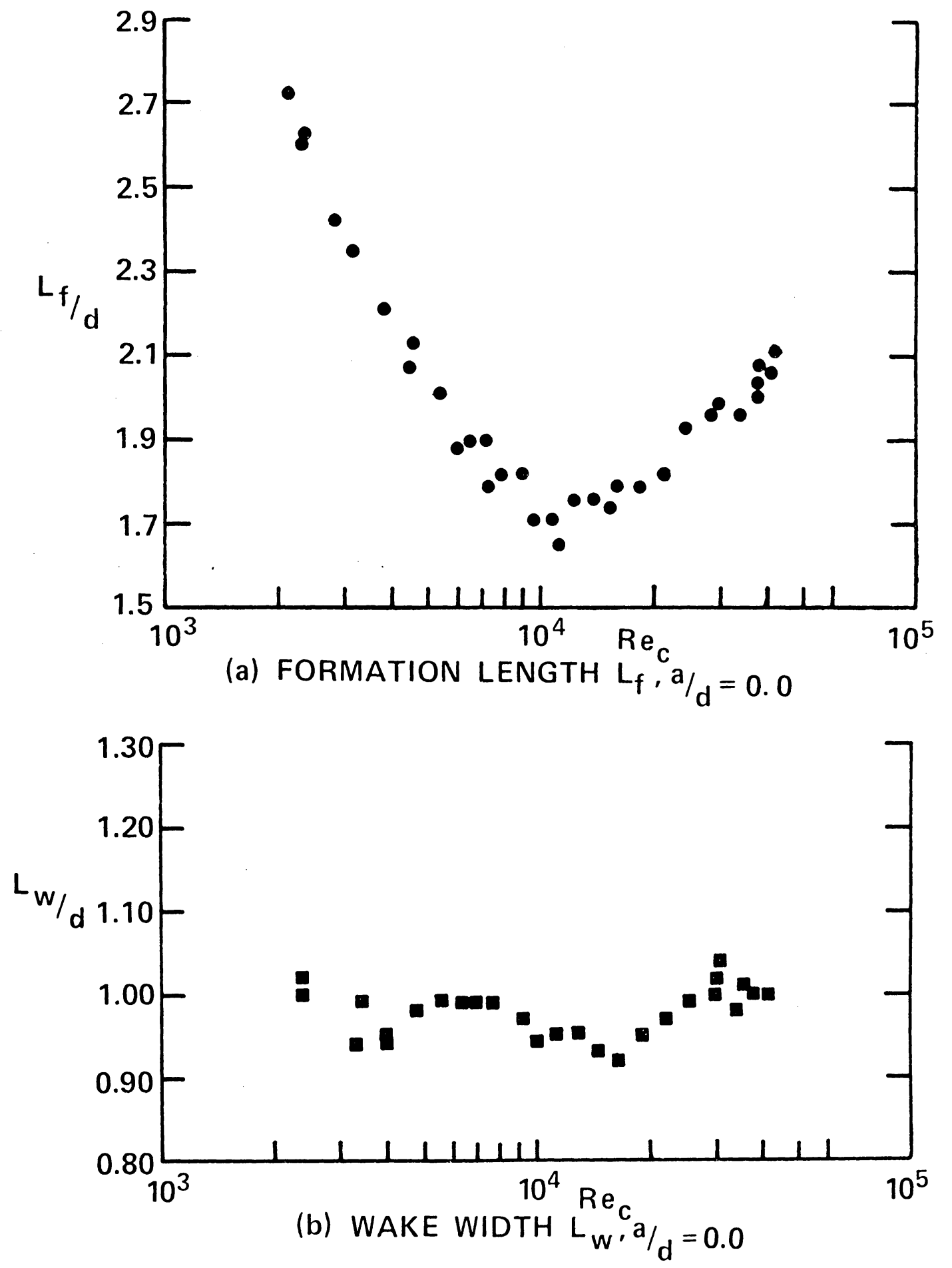

Figure 26. Variation of $\mathrm{L}_{\mathrm{f}}$ and $\mathrm{L}_{\mathrm{w}}$ as a Function of Increasing Reynolds Number. 


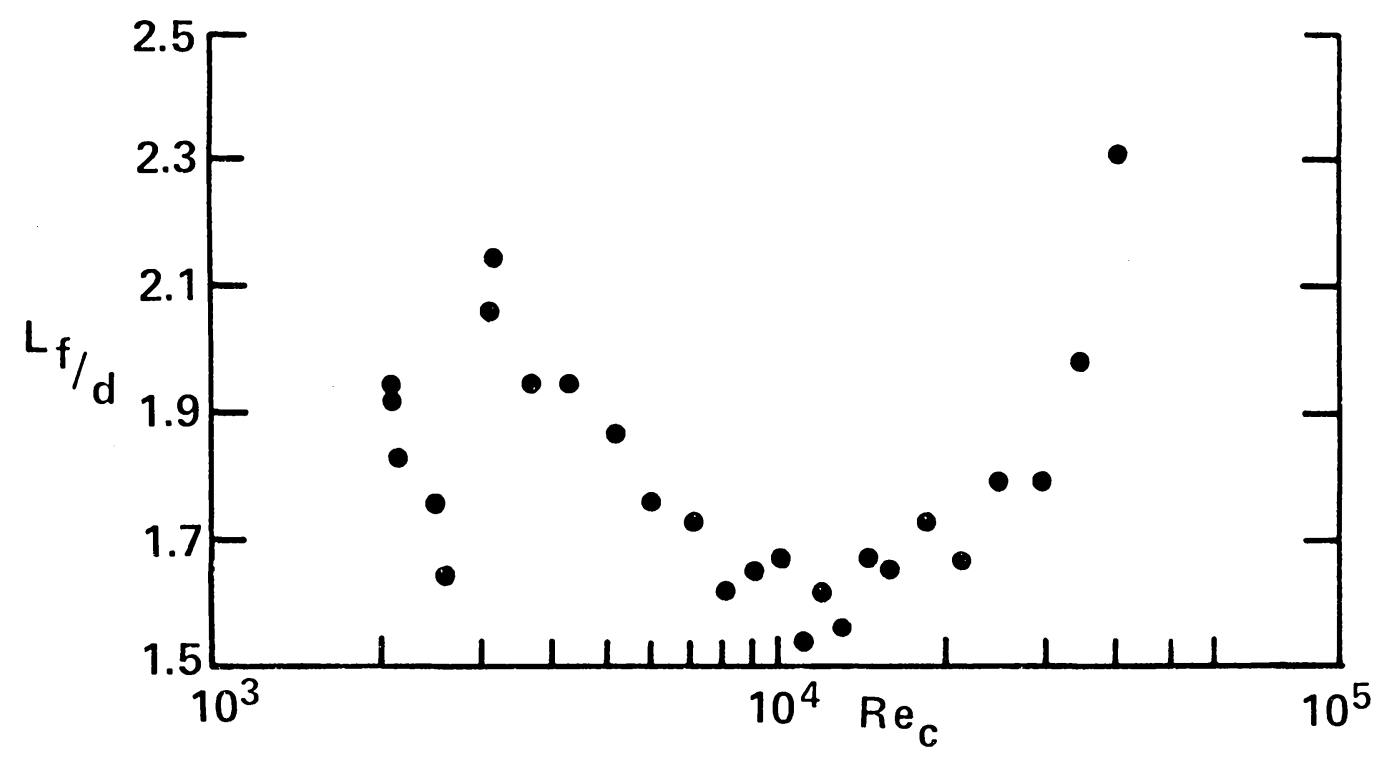

(a) FORMATION LENGTH $L_{f}, a / d=9.9 \%$

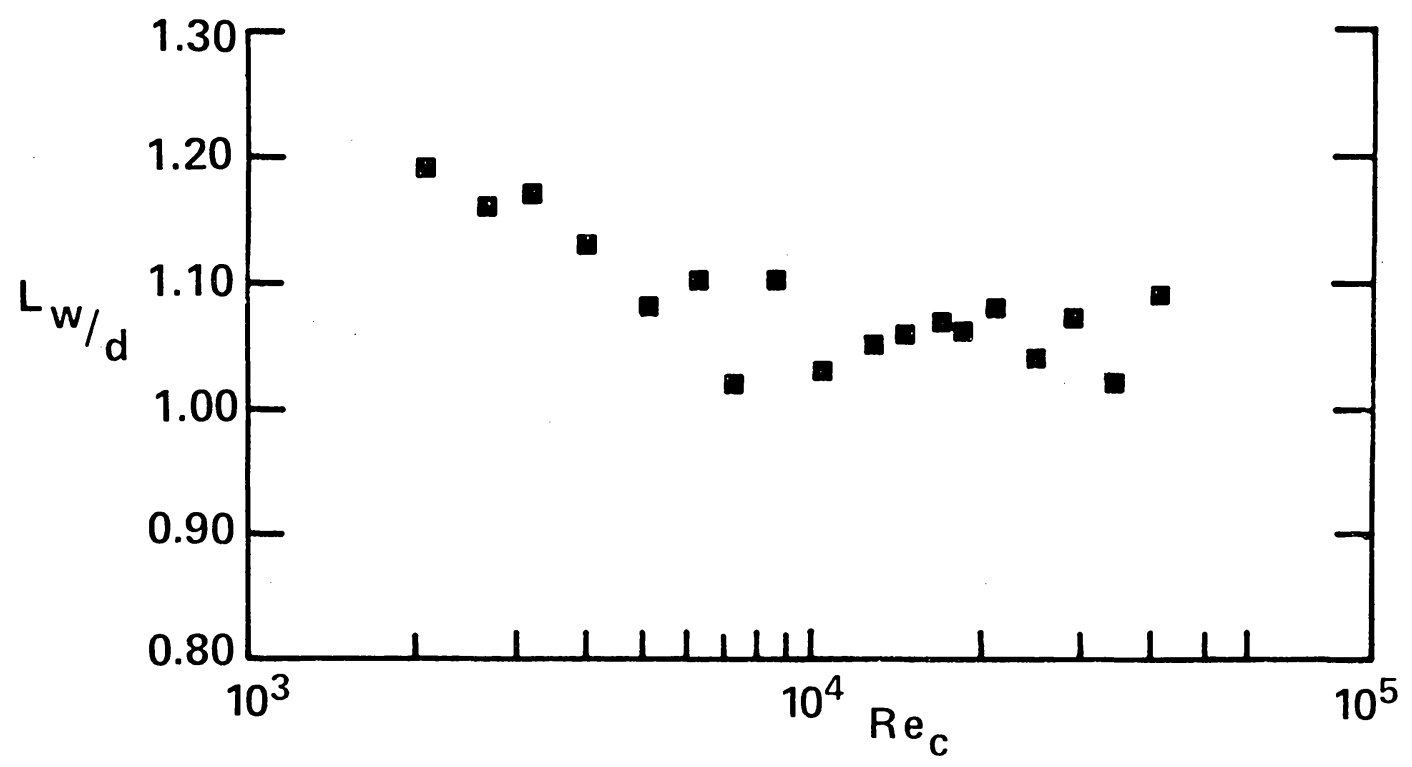

(b) WAKE WIDTH $L_{w}, a / d=9.9 \%$

Figure 27. Variation of $L_{f}$ and $L_{w}$ as a Function of Increasing Reynolds Number. 


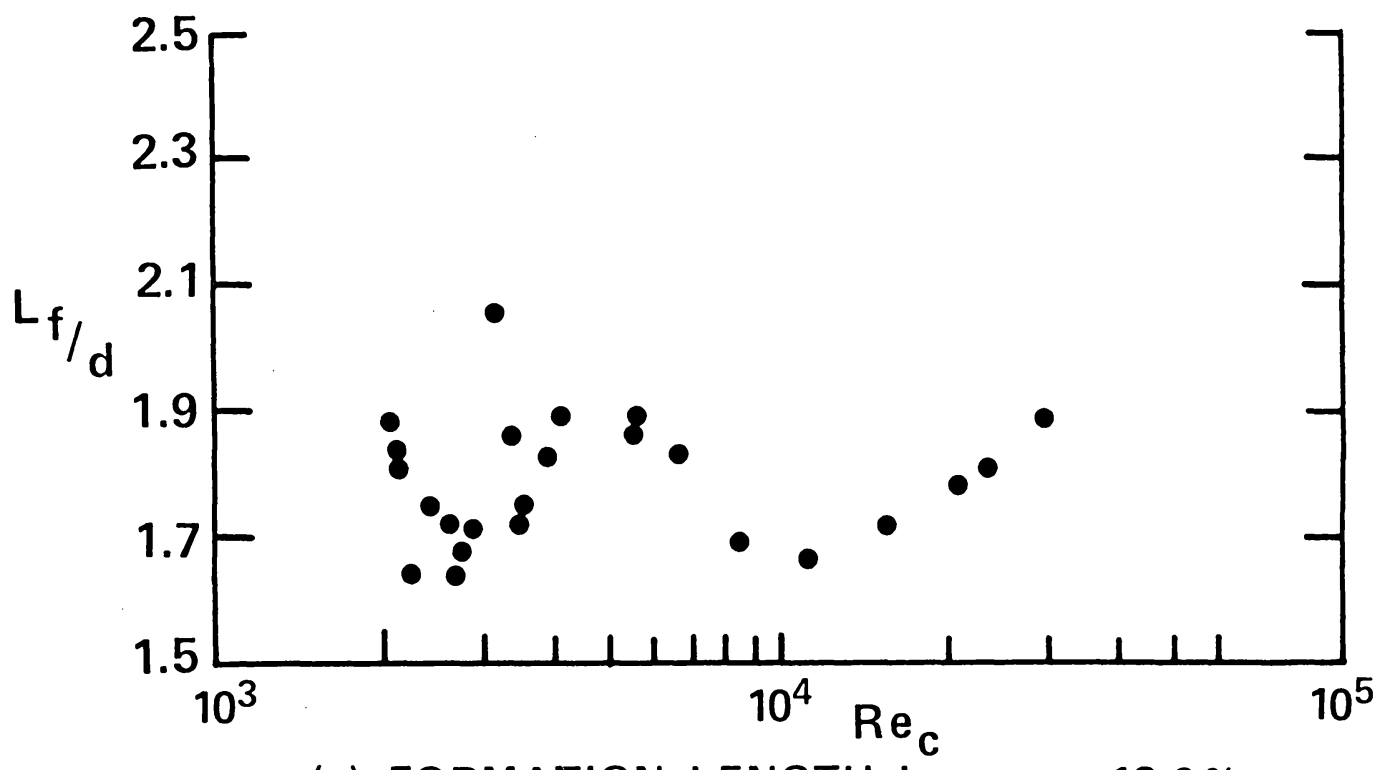

(a) FORMATION LENGTH $L_{f}, a / d=13.0 \%$

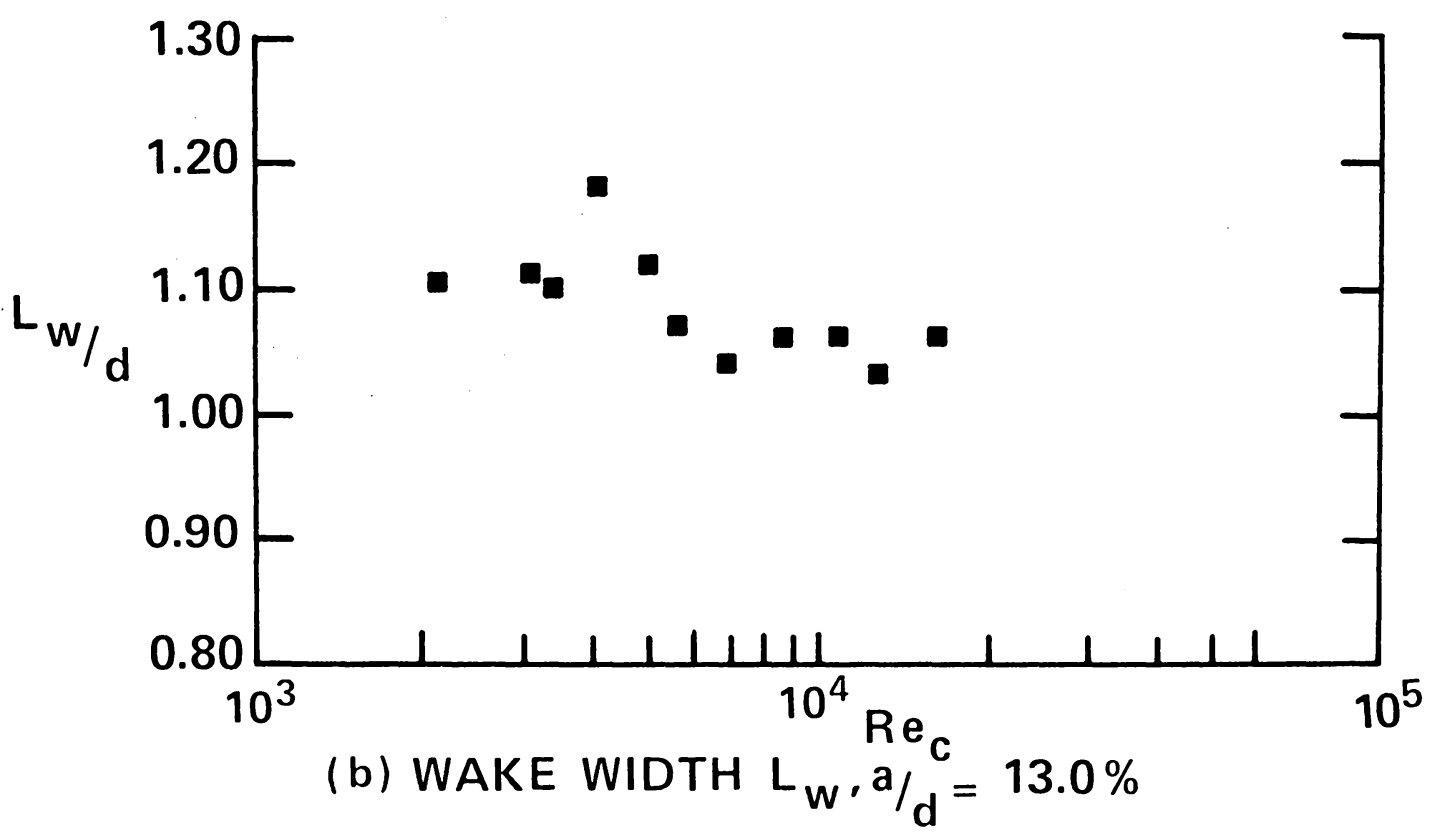

Figure 28. Variation of $\mathrm{L}_{\mathrm{f}}$ and $\mathrm{L}_{\mathrm{w}}$ as a Function of Increasing Reynolds Number. 


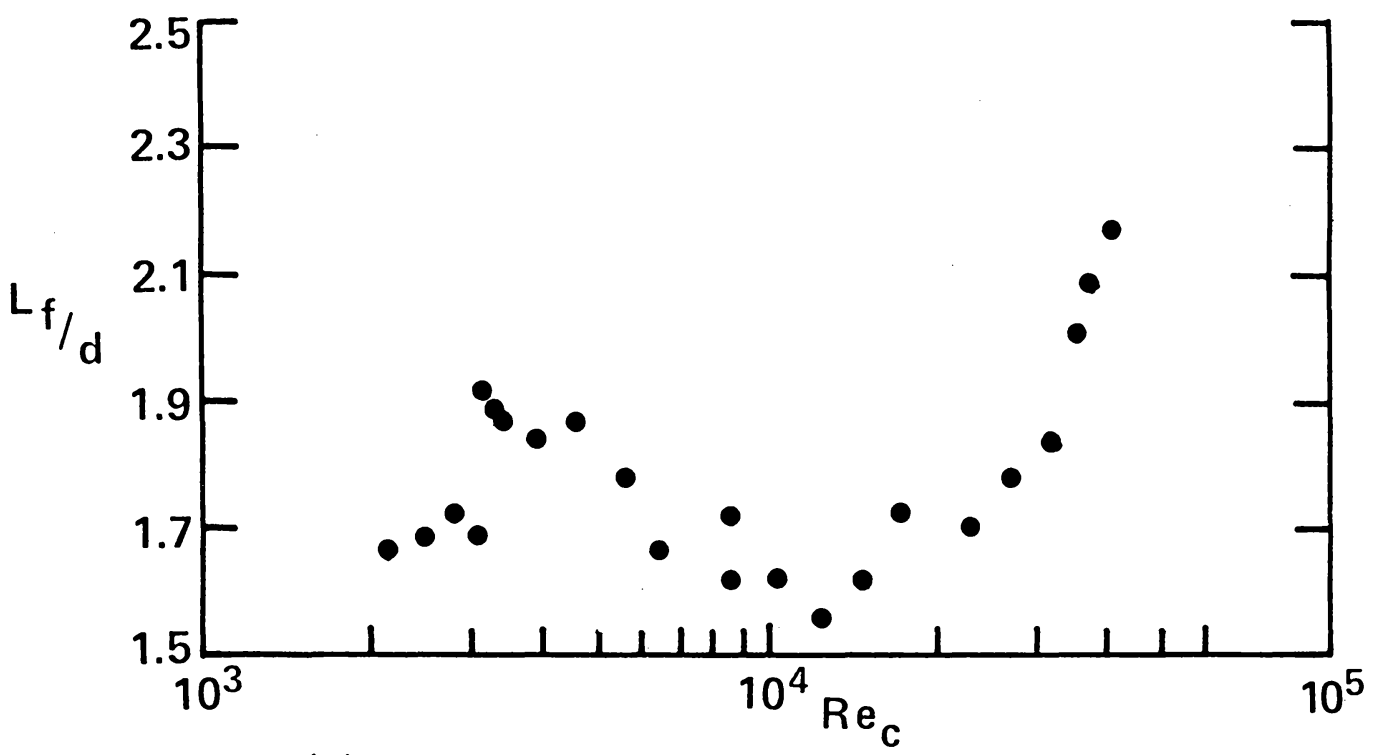

(a) FORMATION LENGTH $L_{f}, a / d=17.6 \%$

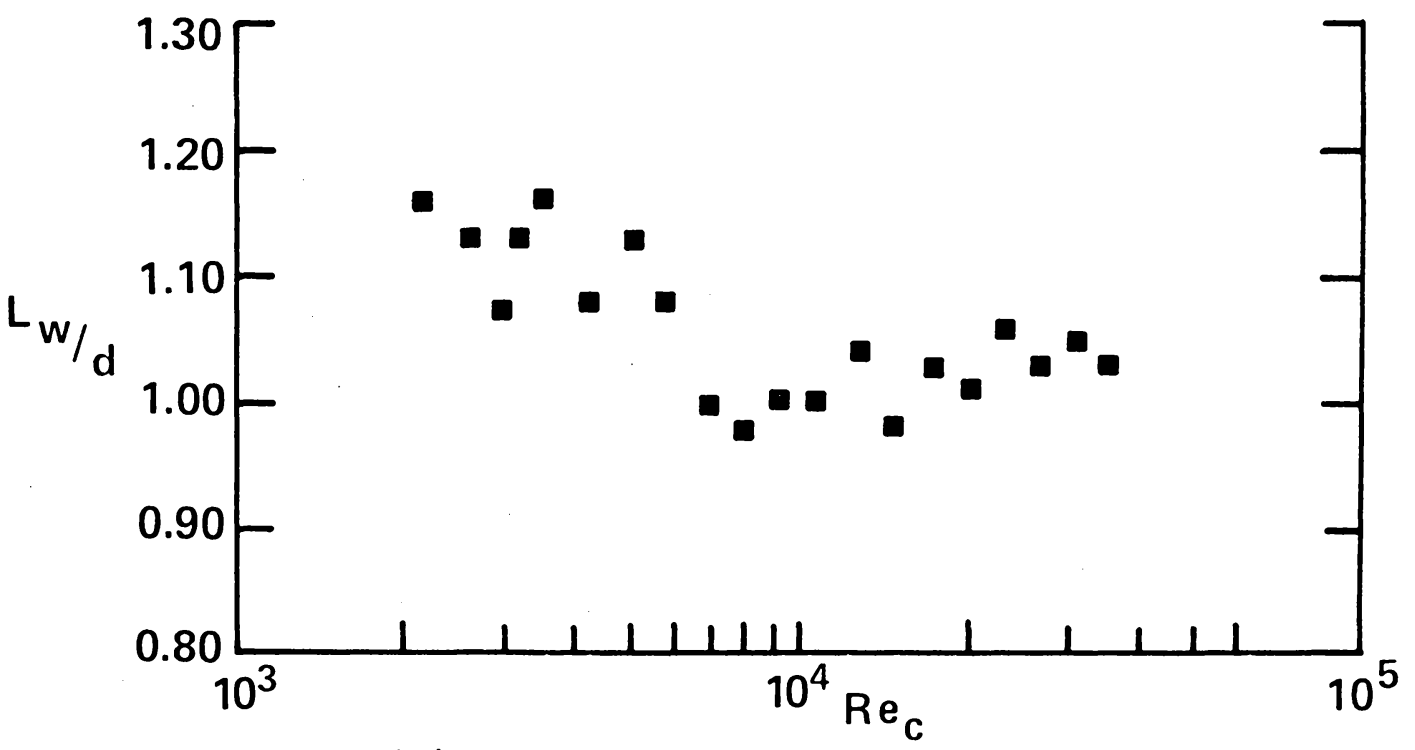

(b) WAKE WIDTH $L_{w}, a / d=19.8 \%$

Figure 29. Variation of $\mathrm{L}_{\mathrm{f}}$ and $\mathrm{L}_{\mathrm{w}}$ as a Function of Increasing Reynolds Number. 


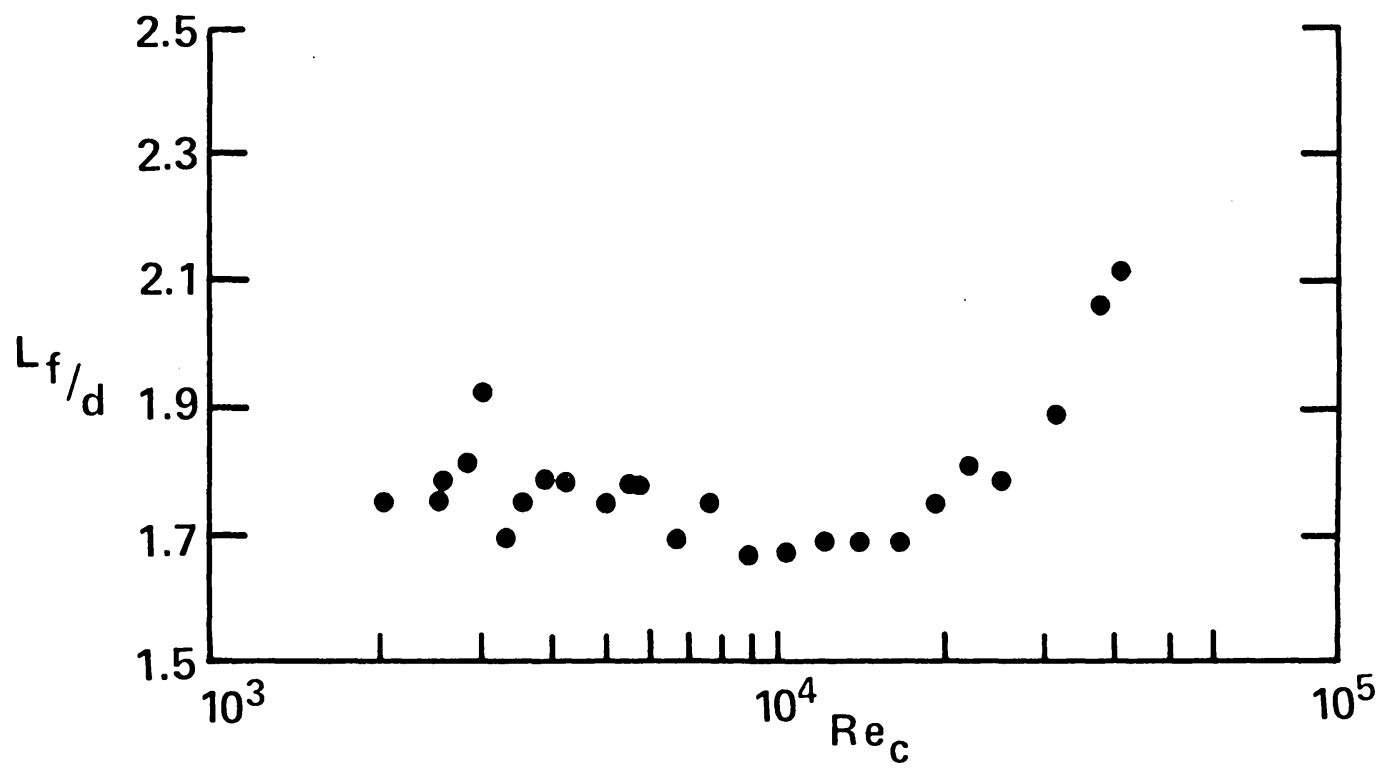

(a) FORMATION LENGTH $L_{f}, a / d=25.5 \%$

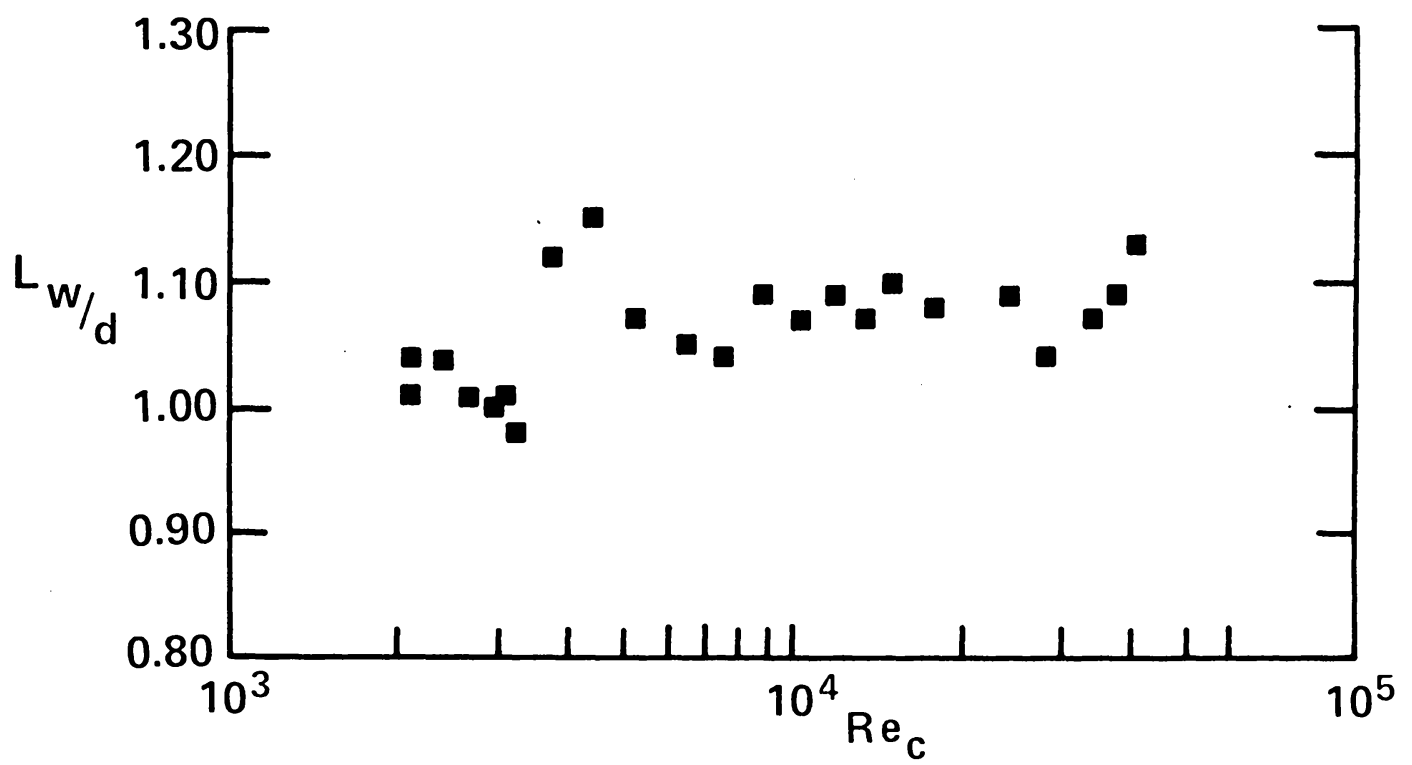

(b) WAKE WIDTH $L_{w}, a / d=25.5 \%$

Figure 30. Variation of $L_{f}$ and $L_{w}$ as a Function of Increasing Reynolds Number. 

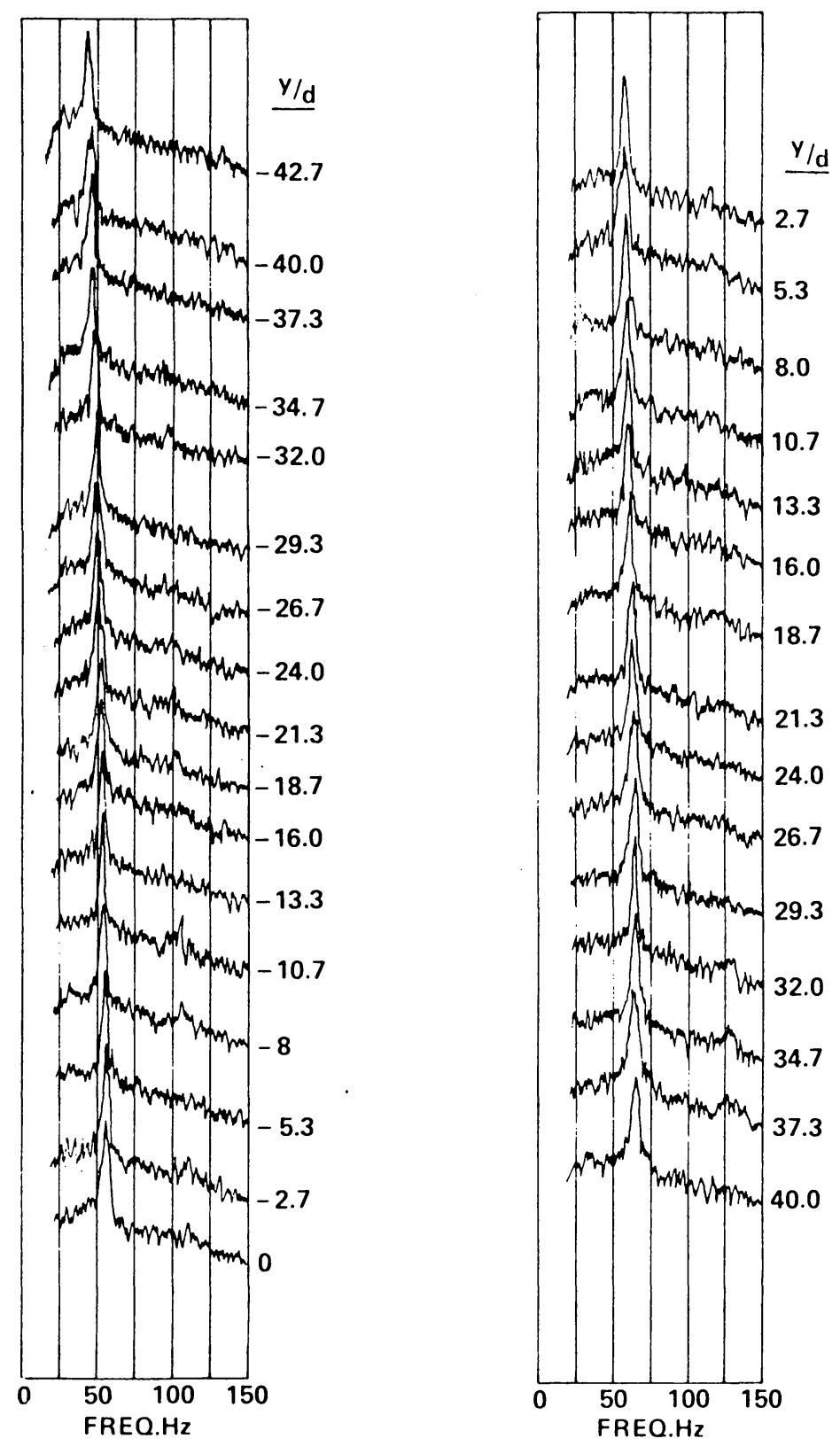

Figure 31. Power Spectra Showing the Spanwise Variation of the Vortex Shedding Frequency, First Phase Test, $\operatorname{Re}_{c}=2.6 \times 10^{3}$, No Vibration, No Spheres, $\beta=0.0053$. 


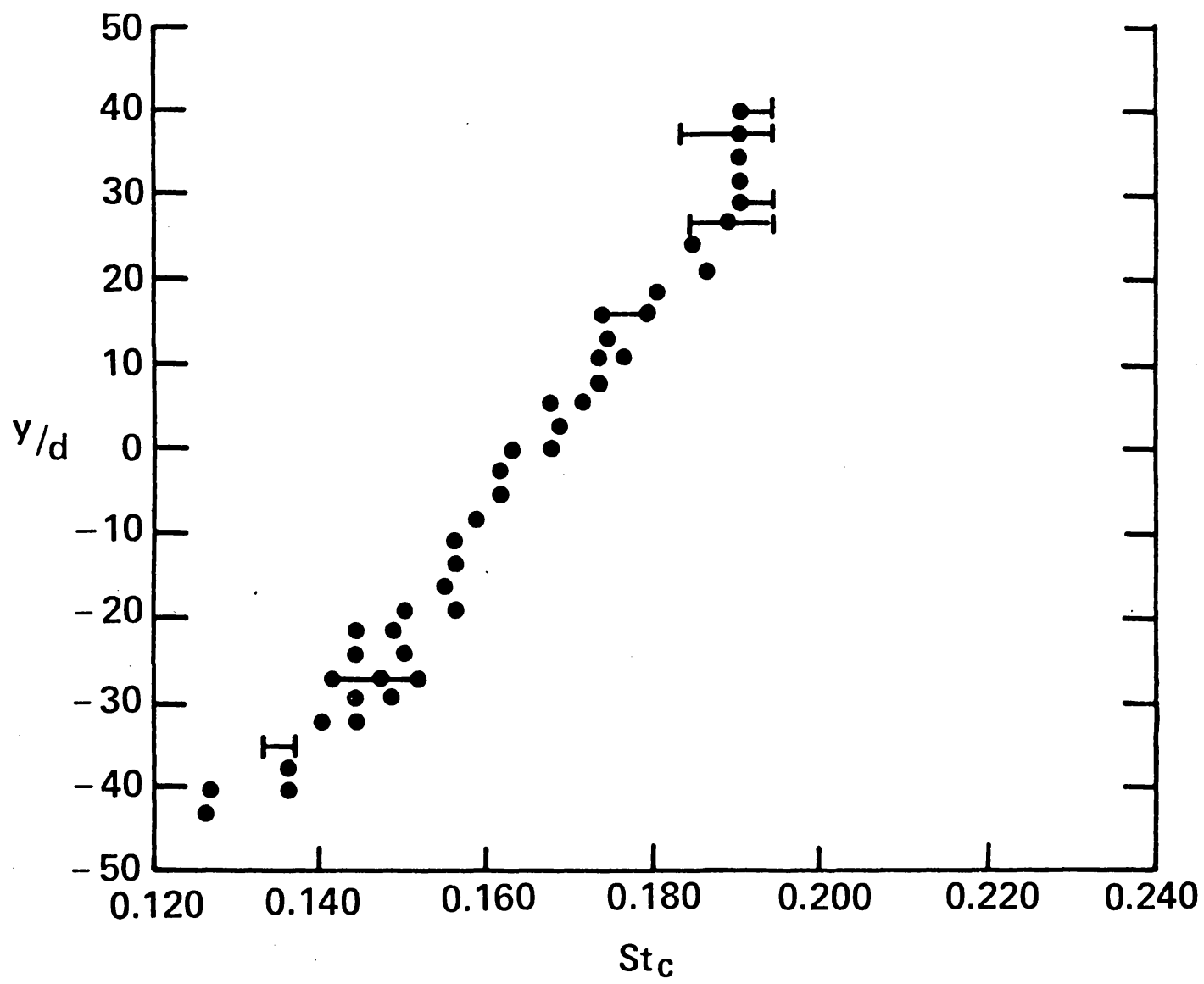

Figure 32. Spanwise St rouhal Number Variation, First Phase Test, $\operatorname{Re}_{c}=2.6 \times 10^{3}$, No Vibration, No Spheres, $B=0.0053$. 

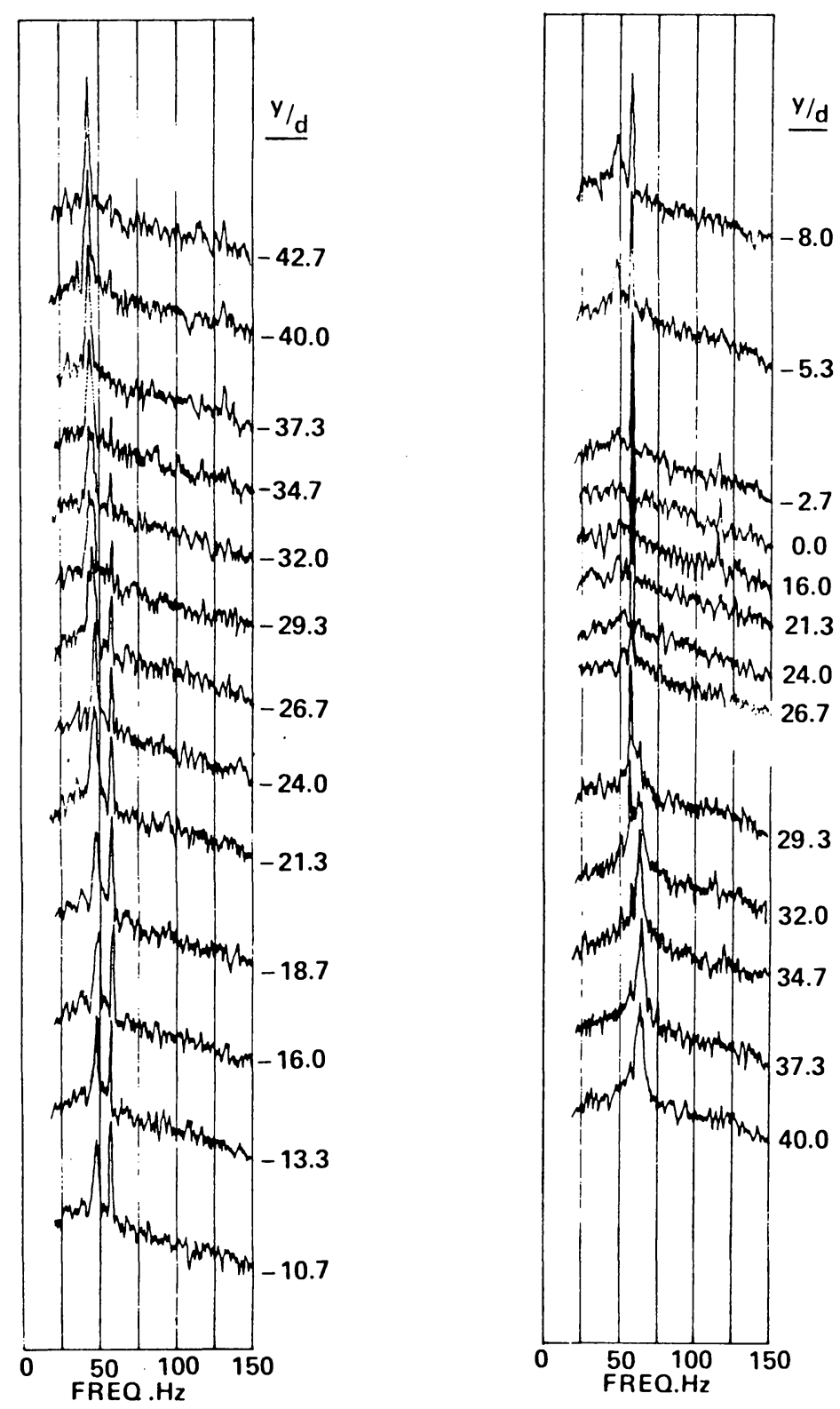

Figure 33. Power Spectra Showing the Spanwise Variation of the Vortex Shedding Frequency, First Phase Test, $\operatorname{Re}_{c}=2.6 \times 10^{3}$, First Mode Vibration, $a / d=23.3 \%$, No Spheres, $B=0.0053$. 


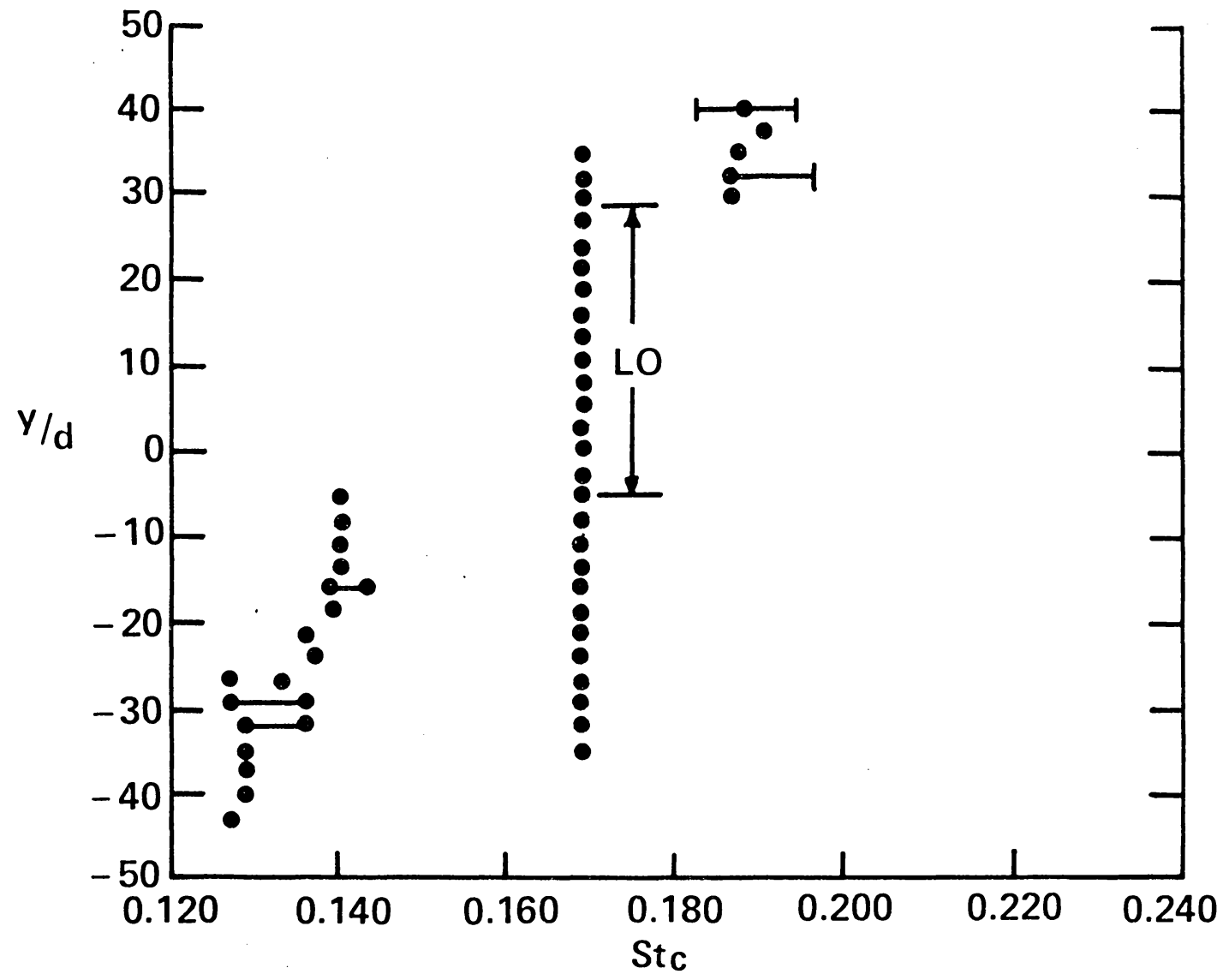

Figure 34. Spanwise Strouhal Number Variation, First Phase Test, $\operatorname{Re}_{c}=2.6 \times 10^{3}$, First Mode Vibration, $\mathrm{a} / \mathrm{d}=23.3 \%$, No Spheres, $B=0.0053$. 

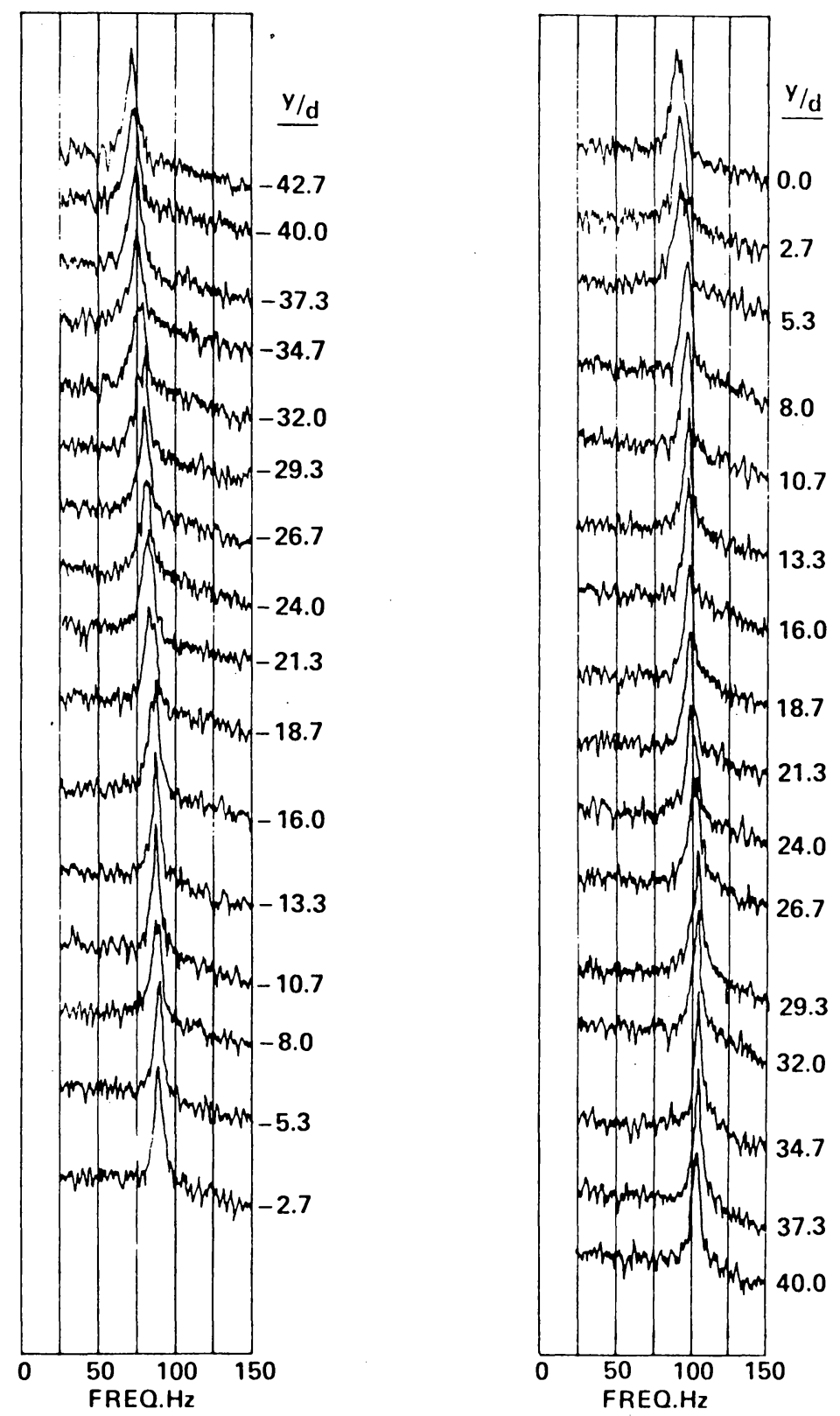

Figure 35. Power Spectra Showing the Spanwise Variation of the Vortex Shedding Frequency, First Phase Test, $\operatorname{Re}_{c}=3.72 \times 10^{3}$, No Vibration, No Spheres, $B=0.0053$. 


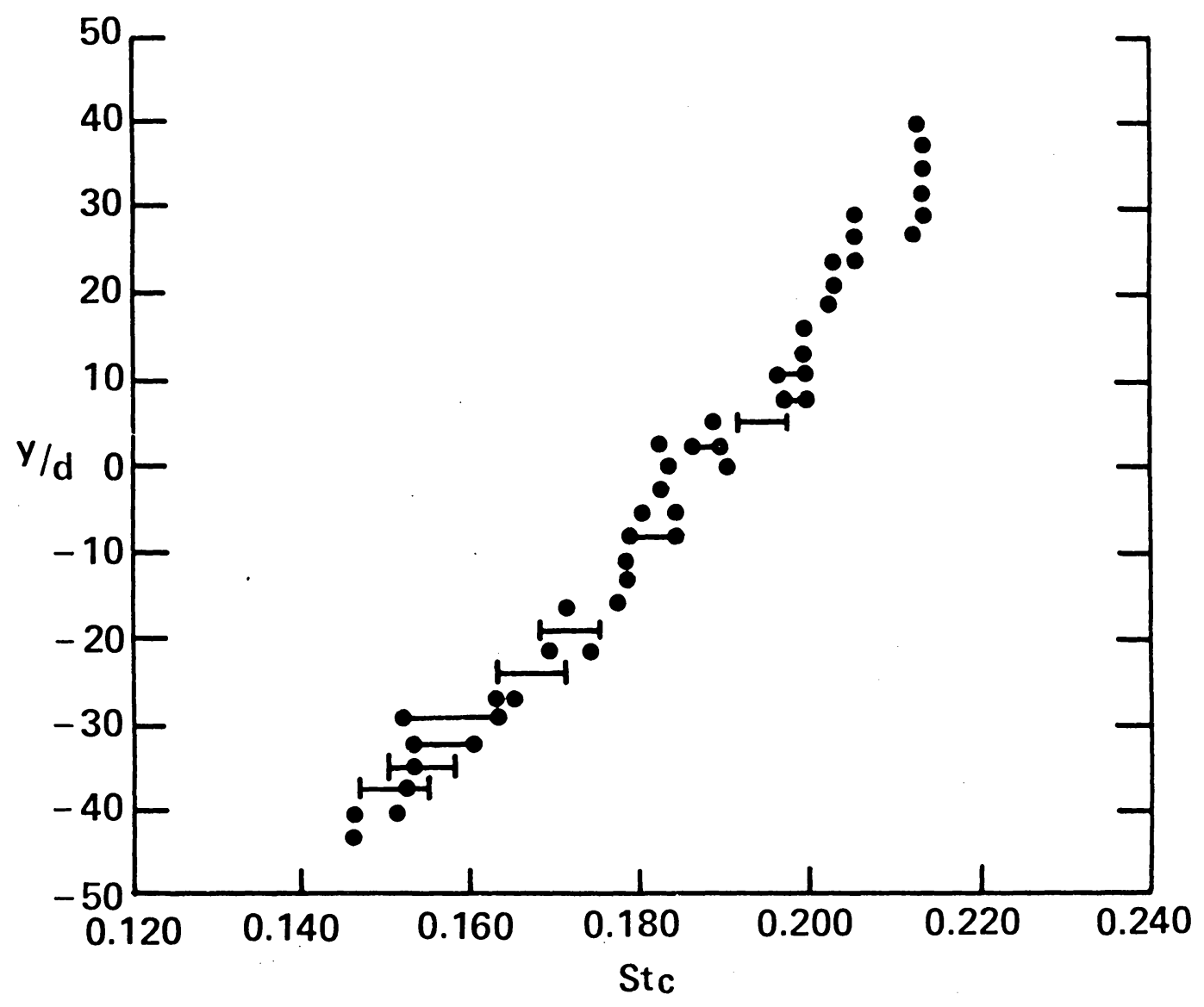

Figure 36. Spanwise St rouhal Number Variation, First Phase Test, $\operatorname{Re}_{c}=3.72 \times 10^{3}$, No Vibration, No Spheres, $\beta=0.0053$. 

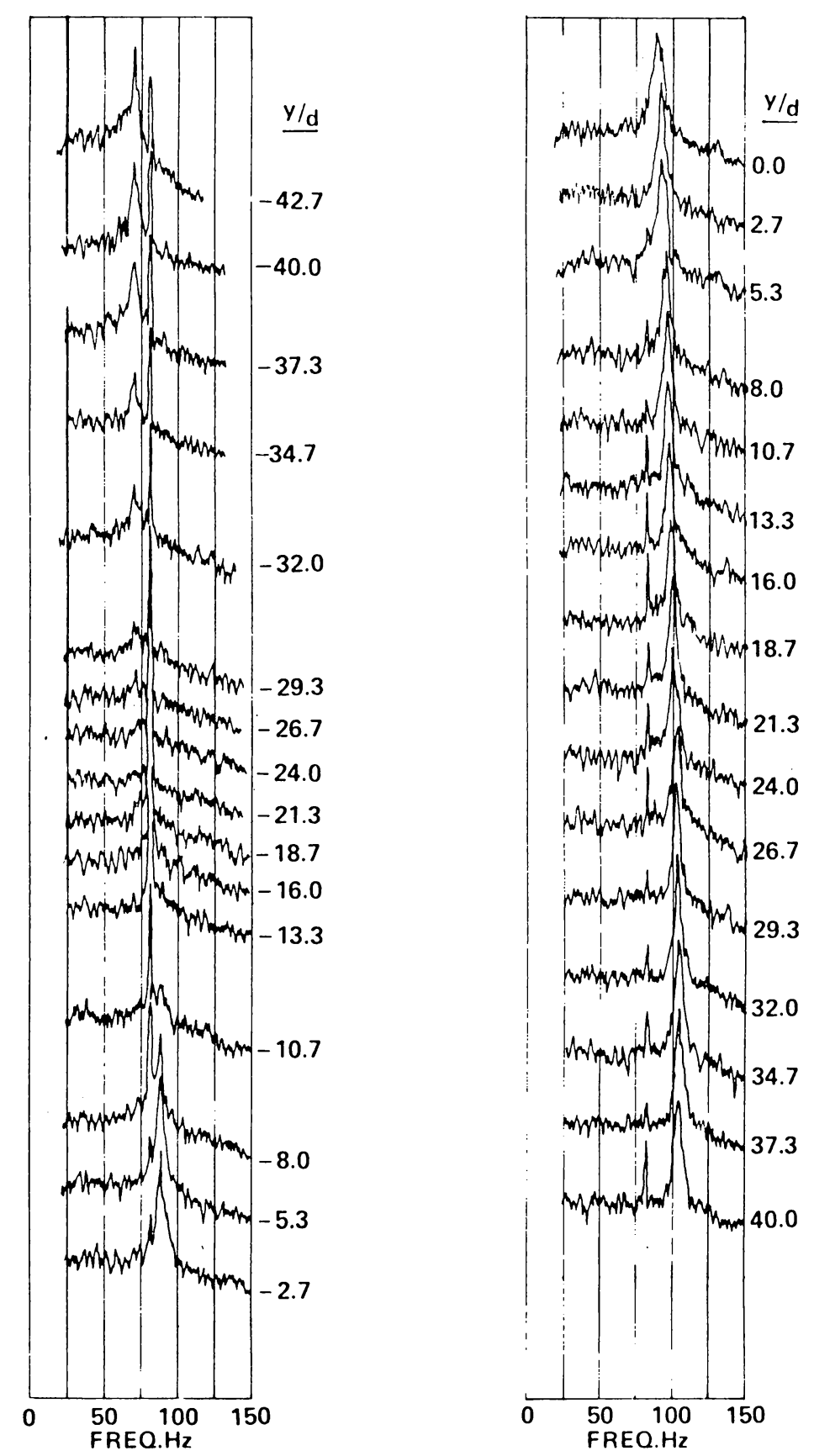

Figure 37. Power Spectra Showing the Spanwise Variation of the Vortex Shedding Frequency, First Phase Test, $\operatorname{Re}_{c}=3.72 \times 10^{3}$, Second Mode Vibration, $\mathrm{a} / \mathrm{d}=11.7 \%$, No Spheres, $\beta=0.0053$. 


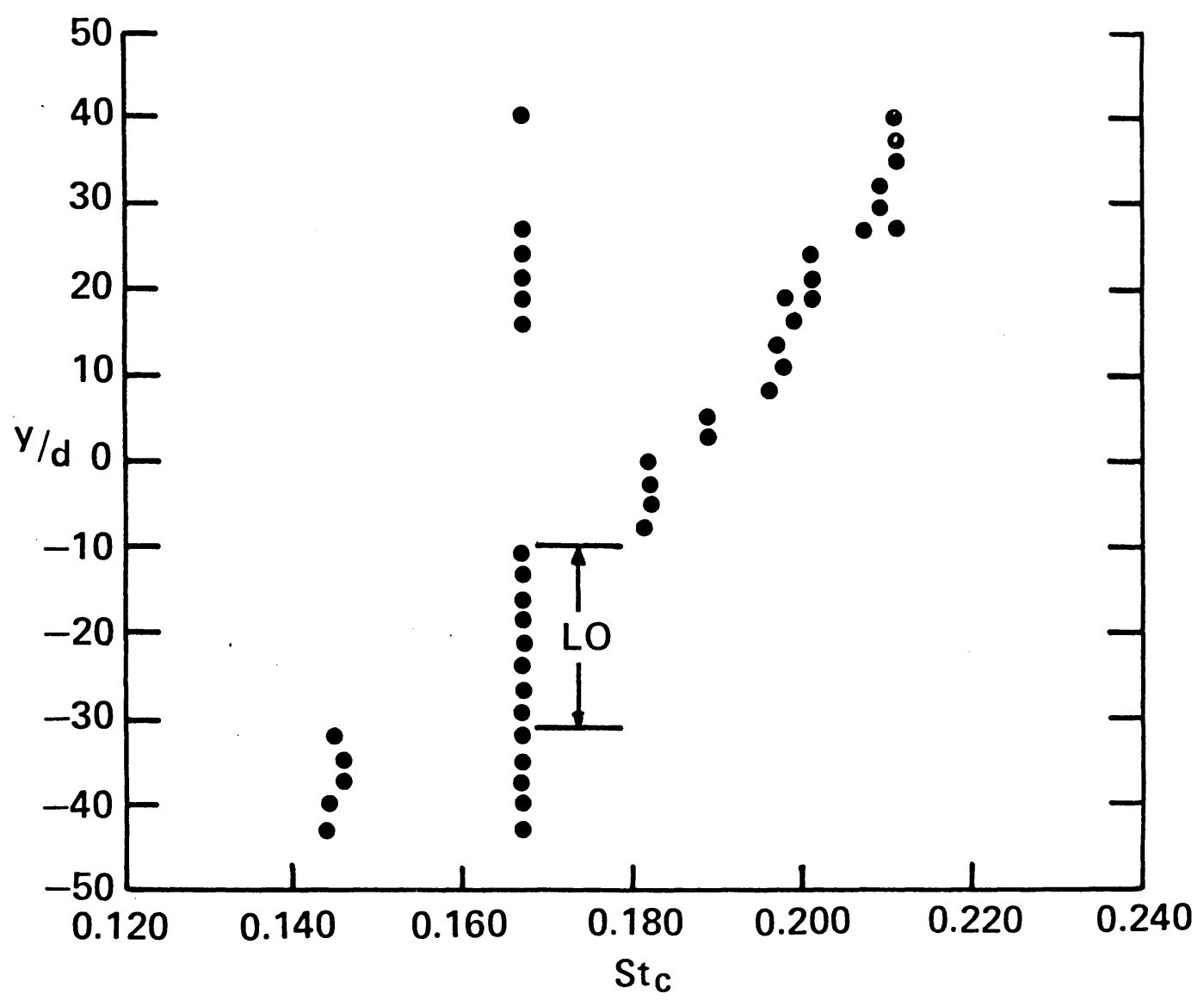

Figure 38. Spanwise Strouhal Number Variation, First Phase Test, $\operatorname{Re}_{c}=3.72 \times 10^{3}$, Second Mode Vibration, $\mathrm{a} / \mathrm{d}=11.7 \%$, No Spheres, $\beta=0.0053$. 

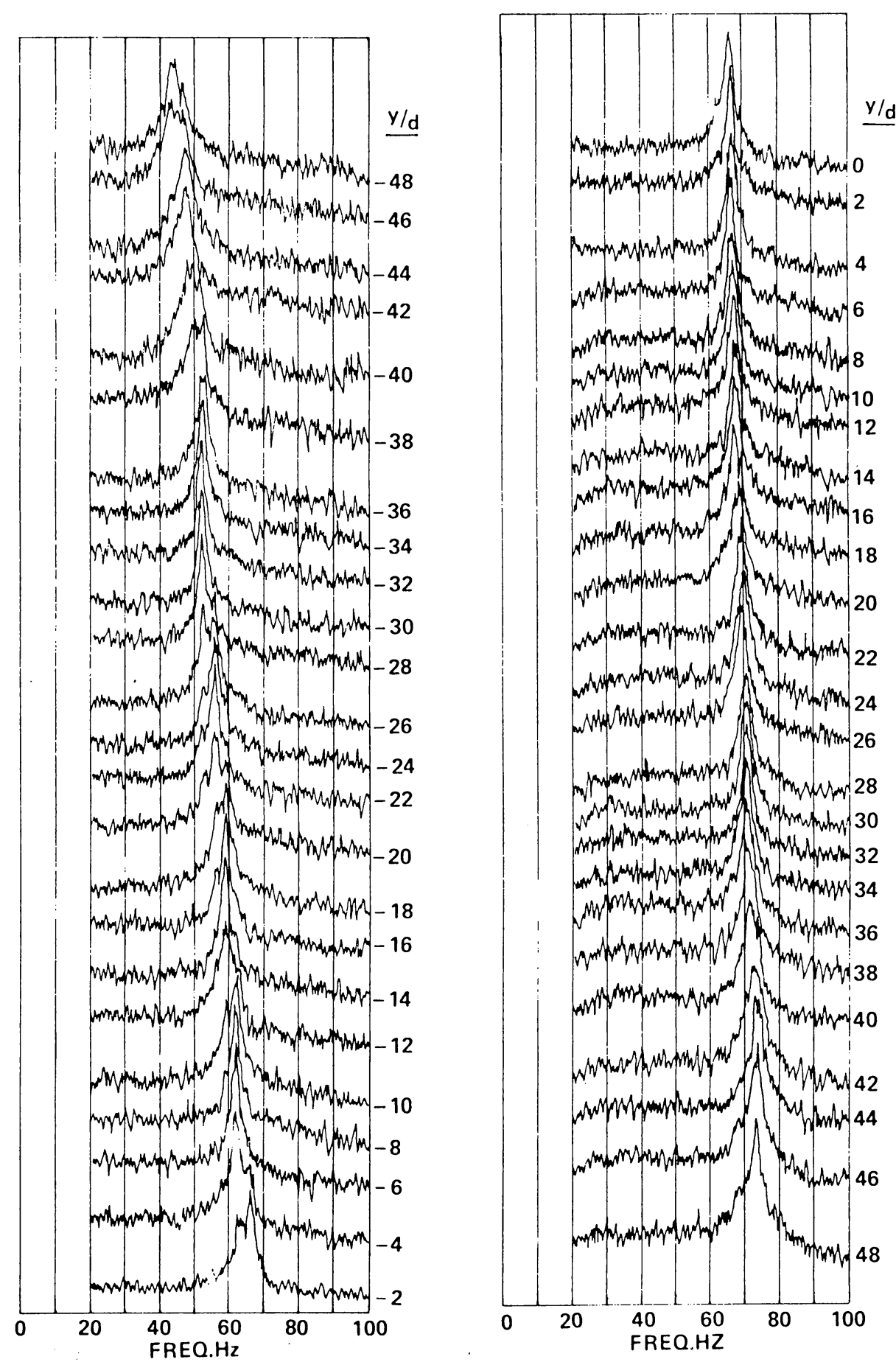

Figure 39. Power Spectra Showing the Spanwise Variation of the Vortex Shedding Frequency, $\operatorname{Re}_{c}=2.96 \mathrm{x}$ $10^{3}$, No Vibration, No Spheres, $\beta=0.0053$. 


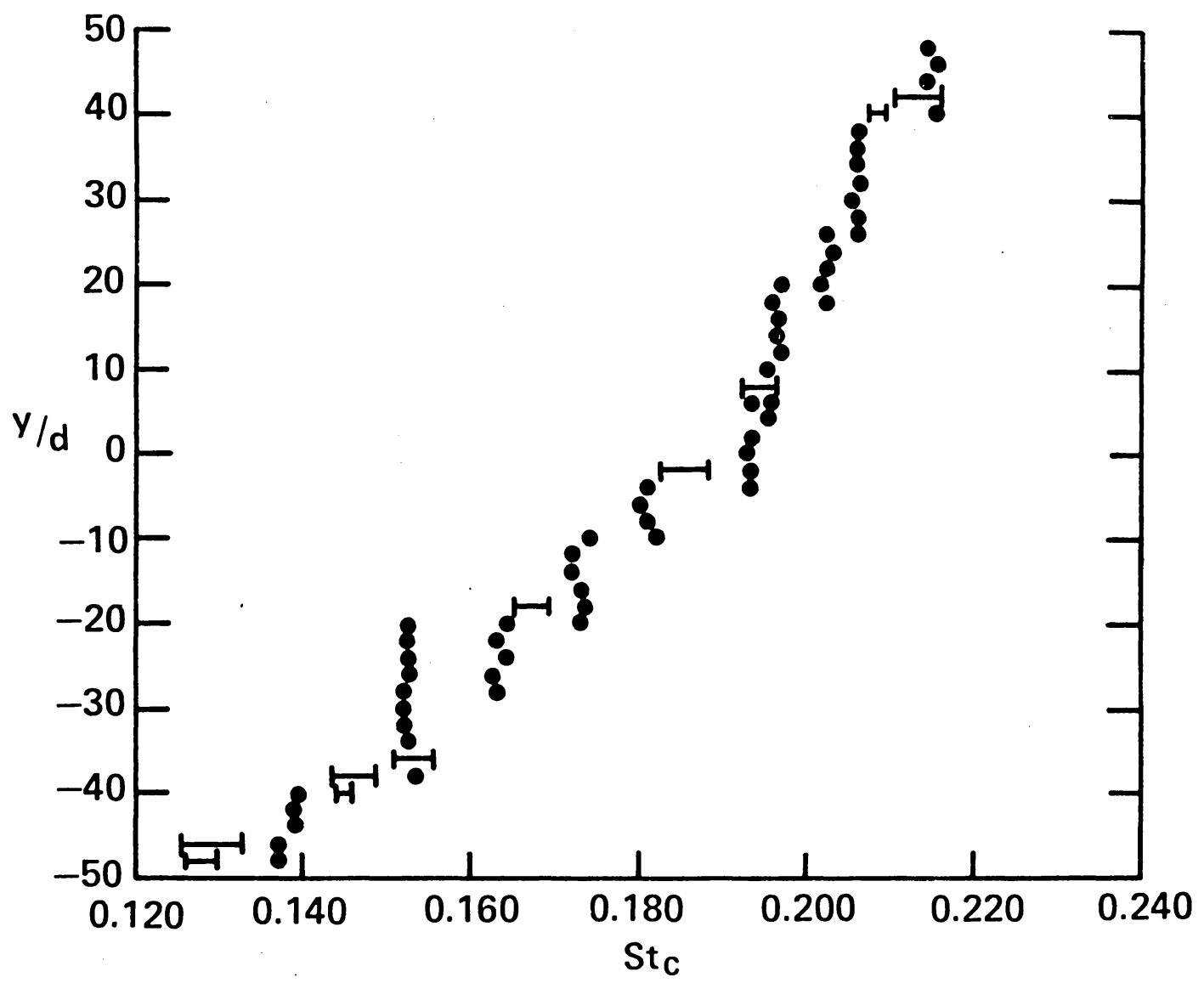

Figure 40. Spanwise Strouhal Number Variation, $\operatorname{Re}_{c}=2.96$ $x 10^{3}$, No Vibration, No Spheres, $\beta=0.0053$. 

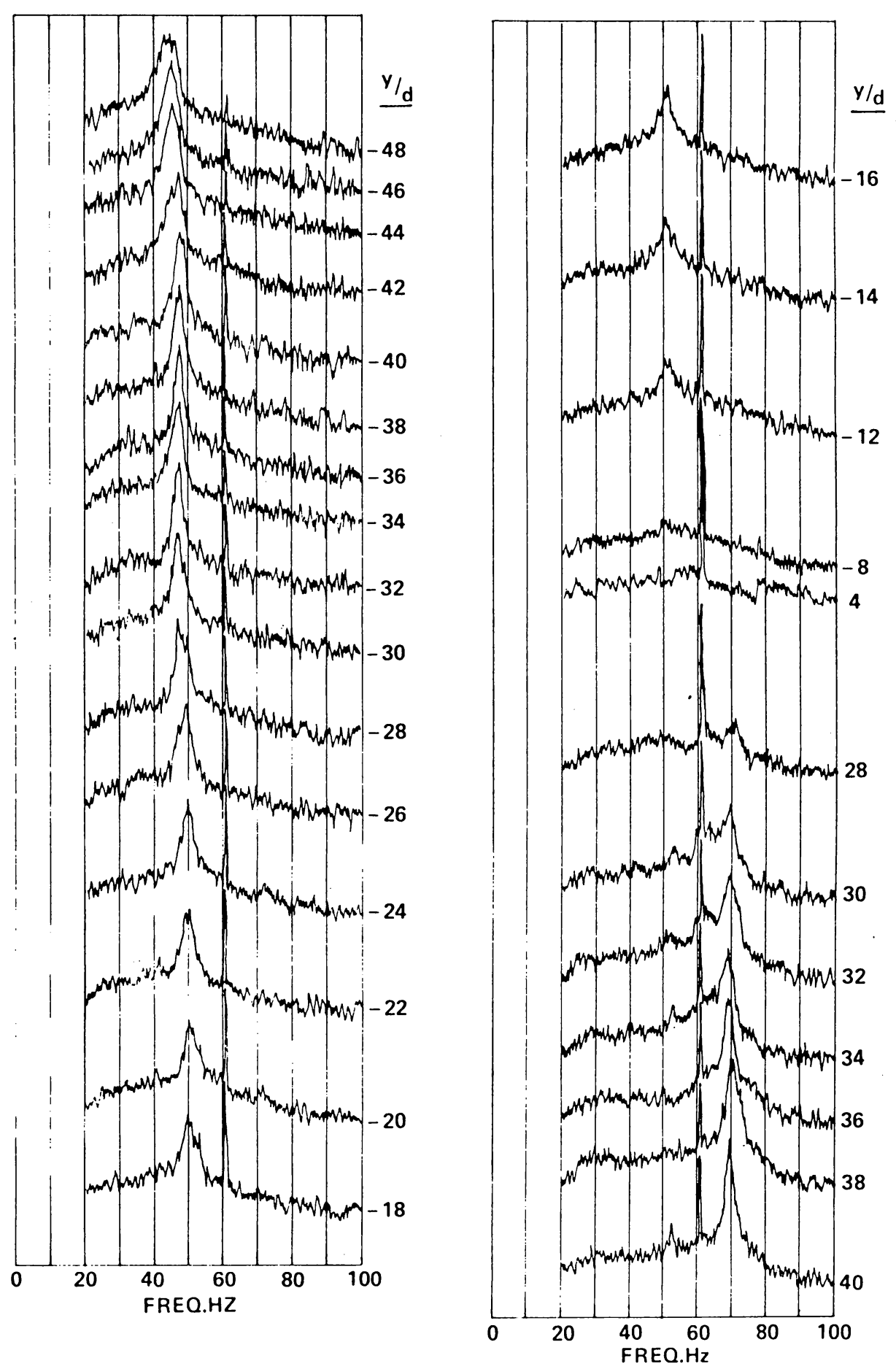

Figure 41. Power Spectra Showing the Spanwise Variation of the Vortex Shedding Frequency, $\operatorname{Re}_{c}=2.96 \mathrm{x}$ $10^{3}$, First Mode Vibration, aid $=29.1 \%$, No Spheres, $B=0.0053$. 


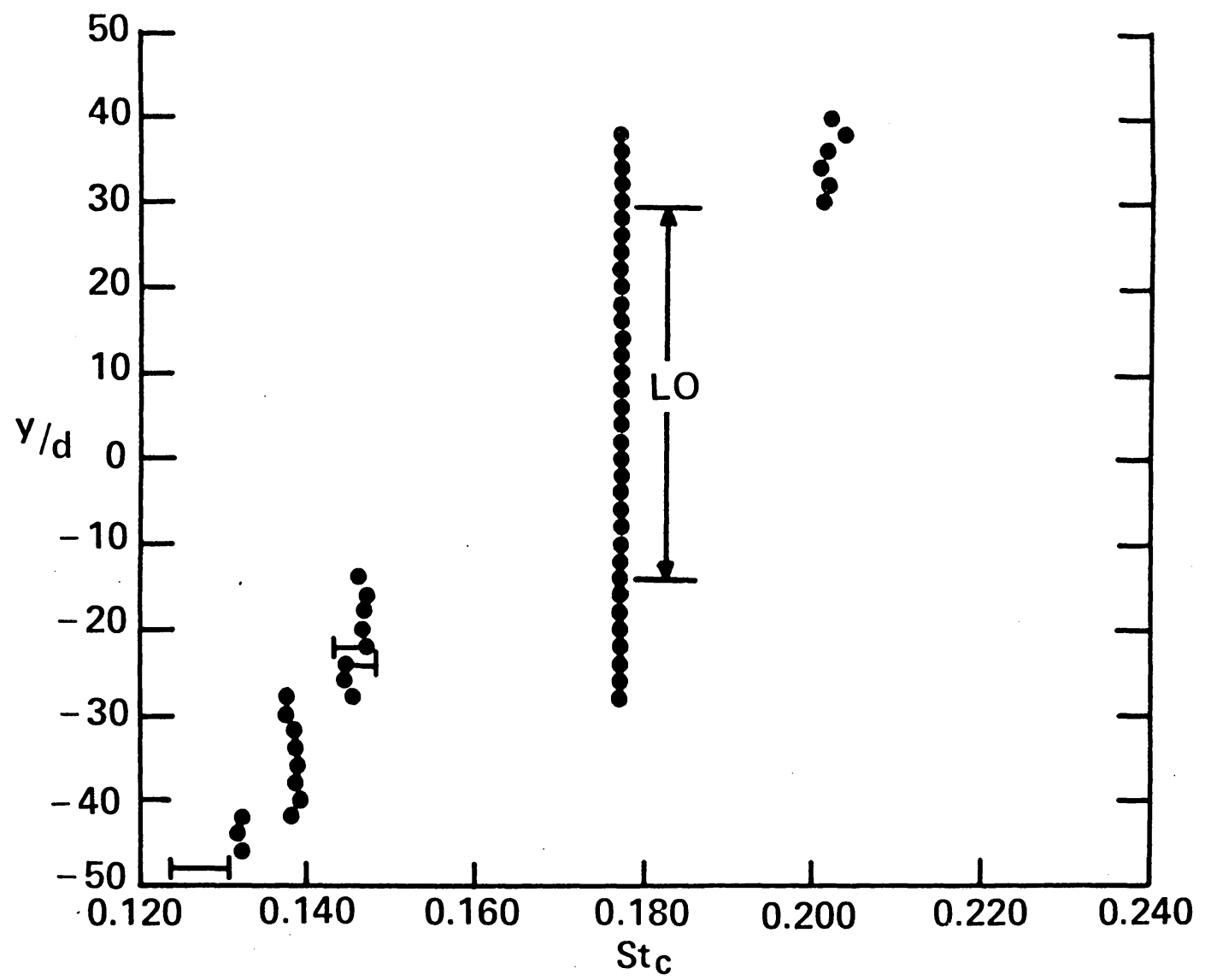

Figure 42. Spanwise St rouhal Number Variation, $\operatorname{Re}_{c}=2.96$ $x 10^{3}$, First Mode Vibration, a/d $=29.1 \%$, No Spheres, $B=0.0053$. 

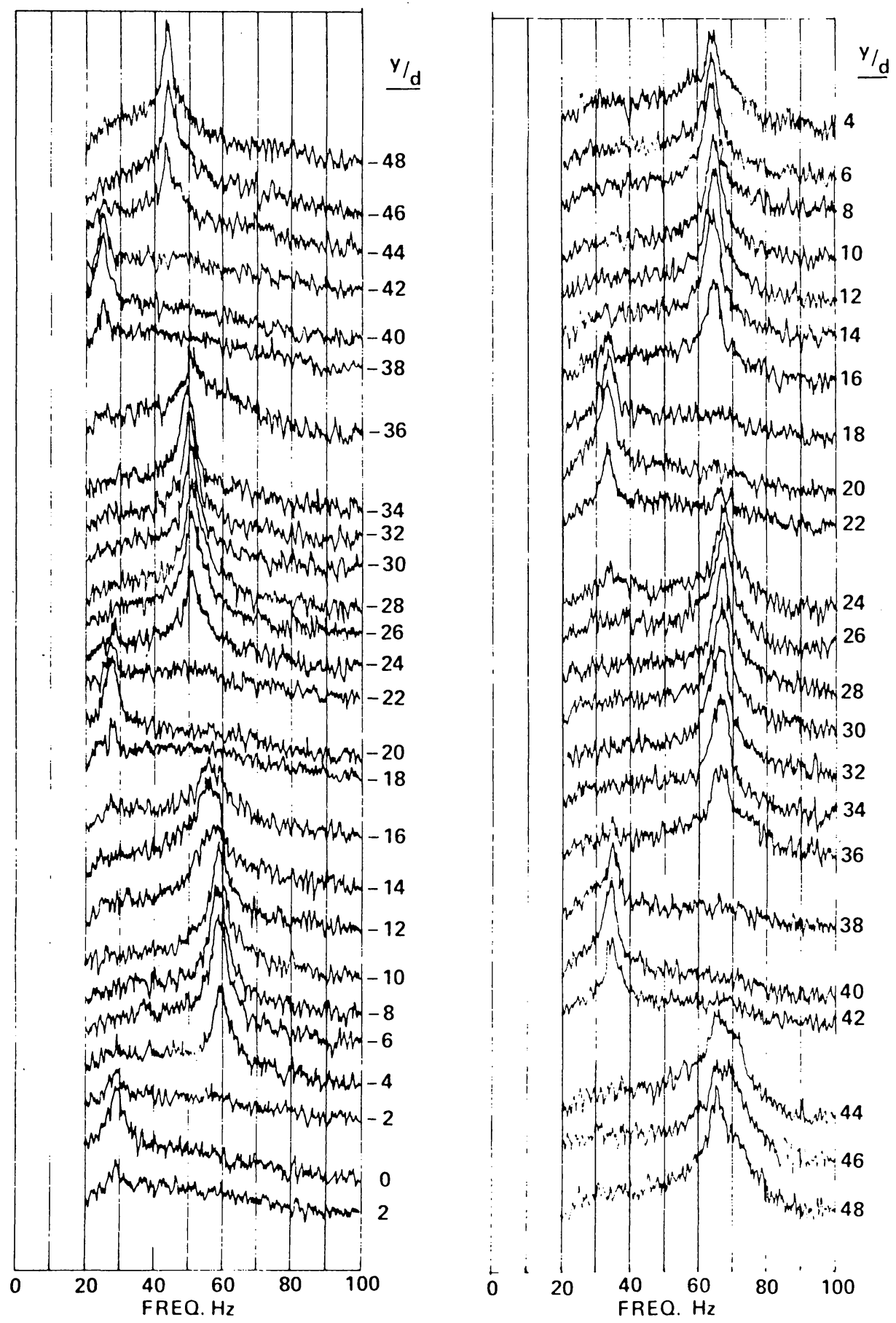

Figure 43. Power Spectra Showing the Spanise Variation of the Vortex Shedding Frequency, $\operatorname{Re}_{\mathrm{c}}=2.96 \mathrm{x}$ $10^{3}$, Five Spheres, No Vibration, $\beta=0.0053$. 


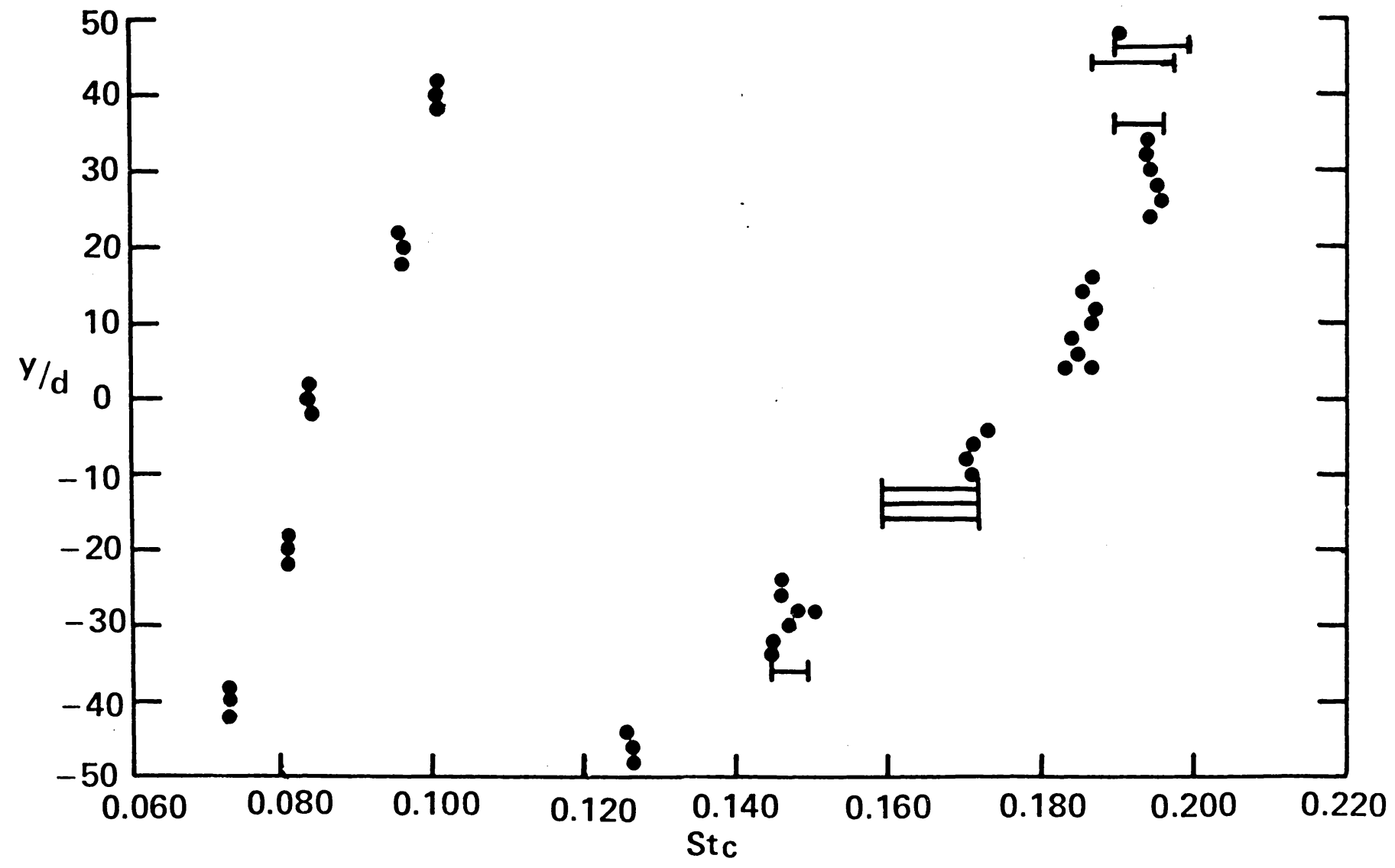

Figure 44. Spanwise Strouhal Number Variation, $\operatorname{Re}_{c}=2.96 \times 10^{3}$, Five Spheres, No Vibration, $B=0.0053$. 

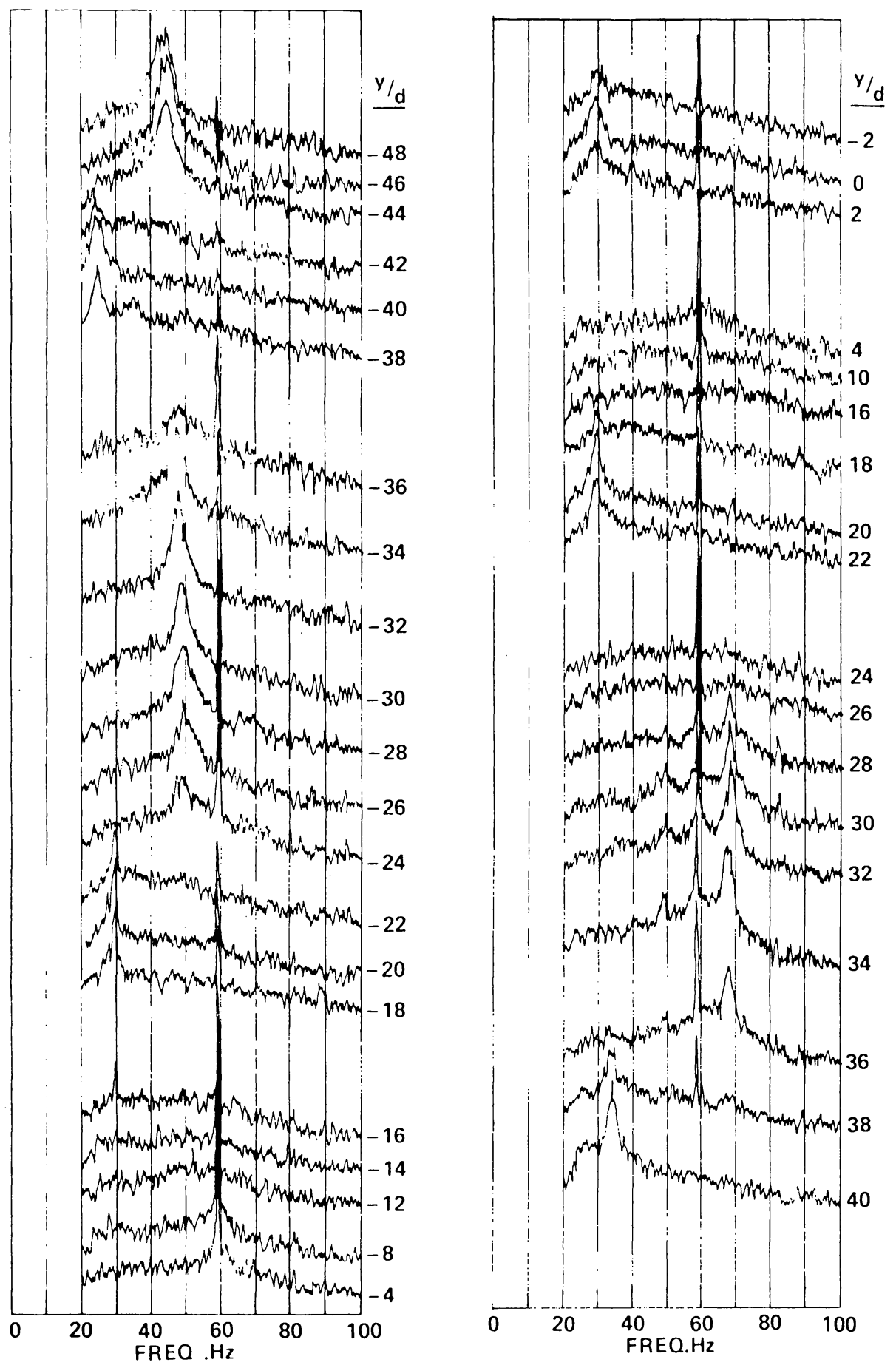

Figure 45. Power Spectra Showing the Spanwise Variation of the Vortex Shedding Frequency, $\operatorname{Re}_{c}=2.96 \mathrm{x}$ $10^{3}$, Five Spheres, First Mode Vibration, $a / d=$ $23.3 \%, \quad \beta=0.0053$. 


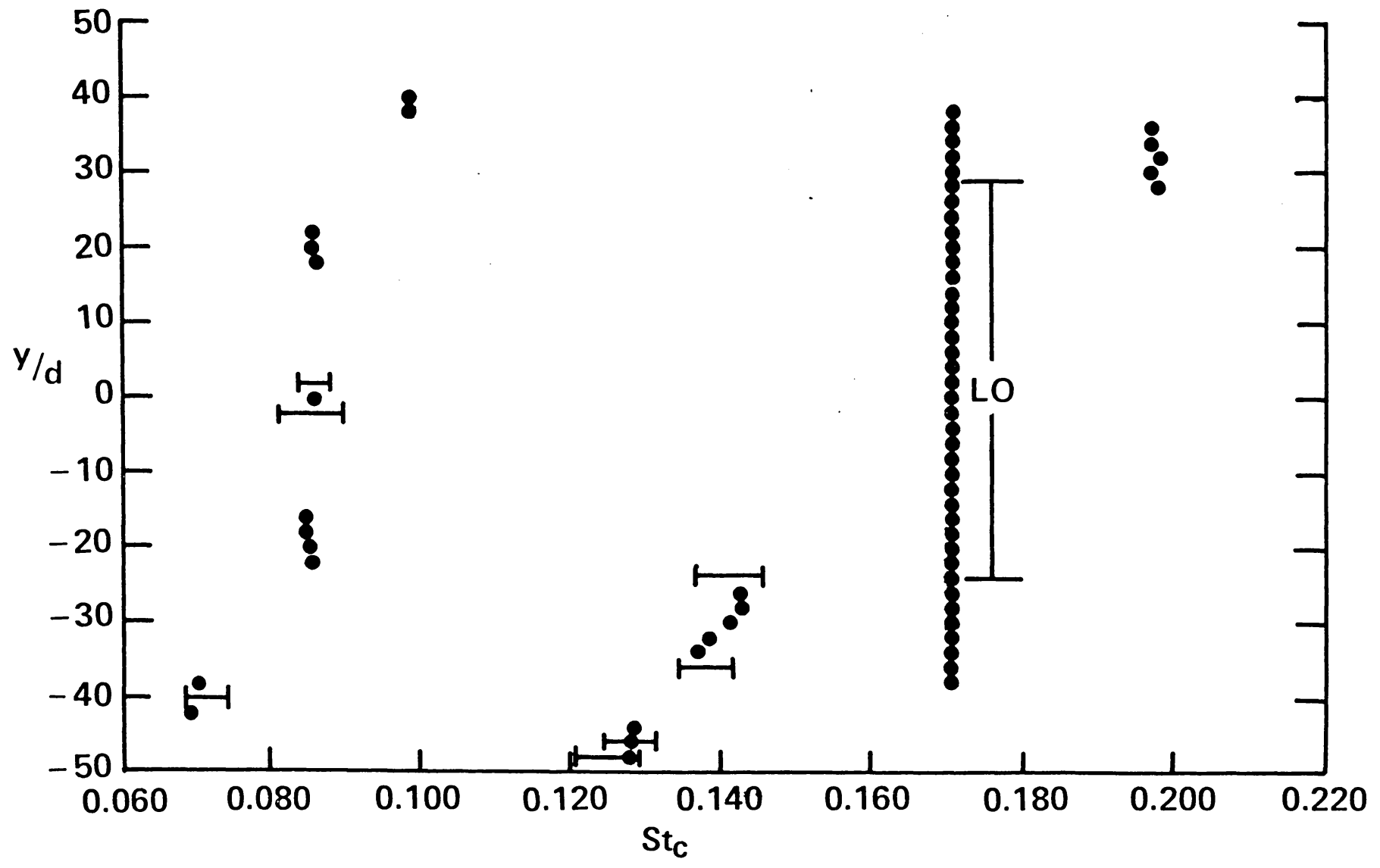

Figure 46. Spanwise St rauhal Number Variation, $\operatorname{Re}_{c}=2.96 \times 10^{3}$, Five Spheres, First Mode Vibration, $a / d=23.3 \%, \quad B=0.0053$. 

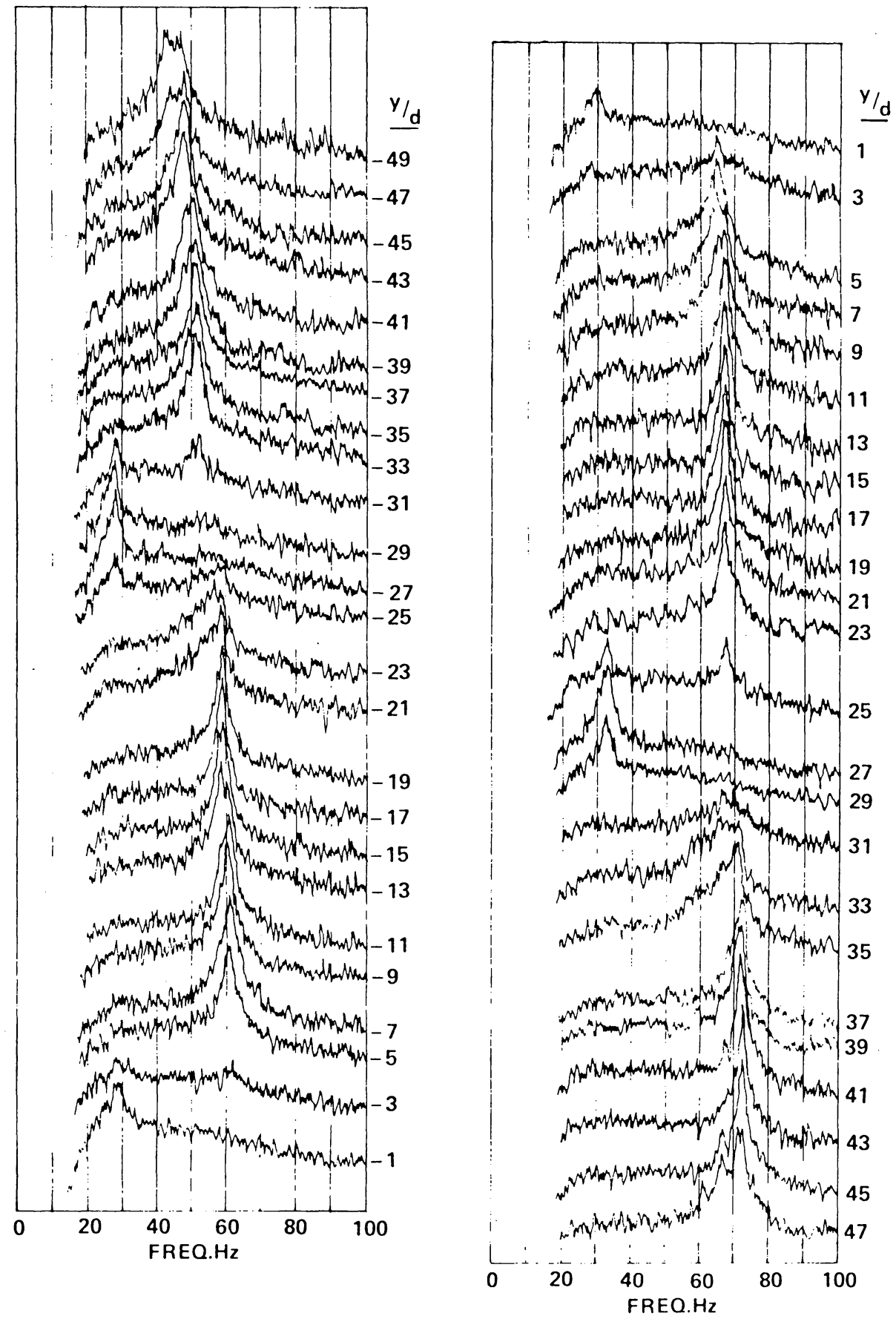

Figure 47. Power Spectra Showing the Spanwise Variation of the Vortex Shedding Frequency, $\operatorname{Re}_{c}=2.96 \mathrm{x}$ $10^{3}$, Three Spheres, No Vibration, $\beta=0.0053$. 


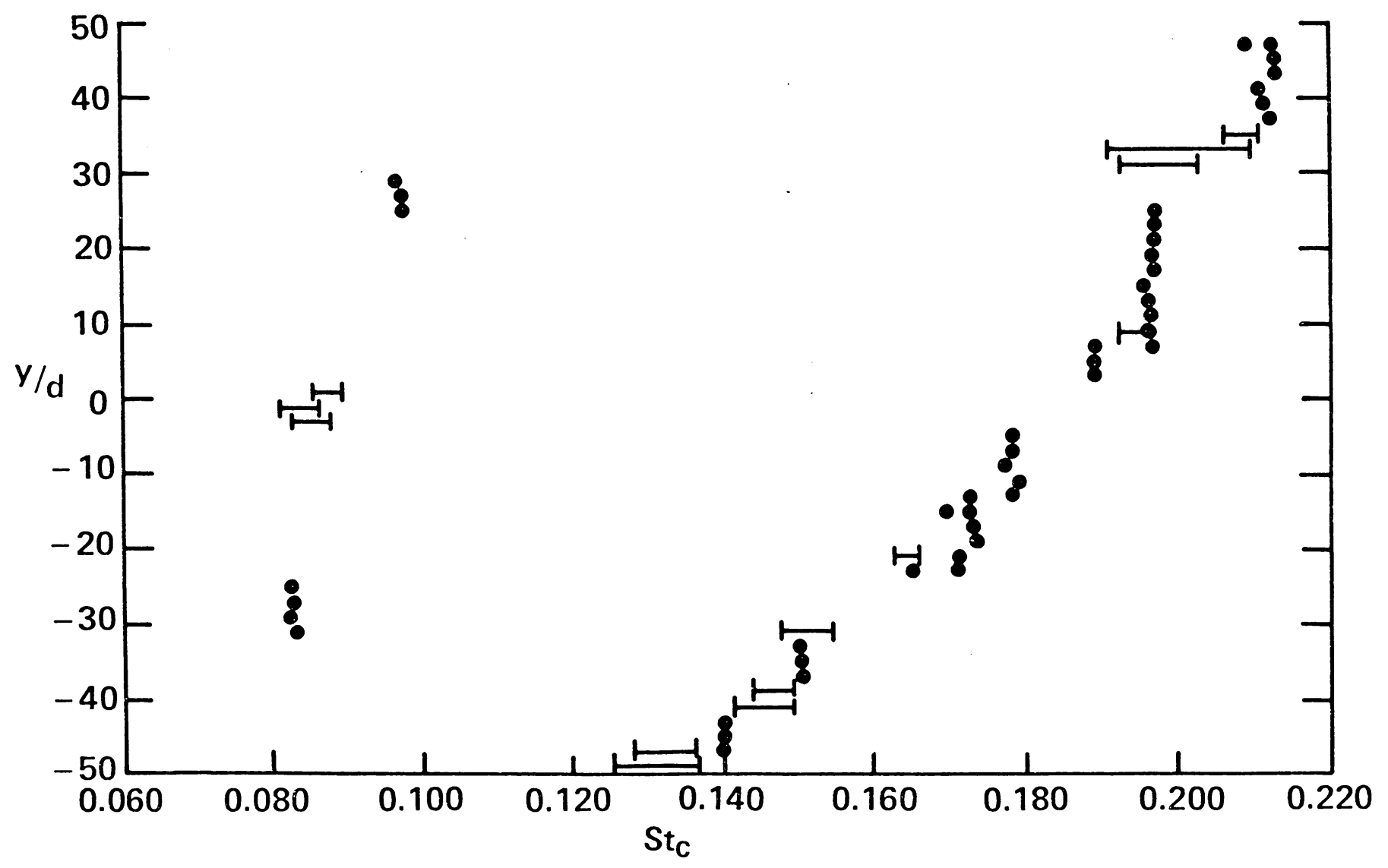

Figure 48. Spanwise St rouhal Number Variation, $\operatorname{Re}_{c}=2.96 \times 10^{3}$, Three Spheres, No Vibration, $\beta=0.0053$. 

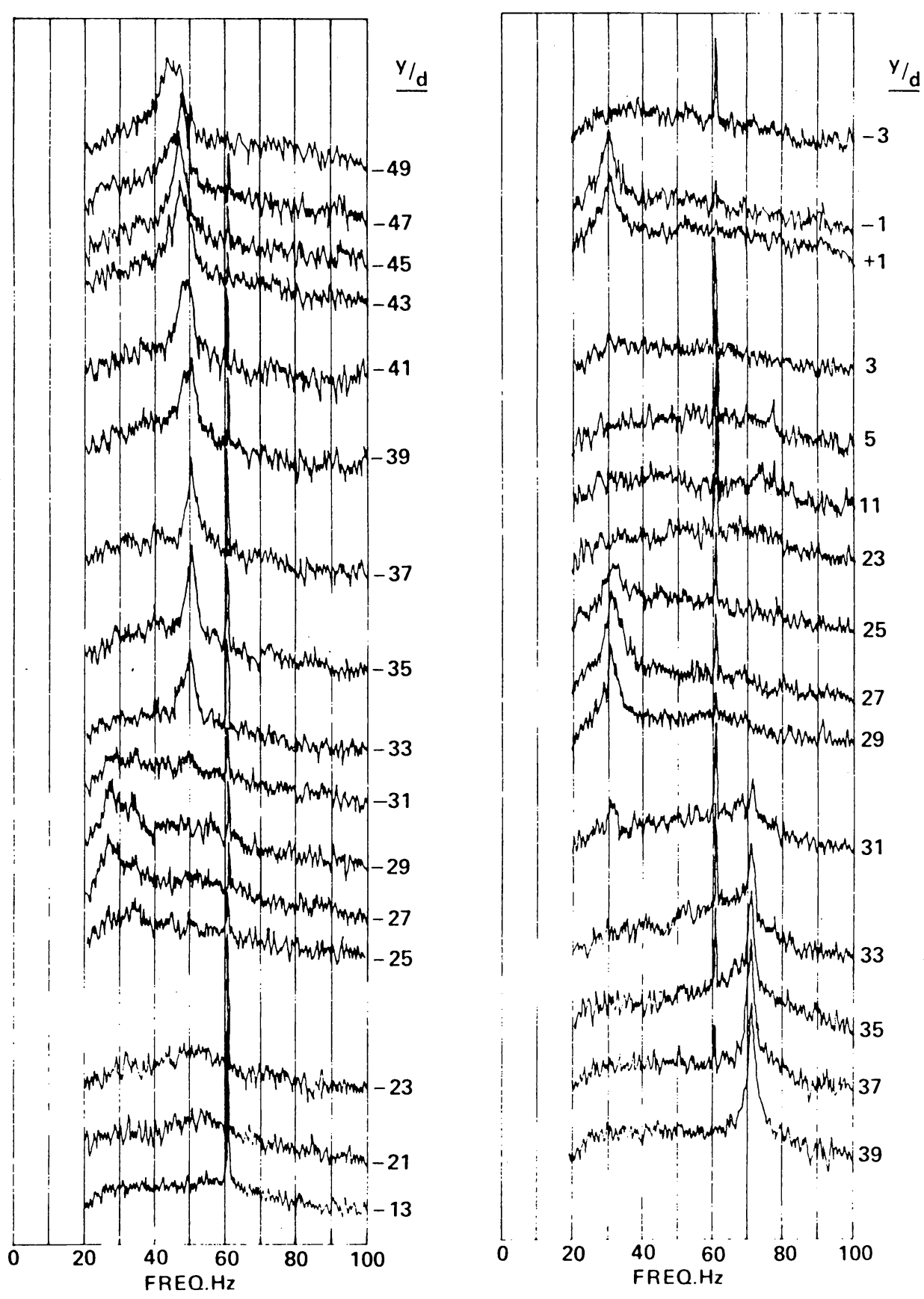

Figure 49. Power Spectra Showing the Spanwise Variation of the Vortex Shedding Frequency, $\operatorname{Re}_{c}=2.93 \mathrm{x}$ $10^{3}$, Three Spheres, First Mode Vibration, $a / d=$ $23.5 \%, \beta=0.0053$. 


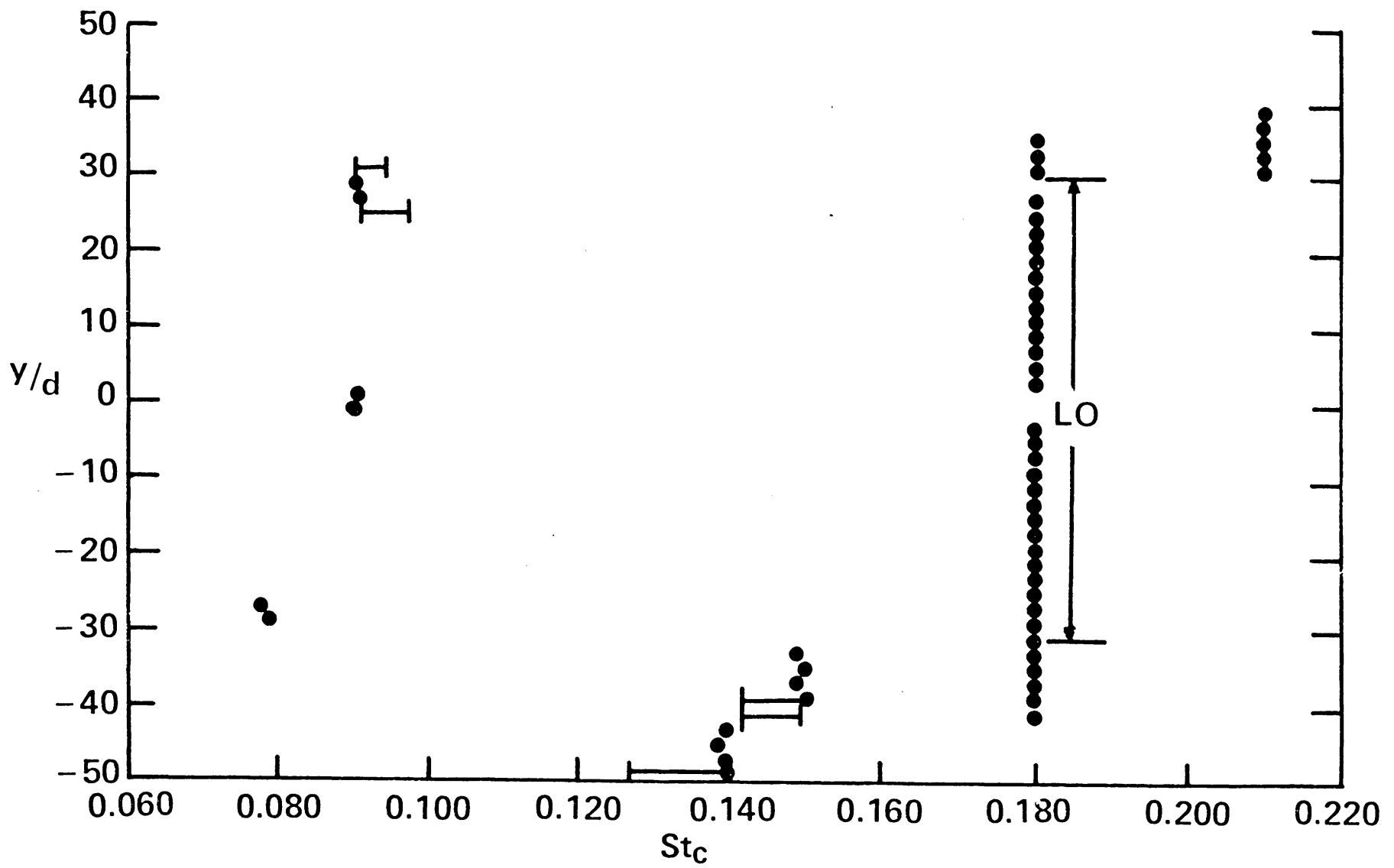

Figure 50. Spanwise St rouhal Number Variation, $\operatorname{Re}_{C}=2.93 \times 10^{3}$, Three Spheres, First Mode Vibration, $a / d=23.5 \%, \quad \beta=0.0053$. 
Appendix B

Previously Established Methods 
The experimental techniques that were employed in the present study to measure vortex shedding frequencies, vortex formation lengths, wake widths, wind tunnel velocity and turbulence values, vibration amplitudes and vibration frequencies had been developed and proven accurate in previous wind tunnel studies perfromed at VPI\&SU by the present author $[31,32,38]$. Some comments regarding the accuracy of these techniques are provided in this appendix to increase the readers confidence in the results that were presented in this report.

The spanwise velocity and turbulence profiles that were measured during the present tests showed no significant statistical variations from those measured previously [32]. Two hundred individual velocity or turbulence values were averaged to obtain each data point in the profiles. Both the uniform $(\beta=0.0)$ and linear shear $(\beta=0.0053)$ flows in the present study had turbulence intensities less than one percent across the spanise working distance. The flows were not considered to have been influenced by turbulence. Power spectra of the fluctuating hot wire anemometer signal revealed that there were no extraneous frequency components (wind tunnel blade frequencies, sixty cycle noise, wi re screen vortex shedding frequencies, etc.) present in the flow or measurement system. Variations in the kinematic viscosity, $v$, due to ambient temperature and pressure changes were taken into account when calculating the flow Reynolds number. Blockage effects created by the presence of the three-dimensional traverse, cable endplate supports and the measuring instruments in the freestream were insignificant. 
The Zonic FFT with a DMS 5003 computer was used to calculate power spectra of the fluctuating hot wire (cable vortex shedding frequency measurement) signals. The signals were band-pass filtered before they were input into the FFT to eliminate aliasing. The Strouhal numbers, calculated using the measured values of the cable vortex shedding frequencies, were all virtually equivalent throughout the subcritical flow range covered by the tests. These values were consistent with those measured previously for circular cylinders. Power spectra of the fluctuating hot wire signal in the freestream flow above the cable revealed that no extraneous frequencies were present. The measured values of the forced cable vibration frequencies were generally consistent with the calculated values of the ideal (no bending stiffness) natural vibration frequencies of the cable under the same conditions. When both the vortex shedding and forced cable vibration frequencies were simultaneously present in the fluctuating hot wire signal, no false addition and/or subtraction frequency comonents appeared in the power spectra of the signal.

There was great care taken to accurately measure the values of the vortex shedding frequencies in order to set the band-pass filter to the correct values prior to measuring the position of the vortex formation lengths in the Reynolds number range covered by these tests. The values of $\mathrm{L}_{\mathrm{f}}$, measured behind the circular cylinder were in general argreement with previous authors results. Low pass filtering was used to eliminate contamination of the wake width traces by higher frequency multiples of the vortex shedding frequency - The 
values of $\mathrm{L}_{\mathrm{w}}$ measured behind both the high aspect ratio cable and cylinder were virtually equivalent and nearly equal to previous authors results. When the streamise and vertical hot wire traverses were performed to measure $\mathrm{L}_{\mathrm{f}}$ and $\mathrm{L}_{\mathrm{w}}$ respectively, the traverse was operated at a low speed in order to allow three second averaging to be used in the rms meter to smooth out the signal trace on the strip chart recorder.

Al 1 the time based signals were monitored on an oscilloscope. This assured that no large extraneous noise signals were present or generated within the equipment, and also assured that all the equipment was functioning properly. 
The vita has been removed from the scanned document 
VORTEX SHEDDING FROM A VIBRATING CABLE

WITH ATTACHED SPHERICAL BODIES IN

A L INEAR SHEAR FL OW

by

Rodney Drew Peltzer

(ABSTRACT)

Marine cables of ten comprise an integral part of a larger structural system, such as an offshore drilling platform. They are also used to support marker buoys, as well as anchored or towed instrument arrays. Consequently, the resonant flow-induced oscillation of these cables, caused by vortex shedding, is extremely undesirable because of the associated damaging phenomena.

The present study, which employs hot wire anemometry as the principal investigative tool, was undertaken to examine the behavior in the near wake of a flexible, helically wound, high aspect ratio $(\mathrm{L} / \mathrm{d}=107)$ marine cable in a linear shear flow (steepness parameter $B=0.0053)$ at centerline Reynolds numbers between $2.0 \times 10^{3} \leq \operatorname{Re}_{\mathrm{c}} \leq 4.2 \times 10^{4}$.

Particular attention was paid to lock-on or "synchronization" related changes associated with uniform and sheared flow past the cable when it was forced to vibrate in the first mode. The study was extended to include an analysis of the effects on vortex shedding synchronization phenomena generated by placing spherical bluff bodies along the cable span. 
The frequency and reduced velocity boundaries of the lock-on regions, for both the cable and sphere-cable combination, as a function of vibration amplitude up to a/d $=0.322$, were measured, plotted and analyzed. The critical reduced velocity of the vibrating cable was $U_{r}^{*}=5.50$, and for the sphere-cable combination $U_{r}^{*}=3.39$. A sudden increase in the vortex formation length in the near wake of the vibrating cable occurred during perfect synchronization $\left(U_{r}^{*}=5.50\right)$. Shortly thereafter, the formation length returned to stationary cable values.

At $\operatorname{Re}_{c}=2.96 \times 10^{3}$, the spanwise vortex shedding frequencies behind the stationary and vibrating cable in a linear shear flow (with and without the presence of spherical bluff bodies) were measured, plotted and the results comared. A stable cellular vortex shedding frequency structure, strengthened by the synchronous vibration, existed along the span of the cable. The presence of and spacing between the spherical bodies along the cable span significantly affected the spanwise character of the near wake cellular structure. The length of the locked-on region was substantially increased (almost doubled) when the spherical bodies were present along the span of the cable. 